\title{
Abstracts XVII Congress of the Spanish Society of Gerodontology. Estepona, Spain, March 23 - 25 2017- Meeting Abstract
}




\section{- Oral Presentation 1}

TITLE:- Surgical management of leokoplakic lesions. A case study.

AUTHORS: Romero Gutiérrez C, Almena Martínez L, Leco Berrocal I, Martínez Rodríguez N, Gutiérrez Sánchez L, Fernández Cáliz F.

Hospital Virgen de La Paloma, Madrid.

Introduction: Leukoplakia is defined as "a white, attached, hyperkeratotic plaque which presents on a mucous surface and which cannot be characterized as any other condition". Its occurrence is higher among males and people above 40 years of age.

The present case relates to a 67-year-old male who presented for consultation after detecting a longstanding leukoplakic lesion in his gingiva.

The goal of this communication is to describe a clinical case of a male patient, analysing the clinical and surgical management of geriatric patients with leukoplakic lesions, as well as to assess the percentage of relapse from this condition.

Clinical Case: The 67-year-old patient was admitted to our Service with a referral from his general odontologist due to the presence of a recurring leukoplakic lesion located at the gingival level in absence of premolars and left maxillary molars.

The patient's medical background includes type II diabetes and hypertriglyceridemia.

The intraoral examination reveals an homogeneous whitish plaque measuring $1 \mathrm{~cm}$ by $1 \mathrm{~cm}$, which can't be removed by scraping.

An excisional biopsy of the lesion was performed, and an anatomopathological analysis was requested.

Conclusions: Leukoplakia is a relatively common entity wherein the odontologist must know the clinical manifestations, as well as the clinical-surgical management and the relapse percentage.

\section{- Oral Presentation 2}

TITLE:- Hemodynamic changes during dental treatments at normotensive and hypertensive geriatric patients.

AUTHORS: Olmo González B. Martín Gili D. Ribera Uribe $M$.

Universitat Internacional de Catalunya. Barcelona. Department of dentistry for Special Patients and Gerodontology.

Objectives: To evaluate the variation of blood pressure in normotensive and hypertensive patients with antihypertensive treatment, during surgical and non-surgical dental treatments. And with and without local anesthesia with vasoconstrictor.
Material and Methods: The study involved 100 normotensive patients/100 hypertensive patients with antihypertensive treatment. Each group was formed by $(n=50)$ with surgical treatment: $((n=25)$ anesthesia and vasoconstrictor vs $(\mathrm{n}=25)$ anesthesia without vasoconstrictor) and $(n=50)$ with non-surgical treatment: $((n=25)$ without anesthesia vs $(n=25) 20$ anesthesia and vasoconstrictor and 5 without vasoconstrictor). Blood pressure was evaluated by Electronic Sphygmomanometer OMRON M6 (HEM-7001-E) and Holter monitoring PM50 NIBP/SPO2 in 5 different time-points.

Results: There was an increase in Systolic Blood Pressure at the beginning of the surgical treatment with local anesthesia with vasoconstrictor, and finally decreased. In non-surgical treatment there was a decreased at the beginning and with vasoconstrictor remained stable.

The Diastolic Blood Pressure had no effect interaction compared to surgical and non-surgical treatments. With use of vasoconstrictor decreased initially, while without vasoconstrictor increased.

The Heart Rate decreased initially with surgical and non-surgical treatments, except in normotensive patients with non-surgical treatment which increased. After the treatment, the heart rate increased, except in normotensive and hypertensive patients with nonsurgical treatment. It was similar according to use of vasoconstrictor or not.

Conclusions: The dental treatments in normotensive vs hypertensive geriatric patients with antihypertensive drugs, imply a variation in the fluctuations of blood pressure during surgical and non-surgical dental treatments, and with and without local anesthesia with vasoconstrictor.

\section{- Oral Presentation 3}

TITLE:- Mandibular fixed rehabilitation by guided surgery and immediate loading in geriatric patients.

AUTHORS: Haidar Wehbe A, Pérez Hernández D, Ramos Palenzuela M, Matos Garrido N, Moreno Muñoz J, Velasco Ortega E.

Master of Implant Dentistry. Faculty of Dentistry. University of Seville.

Objectives: The introduction of new clinical implant protocols has promoted the realization of guided surgery in dental implant treatment. The aim of this study was to present clinical results of treatment with implants inserted by the technique of guided surgery and immediate loading in edentulous mandible.

Materials and Methods: Fully edentulous mandible patients were diagnosed by cone beam tomography and treated with 8-10 Galimplant $®$ implants for rehabilita- 
tion by the technique of guided surgery and immediate loading. Immediately after surgery a full resin provisional immediate restoration was placed. At 6 months, the final restoration was performed. The clinical followup period was at least 70 months of functional loading. Results: 20 patients were treated with 188 implants. The clinical findings demonstrate an excellent clinical response of this type of protocol, free of postoperative complications in patients undergoing surgery. From an implantologic point of view, the results indicate a success rate of $98.9 \%$. Two implants were lost during the healing period with the provisional prosthesis. After a mean functioning period of 80.6 months from the final restoration, there was no late complications.

Conclusions: This study indicates that treatment with dental implants by guided surgery and immediate loading in the mandible is a high success rate therapeutic alternative in geriatric patients.

\section{- Oral Presentation 4}

TITLE:-Short implants in atrophic posterior areas of the maxilla and mandible: current status.

\author{
AUTHORS: Merchan Morales S, Ruíz Sáenz PL, \\ Sanz Alonso J, Santos Marino J, Martín Ares M, \\ Martínez González JM. \\ Hospital Virgen de la Paloma. Master in Oral Surgery and Implan- \\ tology.
}

Objectives: To evaluate the success and survival of short implants placed in atrophic posterior areas of maxilla and mandible and compare it with the results of the longer conventional implants.

Material and Methods: We have done a literature review in PubMed database over 40 articles about the use of short implants made between 2011 and 2016. Inclusion criteria were the use of implants of length between 4 and $7 \mathrm{~mm}$. and follow up one year, at least, from the load.

Results: A total of 40 items on 65.006 implants placed in 29.014 patients were analyzed. The results were similar to those obtained with the use of longer implants, finding no statistically significant differences in complications and failures between the two procedures.

Conclusions: In elderly patients with atrophic posterior areas of the maxilla and mandible short implants can be a faster and less aggressive alternative than other techniques (regeneration and vertical bone augmentation, lateralization of dental nerve, etc.).

Short implants offer percentages of success and survival similar to those of the longer implants.

We must be aware of certain considerations and specificity in these implants regarding biomechanics, surgical technique and implant design.

\section{- Oral Presentation 5}

TITLE:- Immunological aspects of healthy periimplant tissue in submerged vesus no submerged implants.

AUTHORS: Martín Ares M, Merchán Morales S, Ruíz Sáenz PL, Sanz Alonso J, Santos Marino J, Martínez González JM.

Virgen de la Paloma Hospital. Madrid.

Objectives: To determine the characterization of the main lymphocyte subtypes present in the peri-implant mucosa, absent from the disease and to establish whether there are differences between mucosal and uncovered implants.

Material and Methods: A total of 31 patients who were treated with the unitary implants located in the right and left maxillary premolar an si of the AvantblastR Phibo TSA surface were included in this longitudinal study. Therefore, a total of 62 implants were studied, of which 31 were submerged and 31 were exposed with healing heads. Circular samples were taken at the head of the implants, while in the same situations in which the implants were found with the abutments of healing, a semicircular an sión was made at a vestibular level. Once the samples are collected, they are deposited in sterile containers with physiological serum and sender, within an interval of not more an two hours, to the Immunology Service of the Hospital Ramón y Cajal.

Results: The predominant lymphocyte subtypes in the oral mucosa have been, in decreasing order, CD4 +, CD8 + and NK. There have been statistically significant differences between immersed and no submerged implants, in relation to the CD8 + and NK lymphocyte subpopulations.

Conclusions: Non-immersed implants, potentially, present a greater susceptibility to infectious processes.

\section{- Oral Presentation 6}

TITLE:- Surgical difficulty and postoperative complications in the third molar extraction in patients older than 65 years.

AUTHORS: Sanz Alonso J, Santos Marino J, Martín Ares M, Merchán Morales S, Ruíz Sáenz PL, Martínez González JM.

Master of Oral Surgery and Advanced Oral Implantology, Hospital Virgen de la Paloma. Madrid.

Objectives: To evaluate if the age of the patients influences the surgical difficulty and the appearance of postoperative complications in the surgical third molar extraction in patients older than 65 years. 
Material and Methods: Prospective observational study of 200 patients divided into two groups (older than 65 years (G1) and between 45 and 65 years (G2)) of 100 individuals each. All of them underwent surgical extraction of a third retained lower third molar from which their position, position, number and root anatomy and relation with the dental nerve were previously recorded to establish their surgical difficulty. Data on pain, inflammation and need for analgesics were collected later in the postoperative period by means of a questionnaire provided to the patient.

Results: Both study groups obtained similar surgical difficulty values regarding their position, position, number and root anatomy. However, the onset of postoperative complications was greater in the G1 group in which patients needed twice as much analgesics and 1.5 times more pain than the G2 group. As for inflammation, this was greater in the G1 group, where $53 \%$ of the patients rated it as "important" at 48 hours of the intervention, compared to $21 \%$ of patients in the G2 group.

Conclusions: Old age is a risk factor for the development of postoperative complications, and therefore, it is recommended that the third molar extraction be performed at an earlier age.

\section{- Oral Presentation 7}

TITLE:- Relationship between the OHRQoL (Oral health related quality of life) and the HRQoL (Health related quality of life) in elderly people of Valencia and comparison with other countries.

AUTHORS: Sáez Prado B, Haya Fernández MC, Sanz García MT.

Universidad Cardenal Herrera CEU, Valencia.

Aim: The aim of this study was to investigate if there is a relationship between the OHRQoL and the HRQoL of elderly people aged 65 years or more in Valencia, Spain.

Material and Methods: We designed a cross-sectional survey with 2 quality-of-life instruments validated for use in Spain: the OHIP-14 (used to measure the quality of life related to the oral health) and the EURO-QoL 5D together with a visual analogue scale (VAS) used to measure the quality of life related to the general health. Subjects: 202 adults (103 men and 99 women). Age: 65 years of age and over. Setting: Randomly selected senior citizen's social clubs of Valencia.

A literature search was performed in Pubmed to know the state of play using the keywords: OHRQoL, HRQol, OHIP-14, EURO-QoL 5D, Not Implants.

Results: We found statistically significant relationship (p-value $<0.05$ ) between Euro-QoL5D and OHIP-14 as well as between the EuroQol-5d and the VAS. Several studies in different countries have found a link between the OHRQoL and the HRQoL through divers instruments of quality of life.

Conclusions: The oral quality of life is related to the general quality of life. By influencing in factors that benefit the oral quality of life, we can improve the general quality of life of the elderly.

\section{- Oral Presentation 8 \\ TITLE:- Atrophic maxilla: treatment with zy- gomatic implants.}

AUTHORS: Ordoñez Fernandez E, Caride Lamas AD, Sánchez Zorrilla A, García Sánchez AJ, Olivan Molina S, Fernández Domínguez $M$.

Máster en Cirugía Bucal e Implantologia. Universidad San Pablo CEU. Madrid.

Objectives: Severe reabsorption of the maxilla can make treatment difficult by conventional implants. The diverse alternatives need multiple surgeries, donor sites and a considerable prolonged time for consolidation and maturation. Zygomatic implants have been described as a therapeutical alternative to rehabilitate significant defects in the maxilla, frequently observed in geriatric patients.The objective is to review the published literature to evaluate the treatment success with zygomatic implants in patients atrophic posterior maxilla.

Material and Methods: A search in PubMed database was made, looking up articles between 2006 to 2016, in which were reported geriatric patients with atrophic posterior maxillary treated with zygomatic implants, in which success has been observed with a minimum of 12 months of follow-up

Results: Three different surgical techniques has been described to place zygomatic implants: the classic sinus window technique, the sinus slot technique and extra sinus zygomatic implants.The most common type of rehabilitation was the placement of fixed prosthesis, with loading implants after 3-6 months or immediate loading.The average success rate was over $90 \%$ and the most frequent complication was maxillary sinusitis. The overall level of patient satisfaction was high.

Conclusions: Zygomatic implants have a high success rate and are a suitable alternative to treat severe atrophy in the posterior maxilla. 


\section{- Oral Presentation 9}

TITLE:- Glandular odontogenic cyst in the geriatric patient. Clinical case report.

\section{AUTHORS: Coello de la Cruz L, Gala Penagos E, Santos Marino J, Rubio Alonso L, Buesa Bárez JM, Martínez González JM. \\ Hospital Virgen de la Paloma. Master in Oral Surgery and Implan- tology.}

Introducción: The glandular odontogenic cyst is an unusual lesion whose behavior is more aggressive than other odontogenic cysts. It's presented in a wide range of ages and it has no clinical or radiographic features that allow us to differentiate it from other intraosseous cysts.It can be distinguished by histopathology. It shows a squamous stratified epithelium with a glandular aspect.

Clincal Case: A 66-year-old male came to our practice asking for tooth extractions and prosthetic rehabilitation upon implants. He is asymptomatic, without pathological findings in the extra-oral examination, and intra-orally he presents several remaining root canals in maxillary and mandibular.A panoramic X-ray was requested to proceed with the extraction of radicular remains, observing a radiolucent lesion located in the right anterior area of the maxilla. It's delimited, has a regular contour, and a size of 3 by $3,5 \mathrm{~cm}$. A TC was requested to correct surgical planning. Then proceed to perform the extraction of the radicular remains, a total excision of the lesion, and it was sent for a histopathological study.The results reported the diagnosis of a glandular odontogenic cyst that had been removed completely with injury-free surgical margins and surrounding healthy bone tissue.The last accomplished TC noted an evolution of favorable ossification of the area.

Conclusion: The glandular odontogenic cyst has a high recurrent power, so it's imperative to do a radiographic study as a part of the follow-up study, even when surgery has been meticulous.

\section{- Oral Presentation 10}

TITLE:- Fracture of the sinus floor in a geriatric patient.

AUTHORS: Arnedo Martínez AM, Martínez González JM, Santos Marino J, Rubio Alonso L, Buesa Bárez JM, Barbi Actis J.

Master in Oral Surgery and Implantology Virgen de la Paloma de Madrid.

Introduction: Fracture of the sinus floor represents a relatively frequent complication in the field of Implantology and Oral Surgery. Within the latter, tooth extrac- tions constitute the most important etiological factor, causing invasion of the sinus cavity with an immediate inflammatory response of the antral mucosa or a orosinus fistula.The purpose of this paper is to present the clinical consequences and therapeutic management of this type of fracture.

Clinical Case: A 67-year-old male patient who came to the Emergency Department because of intense pain and sensation of fluid passing through the nose, after tooth extraction of 27.A intraoral examination, the presence of a sutured alveolus with an aspect of the normal covering mucosa is observed. The Vansalva maneuver was positive, a panoramic radiograph and a Waters Projection were requested, observing a sinus occupation and radiodense image compatible with the radicular rest. After the informed consent, it was proceeded by anthrostomy, the removal of the hyperplastic mucosa and of the radicular rest, and the wound was closed with a tight seal.

Conclusions: The radiographic study before the dental extractions, helps to prevent the displacement of radical remains in the maxillary sinus.

\section{- Oral Presentation 11}

TITLE:- Oral manifestations secondary to pharmacological treatment of rheumatoid arthritis. Review of the literature and case report.

AUTHORS: Peña Cardelles JF, Cano Durán JA, Ortega Concepción D, Zarrias C, García Rodríguez MD, Hernández Vallejo G.

Título Propio de Especialista en Medicina Oral. Universidad Complutense de Madrid.

Objective: To show the oral manifestations whose etiology is related with the pharmacological treatment of rheumatoid arthritis (AR), reviewing the most recent literature.

Material and Methods: A search of scientific articles of the last 5 years is carried out in the Pubmed database, using keywords "Rheumatoid arthritis", "Oral manifestations" and "Drugs".

Results: The treatment of AR is based mainly on pharmacological therapy, which is responsible for manifestations at the oral cavity. We report the case of a 65 -year-old woman with AR under treatment with methotrexate and tocilizumab, who came to the clinic with a 1 month ulcer on the lateral border of the tongue. It is relatively frequent the appearance of ulcers in the oral cavity by drugs used in the treatment of AR, such as gold salts and methotrexate. Others such as penicillamine, azathioprine, and sulfasalazine may lead to petechiae or gingivorrhages secondary to thrombocytopenia. Peni-cillamine may cause taste alterations, and chloroquine hyperpigmentations. 
Immunosuppressants may lead the develope of mycotic infections, oral ulcers, and in the case of cyclosporine, gingival hyperplasia. Also, the use of several drugs can develop hyposialia and xerostomia.

Conclusions: The oral manifestations of AR derive mainly from pharmacological therapy, which must be known for the correct diagnosis and treatment of the oral pathology of these patients.

\section{- Oral Presentation 12}

TITLE:- Oral lesions in geriatric patients.

\section{AUTHORS: Benítez Rodríguez M., Pérez Belmonte P., Hernández Montero S., Cobo Yera S., Lozano Bustamante $\mathbf{F}$. \\ Master Universitario de Implantología y prótesis implantosopor- tada. Universidad ALFONSO X EL SABIO.}

Objetives: Study oral pathologies to know health conditions and the possibility of treatment. In this project we'll evaluate the presence of oral mucosal injuries in patients over 65 years old through an examination and a correct medical history.

Material and Methods: Study a representative sample of patients over 65 years old at ALFONSO X EL SABIO (Madrid) Clinic, comparing the total number of characters and the presence of oral injuries according to follow-up. The injuries will be diagnosed according to criteria established by the W.H.O. (World Health Organization).

Results: Prevalence of lesions and more frequent lesion, as well as prevalence according to sex.

Conclusions: Implement prevention programs as well as adequately dealing with established cases.

\section{- Oral Presentation 13}

TITLE:- Implants loading protocols in patients older than 65 years of age.

\section{AUTHORS: Cobo S, Lozano F, Benítez M, Pérez P , Herzandez $\mathbf{S}$. \\ Master Universitario de Implantología Oral y prótesis Implantoso- portada. Universidad Alfonso X el Sabio.}

Introduction: The continuous growth of the population 65 years of age and older indicates that the incidence rate of complete edentulism will remain constant or even increase over coming decades.

As the average life expectancy is constantly increasing, and with that the percentage of the population aged 65 and older, it becomes clear the need for prosthodontic treatment will increase. Although removable dentures are an option, the oral function and comfort frequently are not favorable. Nowadays, our patients demand fixed rehabilitations with dental implants. However, the extended healing time prior to implant loading associated with the conventional loading protocol is a disadvantage from the patient perspective. Therefore, there is a tendency in the field of oral implantology tor reduce treatment time and simplify procedure in order to increase patient acceptance and satisfaction with favorable result.

Clinical Case: To report on the effect of immediate implant loading with fixed prostheses compared to early and conventional loading on implant and prosthesis survival, failure, and complications in a patient 65 years of age.

Conclusions: With careful patient selection and using implants with rough surfaces, immediate loading with fixed prostheses in edentulous patients has the same effect on implant survival, failure, and complications as with early and conventional loading in maxillary and mandibular arches.

\section{- Oral Presentation 14 \\ TITLE:- Guided surgery. Advantages and dis- advantages against conventional oral surgery.}

AUTHORS: Aguilar Martín C, Pérez Corral I, Ordoñez Fernández E, Cabezas Mojón J, Fernández Domínguez $\mathbf{M}$.

Master in oral surgery and implantology.CEU San Pablo University. Madrid.

Objetives: Analize the benefits that guide surgery brings against conventional surgery and the complications that have been detected to avoid or solve them during the procedure.

Material and Methods: It has been done a bibliographic research of articles between 2012-2015 using "COEM" "Pubmed" data bases and key words "guided surgery"'fully edentulous patients".

Results: Guided surgery allows implant placement in a place that is planned previously so that a accurate forecast of the protest result is obtained. Despite of flapless surgery has disadvantages like the lack of assesment of soft tissue and the lack of visualization of the operative field there is evidence that this technique decreases swallow and postoperative pain so that analgesics taking, wich benefits specially geriatric and compromised patients.The intraoperative complications commonly detected have been the break of the surgery guide and the lack of adaptation of the prosthesis.

Conclusions: Guided surgery allows obtain a forecast of the final results especially in edentulous patients where there are not anatomical references. For its realization it is necesary to know the setbacks that can occur and the solutions. 


\section{- Oral Presentation 15}

TITLE:- New in-vitro methodology to compare microfiltration, resistance to fatigue and torsion in different implant connections.

\author{
AUTHORS: Martín Gili D. Punset Fusté M. Giner \\ Tarrida Ll. Olmo González B. Ribera Uribe M. Gil \\ Mur FJ. \\ Universitat Internacional de Catalunya. Barcelona. Department of \\ dentistry for Special Patients and Gerodontology.
}

Objetives: To establish a novel in vitro experimental protocol to quantify the microfiltration of implant abutment interface in different implant designs under dynamic load conditions.

Material and Methods: Two different designs of implant systems were tested, using 20 samples of external connection / 20 samples of internal connection. One subgroup $(\mathrm{n}=5)$ was used to observe and evaluate the microgap before and after load cycling. A scanning electron microscope (Neon40 with GEMINIcolumn) was used for the implant-abutment microgap observation. An other subgroup $(n=15)$ was used to evaluate the microfiltration dynamics of loading for each connection. Mechanical tests were performed using a universal testing machine tension-compression-torsion fatigue model MTS Bionix 370 (MTS-USA). A compressive force of $700 \mathrm{~N}$ and torsion of $3^{\circ}$ was applied for a total of 100.000 cycles at $2 \mathrm{~Hz}$ of load frequency and submerged at $37 \mathrm{C}^{\circ}$. Parameters to simulate human mastication and according to the specifications detailed in ISO 14801 standards.

Results: The internal connection implants presented lower microgaps and microfiltration than the external ones. An increase of the microgap and microfiltration were observed in all samples as the number of cycles increased.

Conclusions: Treatment with implants in the elderly patient represents an alternative to maintain a favorable oral health. Therefore, this new methodology for the quantitative evaluation of microfiltration implant in vitro cyclic load using a dynamic model to evaluate objectively the microfiltration under load conditions, allows to assess the type of implant better to the characteristics of the elderly patient.

\section{- Oral Presentation 16}

TITLE:- Dental implants and oral cancer: a review.

AUTHORS: Roca Millan E, Domínguez Mínger J, Sabater Recolons MM, Jané Salas E, López López J.

Master of Oral Medicine, Oral Surgery and Oral Implantology. Department of Odontostomatology, Faculty of Medicine and Health Sciences (Dentistry). University of Barcelona.
Aim: Analyse the published cases in the literature of patients with dental implants who have developed cancer in its close proximity and observe which malignant lesions may be associated.

Material and Methods: Bibliographic search in Pubmed/Medline of clinical cases and case series published in the last 15 years using the keywords: dental implant AND cancer.

Results: A total of 24 studies were revised obtaining a sample of 47 patients, 28 women and 19 men. The average age resulted in 66 years, belonging a $40 \%$ to the seventh decade. In the $85 \%$ of the cases, cancer had developed in the jaw. More than $82 \%$ had risk factors like tobacco, alcohol, poor oral hygiene, the presence of leukoplakia, erytroplakia or oral lichen planus, or previous radiation in the oral cavity. The $53 \%$ of the cases had previously suffered from cancer. The most common clinical presentation was an exophytic mass, followed by ulceration and pain. In more than $25 \%$ of the patients the malignancy mimicked peri-implantitis. Diagnosis was OSCC in $87 \%$ of the cases, followed by metastasis.

Conclusions: Despite not being a high prevalent issue, its consequences are severe. So in the presence of periimplant lesions, especially in patients with risk factors or who had previously suffered from cancer, it should be considered a malignant lesion as a possible diagnosis.

\section{- Oral Presentation 17 \\ TITLE:- Peripheral granuloma of giant cells. A case report.}

AUTHORS: Ruiz de Carlos C, Martínez Puga M, Santos Marino J, Rubio Alonso L, Barona Dorado C, Martínez González JM.

Máster cirugía bucal el implantología. Hospital Virgen de la Paloma, Madrid.

Introduction: Peripheral Granuloma of giant cells is a begignant Tumour-related injury with chronic inflammatory caracteristics apearing at gingival level. It may present implication of the bone and periodontal tissues. It is the most common giant cells injury of the jaws. It results in response to a local irritation or to a chronic trauma.

Clinical Case: 67 year old male patient has been remitted to our service presenting a painless lump that produce labial displacement.

Clinical examination shows a localized lump in premaxilla of $2,5 \times 2 \mathrm{~cm}$ with soft consistency that covers the teeths 22,21 and 11 in a thrid coronal level. It presents sligthly congestive colour at the top and movable. The rest of the oral cavity examination is considered within the normal parameters. 
The patient is informed about the posible diagnosis of benignant lump, asking for his consent in order to remove it and its histopathologic study.

The procedure is performed under local anesthesia, making a Newman flap with mucoperiostial sweeping of the premaxilla región, removing the lump injury, proceding to replace the mucoperiostial flap and finally suturing the wound.

Amoxicilina 750mg v.o./8h; Diclofenaco sódico 50mg v.o./8h. is prescribed as a post operative treatment.

After a week from the medical intervention and after a postoperative period with no problems we proceeded to remove the stitches. The patient was informed about the definive diagnosis of the giant cells granuloma.

Conclusions: When it comes to a tumour-related injury it is fundamental to perform a complete surgical exeresis and make a histopathologic diagnosis in order to obtain a propper final diagnosis.

\section{- Oral Presentation 18}

TITLE:- Zidovudine as pharmacological treatment in parotid lesions.

AUTHORS: Hurtado Celotti D, de la Calle Cañadas C, Martínez Rodríguez N, Gutiérrez Sánchez L, Fernández Cáliz F, Barona Dorado C.

Master Cirugía Bucal e Implantología. Hospital Virgen de la Paloma de Madrid.

Introduction: Zidovudine is an antiretroviral drug which is indicated and has demonstrated to be effective in the treatment of Human Inmunodeficiency Virus infection. It has recently been proposed as an alternative against several parotid lesions related to this virus as well as Epstein Barr Virus.

Case Report: 60-years-old female patient presenting swelling in the right parotid area, appearing to be welldefined, slightly depressible and soft, from 5 years ago. CT and MRI were performed and both showed right parotid (22 x $34 \mathrm{~mm})$ and left $(14 \times 28 \mathrm{~mm})$ enlarged. A fine needle aspiration was performed and the presence of aciner and ductal cells in plaques with no other nodal components was observed. Serological tests highlighted positive presence of antibodies to cytomegalovirus. "Bilateral benign lymphoepithelial lesion" was established as diagnosis based on clinical features and observed findings. The pharmacological approach was chosen using zidovudine v.o. and 6 months later a favorable but moderate response with slight decrease of both tumors was observed.

Conclusions: Within the pharmacological or surgical therapeutic possibilities, zidovudine represents a non-invasive alternative whose main adverse effect is a state of pancytopenia, and therefore requires periodic controls.

\section{- Oral Presentation 19}

TITLE:- Follicular excision of a dentigerous cyst associated with impacted lower third molar with preservation of the inferior alveolar nerve and placement of the osteosynthesis plate. A case report.

AUTHORS: Santos Marino J, Martin Ares M, Merchán Morales S, Ruiz Sáenz PL, Sanz Alonso J, Martínez González JM. Orofacial Surgery Service Hospital Virgen de la Paloma.

Introduction: The dentigerous cyst is the second most common odontogenic cyst. The symptoms in the Dentigerous Cyst are scarce and rarely reaches large proportions. When this happens is detected clinically, because it produces bone expansion and facial asymmetry due to the excessive internal pressure of the lesion, which predisposes to the production of pathological fractures by erosion of cortical bone, however, in most cases it is detected as a radiographic finding. We must also be attentive to observe if there is loss of teeth, intense root reabsorption of the adjacent teeth and pain, which are consequences of the continued enlargement of the cyst. On the other hand, is potentially capable of becoming an injury aggressive.

Case Report: We report a clinical case of a female patient of 65 years of age who had swelling in the affected area. After the pertinent radiological tests extraction was performed of the cystic lesion along with the third molar causing and proceeded to the placement of the osteosynthesis plate before the obvious risk of bone fracture.

Conclusions: All tooth should be considered a potential cyst by what turn prescriptive its evolutionary analysis and removal.

\section{- Oral Presentation 20}

TITLE:- Oral health-related quality of life in elderly people with mandibular 2-mini-implants overdenture.

AUTHORS: Haya Fernández MC, Cabo Pastor MB, Sanz García MT, Sánchez Solís EJ, Jover Cerveró A.

Universidad Cardenal Herrera Valencia.

Introduction: The conventional rehabilitation of the total edentulous lower patients presents great difficulties related to support, retention and stability of the mucosal prostheses. For this reason, rehabilitation with mandibular overdenture over 2 implants seems to be a more effective treatment option for older patients with large bone mandibular atrophies.Oral health-related quality 
of life has been studied mainly in the elderly, probably due to the general interest in this age group, which has shown an accelerated growth in the last decades in most countries.

Objectives: To evaluate the oral quality of life in elderly patients who are rehabilitated with overdentures on 2 mandibular mini-implants.

Material and Methods: Fifteen elderly patients aged 60-89 years with mandibular overdentures on 2 miniimplants (mini SKY bredent medical) have been rehabilitated. You have passed 2 oral quality of life questionnaires, Oral Health Impact Profile (OHIP-14). Geriatric Oral Health Index or GOHAI before and after prosthetic rehabilitation.

Results: Most of the treated patients are women who have for many years had conventional prostheses with many difficulties such as lack of stability, constant decubitus ulcers...

In relation to the oral quality of life after prosthetic rebalancing, the patients fundamentally improve, in the dimensions of physical pain and physical incapacity of the OHIP-14.

Conclusion: Mandibular overdentures on 2 implants improve the quality of life of edentulous patients.

\section{- Oral Presentation 21}

TITLE:- Exodontic teeth retained indications in the geriatric patient. A case report.

\section{AUTHORS: Marro Amador P, Franco Alfonso P, Leco Berrocal I, Martínez Rodríguez N, Gutiérrez Sánchez L, Martínez González JM. \\ Hospital Virgen de la Paloma.}

Introduction: Old age by itself does not contraindicate a surgical intervention, being that the quality of the technique should not change according to the age of the patient. Even so, geriatric patients should be carried out with a more detailed control before and after the intervention due to pluripathology, the lower capacity to respond to operative stress and polypharmacy.

Clinical Case: A 68 year old female patient shows a second and a third mandibular molar retained (37 and 38). In the radiological study an intimate relation between molars and the inferior dental nerve is observed. The intervention is performed with the usual technique for surgical extraction of retained third molars. The usual medical prescription was made in this case and the ususal higiene standards for the postoperative period. The patient presented half hemilabial parestesia. Vitamin complexes were prescribed and rutinary controls were carried out at 1, 3 and 6 months, noticing how the ansesthetized zone was decreasing. In the 1 year reviez after the surgery, the sensibility recovery is total.
Conclusions: It is essential the previus study of the old age patient for avoiding posible complications caused by the amount of pathologies that can suffer or the high number of medicines with wich they are treated. The surgical thechnique should always be the same regardless patient's age.

\section{- Oral Presentation 22}

TITLE:- Treatment of epulis fissuratum. A literature review.

AUTHORS: Maidagan Valderrama A, Romero Gutiérrez C, Leco Berrocal I, Martínez Rodríguez N, Gutiérrez Sánchez L, Fernández Cáliz F.

Máster en Cirugía Bucal e Implantología. Hospital Virgen de la Paloma. Madrid.

Aims: The aim of this review is to recognize the different treatments of the Epulis fissuratum and to check which is the most suitable.

Material and Methods:A literature review has been made in the database MedLine although PubMed during the years 2000 and 2017, using the key words: "Epulis fissuratum", "Inflammatory fibrous hiperplasia", "treatment, "dental laser".

Results: Epulis fissuratum is a hyperplastic growth of the mucosa which is the most prevalent oral lesion and is most frequenty located in the vestibular groove. Its diagnosis is made according to its clinical characteristics, and after its extraction, through a study of pathological anatomy. Epulis treatment may be non-surgical by removal or relief of the prosthesis, or surgical by removing the épulis using cold scalpel, electrical scapel, laser or cryogenization. The recurrence rate of epulis is $8,5-9 \%$.

Conclusions: Removal of epulis of the oral cavity can be treated by differents tecniques. The surgical laser is the first option due to intra and postoperative properties.

\section{- Oral Presentation 23}

TITLE:- Treatment with dental implants in geriatric patients with diabetes 2 .

AUTHORS: Salgado Peralvo O, Arenas Baldrich R, Cabanillas Balsera D, Jiménez Guerra A, Ortiz García I, Velasco Ortega E.

Master of Implant Dentistry. Faculty of Dentistry. University of Seville.

Introduction and Objectives: Today, implant dentistry constitute a therapeutic modality in the prosthodontic treatment of totally edentulous patients. This study 
reports the comparative evaluation of treatment with overdentures in the mandible in edentulous older patients with and without diabetes.

Material and Methods: 54 edentulous patients, 27 diabetic patients type 2 and 27 healthy patients, were treated with 108 Galimplant ${ }^{\circledR}$ implants for prosthodontic rehabilitation with overdentures in the mandible. Two implants were inserted in each patient. Implants were loaded after a healing free-loading period between 6 weeks with locator attaches. Clinical findings (implants and prosthodontics) were followed during at least 60 months.

Results: Two implants failed. One implant was lost during the healing period in each group. Clinical results indicate a survival and success rate of implants of $98.2 \%$ after a mean follow-up of 78.6 months. 54 overdentures were realized over two implants. Technical complications were related with plastic components of attaches and reported in 8 implants, 3 implants in diabetic patients $(5.5 \%)$ and 5 implants $(9.2 \%)$ in healthy patients. Mean marginal bone loss was $0.420 \mathrm{~mm}$ in diabetic patients $0.400 \mathrm{~mm}$ in healthy patients.

Conclusions: Clinical results of this study indicate that there are not differences in treatment with dental implants in geriatric patients with diabetes compared with healthy geriatric patients.

\section{- Oral Presentation 24}

TITLE:- Long-term clinical outcomes of early loading of mandibular overdentures in geriatric patients.

AUTHORS: Garcia Sanchez A, Gfrorer Reina A, Minaya Tueros H, España Lopez A, Jiménez Guerra A, Velasco Ortega E.

Master of Implant Dentistry. Faculty of Dentistry. University of Seville.

Introduction: Today, oral implantology constitute a therapeutic modality in the prosthodontic treatment of patients with total tooth loss. This study reports the evaluation of edentulous patients treated with overdentures in the mandible by early loading of titanium implants with acid-etched surface.

Material and Methods: 13 edentulous patients were treated with 45 TSA Defcon ${ }^{\circledR}$ acid-etched surface implants for prosthodontic rehabilitation with overdentures in the mandible. All implants were inserted in onestage. Implants were loaded after a healing free-loading period between 6 weeks. Clinical findings (implant and prosthodontics) were followed during 15 years.

Results: Clinical results indicate a survival and success rate of implants of $94,4 \%$. One implant were lost during the healing period. Late complications (after early loading) were reported, two implants were lost by periimplantitis. $76.9 \%$ of patients were treated with overdentures with bar $(61.5 \%$ by 4 implants and $15.4 \%$ by 3 implants), and the rest of patients (23.1\%) with overdentures retained with 2 implants.

Conclusions: Clinical results of this study indicate that prosthodontic rehabilitation of edentulous patients with mandibular overdentures supported by acid etched-surface titanium implants can achieve osseointegration and were loaded early as a successful dental treatment.

\section{- Oral Presentation 25 \\ TITLE:- Maxillary implant-supported fixed re- habilitation with submerged implants in geria- tric edentulous patients.}

\author{
AUTHORS: Cabanillas Balsera D, Escuín Jiménez \\ P, España Guerrero E, Jiménez Guerra A, Matos \\ Garrido N, Velasco Ortega E. \\ Master of Implant Dentistry. Faculty of Dentistry. University of \\ Seville.
}

Introduction and Objectives: Fixed oral rehabilitation represents a form of implant treatment in patients with a long period of fully edentulous. The aim of the study was to show the evaluation of fixed rehabilitation on implants in geriatric patients with edentulous maxilla.

Material and Methods: 24 maxillary edentulous patients were treated with fixed implant restorations with 210 submerged, external connection, sandblasted and acid etched implants. Implants were loaded after a healing period of 8 weeks.

Results: 24 fixed cemented rehabilitations were performed. The mean clinical follow-up was 70.4 months. The mean marginal bone loss were $0.80 \mathrm{~mm}$ (range: $0.40-2.40 \mathrm{~mm}$ ). Complications occurred in 7 patients. 5 implants (2.4\%) presented periimplantitis. 3 implants $(1.4 \%)$ were lost, consequently the success rate was $98.6 \%$.

Conclusions: The clinical findings of this study indicate that fixed oral rehabilitation on implants in the maxilla have a high success rate in geriatric patients.

\section{- Oral Presentation 26}

TITLE:- Implant treatments in geriatric patients with oral lichen planus. A case report.

AUTHORS: De la Calle Cañadas C, Jalil Abumalham D, Buesa Bárez JM, Barona Dorado C, Fernández Cáliz F, Martínez González JM

Máster de Cirugía Bucal e Implantología Hospital Virgen de la Paloma. 
Introduction: Oral lichen planus is a common benign inflammatory disease affecting mainly middle-aged and elderly people, with a prevalence of approximately $0.2-2 \%$. This disease is more common in females than in males and it has most often been reported in middleaged patients 30-60 years of age. It tends to follow a more chronic course and is often accompanied by acute exacerbations.

Purpose: The aim of this study is to emphasize the importance of the maintenance in patients rehabilitated with implants with oral lichen planus.

Case Report: A 67-year-old female patient came to the Oral Surgery Service of Virgen de la Paloma Hospital (Madrid) and presented with ulceration of few days duration. The medical history of the patient was unremarkable. The intraoral examination showed an ulcerated lesion in the bucal mucosa. The patient has been rehabilitated previously with implants. She refers pain in the ulcerated region and surrounding the implants. Furthermore, she has been rehabilitated with implants previously. The diagnosis was oral lichen planus. Topical corticosteroids were administrated. At the follow-up visit generated a substantial clinical improvement and the patient was free from subjective symptoms.

Conclusions: It is vital the maintenance in oral lichen planus patients with implant rehabilitations. Good hygiene control reduces exacerbations.

\section{- Oral Presentation 27}

TITLE:- Premilinary study of knowledges and concerns of the dental profession about Gerodontology.

\author{
AUTHORS: Franco Alfonso P, Candita C, Leco Be- \\ rrocal I, Rubio Alonso L, Santos Marino J, Barona \\ Dorado C. \\ Máster de Cirugía Bucal e Implantología Hospital Virgen de La \\ Paloma (Madrid).
}

Objectives: The aim of the present study is to describe the knowledge that dentists have on Gerodontology in order to treat patients over 65 years of age. Due to the rising numbers of geriatric population in Spain demand to prepare dentists on the capability to offer to this group of patients a specific attention according to their skills and needs.

Material and Methods:An online survey was handed to dentists and fifth-grade dental students to identify skills and concerns they may find regarding geriatric population. The survey consisted of 15 items which covered from the type of training they received, alongside issues and concerns of professionals at the time of treating a geriatric patient, to the resources used to solve those problems.
Results: Most of those who answered the survey $(n=124)$ did not have any specific education $(45,2 \%)$, yet they considered that it would have been necessary as they highlighted the obvious difference between the treatment of adult and geriatric patients $(58,1 \%)$. Furthermore, an $85,5 \%$ was concerned about drug therapies of the geriatric patients, amongst which biphosponates and cardiotonics stand out. In opinion of the surveyed, a $46,8 \%$ see the necessity of a regulated education at least as modular courses upon that subject area.

Conclusions: $43 \%$ of the respondents consider that their knowledge on Gerodontology is rather limited, which reveals the necessity of promoting the training of treatment of geriatric patients.

\section{- Oral Presentation 28}

TITLE:- Use of glass ionomers reinforced with resin before radicular caries in senior adult associated with Sjörgren's Syndrome: rev. bibliographic.

\section{AUTHORS: Moradas Estrada M 1. Álvarez López B} 2. Villa Vigil M A. 3 .

IProfesor del Servicio de Odontología Conservadora. Universidad de Oviedo. 2 Práctica privada en Tapia de Casariego. 3 Catedrático de Odontología Conservadora y Materiales Dentales. Universidad de Oviedo.

Introduction: Morphological deterioration, with neck and third root exposure, as well as cognitive impairment that also affects the quality of brushing, favors the appearance of root caries in the elderly adult population. Essential is a long-term preventive treatment, since the aetiology of dryness or Syndrome of Sjörgren will continue present.

Aim:

1. Show the relationship of root caries associated with dry mouth.

2. To quantify the mechanical improvement of the IV in the cervical third.

3. Show better polish and final aesthetics.

4 . To determine a lower recurrence of caries in the medium term. (5 years)

Material and Methods: A systematic review of the evidence was carried out in the last 6 years, in the main indexed journals. The result was 196 articles, which after applying the different in / exclusion criteria, were reduced to 45 . Statistical analysis was performed.

Results: Necessary is the use of adhesive materials of the same color of the tooth, although the IV lack a sufficient mechanical resistance and worse esthetic. The IV hybrids have been created, which results in materials with better mechanical, aesthetic properties

Conclusions: With the obvious limitations of this study, we affirm: 
1 . The use of IV with resin, reduced by $65 \%$ the appearance of new caries.

2. The aesthetic capacity was similar to that of the resins.

3. Mechanical capacity compared to composite resins was not statistically worse.

\section{- Oral Presentation 29}

TITLE:- Survival of narrow-diameter dental implants.

AUTHORS: Plejo Rojas R. Uribe Flores J. Gil Manich V. Olmo González B. Ribera Uribe M.

Universitat Internacional de Catalunya. Barcelona. Department of Special Patients and Gerontology.

Aim: Analyze the survival of narrow-diameter dental implants based on osseointegration.

Material and Methods: A bibliographic review was carried out in the PubMed database of articles published between the years 2000-2016.

The keywords used were dental implants, small diameter, osseointegration, narrow implant.

Results: According to the reviewed literature, the survival rates collected in the reviewed studies for narrow diameter implants were as follows: 1.8 diameter with $100 \%$ survival, 2.2 diameter with $100 \%$ survival, 2.4 diameter with $100 \%$ survival, 2.5 Diameter with survival of $98 \%, 3.0$ diameter with survival from $95.5 \%$ to $100 \%, 3.25$ diameter with survival of $93.8 \%, 3.3$ diameter with survival from $92.3 \%$ to $100 \%, 3.4$ diameter with survival of $94 \%$ at $99 \%, 3.5$ diameter with survival from $93 \%$ to $100 \%$.

Conclusions: Implants of narrow diameter have a high survival in the studies found. The factors that were used to determine the survival of the narrow-diameter implant were bone loss and controls performed in periods of 12 months to 10 years.

\section{- Oral Presentation 30}

TITLE:- The role of leucocyte-rich platelet-rich fibrin (L-PRF) in the treatment of osteonecrosis of the jaws (ONJ).

AUTHORS: Cano Durán JA, Ortega Concepción D, Peña Cardelles JF, García Riart Monzón M, Paredes Rodríguez VM, Hernández Vallejo G.

ONJ is defined as an area of necrotic bone that has a difficult spontaneous healing.

Objectives: Show a different therapeutic alternative in ONJ. For that, a reviewing of the most recent literature has been done,
Material and Methods: To make this work, a literature search was made on PubMed database using the following keywords: "Osteonecrosis", "Jaws", "L-PRF" y "Platelet-rich fibrin". The inclusion criteria were: articles published in English or Spanish in the last 6 years. Results: New methods for the treatment of this lesion are currently being studied. Among these methods we find the use of lasers, hyperbaric oxygen, ozone therapy, stem cell immunotherapy, teriparatide administration and L-PRF membranes.

The L-PRF clot contains platelets and leukocytes. This clot gives rise to a fibrin matrix that releases growth factors and proteins, which help to heal wounds. It is a simple and economical method compared to other therapeutic alternatives.

In 2014 these membranes were started to use in patients with ONJ after intravenous bisphosphonate therapy. 30 days after treatment, no evidence of bone exposure was found. These results seem hopeful. Thus, more studies are being carried out to prove the true efficacy.

Conclusions: Use of L-PRF is an effective technique to accelerate natural healing of tissues. It's obtained from the patient's own blood, avoiding a possible immune rejection. Therefore, we consider that it's a viable therapeutic alternative in this type of lesions.

\section{- Oral Presentation 31 \\ TITLE:- Orofacial repercusion of temporal vas- culitis: Clinical case.}

AUTHORS: Puente Fernández S, Celotti D, Martínez Rodríguez N, Gutiérrez Sánchez L, Fernández Cáliz F, Barona Dorado C.

Máster de Cirugía Oral e Implantología. Hospital Virgen de la Paloma de Madrid.

Introduction: Temporal arteritis is the most common form of vasculitis. It is more common in women and Nordic countries. The incidence increases with age, with an average appearance at age 71. It is an inflammation of medium and large arteries, with a preference for temporal, ophthalmic, posterior ciliary and vertebral arteries. Its etiology is unknown and the diagnosis is based on clinical symptomatology and biopsy of the artery (sensitivity varies $15-40 \%$ ). Its treatment includes corticosteroids, biological therapy and immunosuppressants.

Objectives: The objective of this study is to know the clinical picture characteristic of this disease and the precautions to be taken into account during dental treatment.

Clinical Case: A 67-year-old female patient attending the Oral Surgery and Implantology Service at Virgen de la Paloma Hospital. The clinical history revealed 
the existence of intermittent temporal headache with mandibular and occipital irradiation. Temporary artery biopsies were requested with negative results but the diagnosis was maintained after a good response to corticosteroid therapy.

Conclusions: Temporal vasculitis should be suspected in the presence of mandibular claudication, temporal headache, sensitivity to the touch of the scalp, visual alterations and / or rheumatic polyneuropathy. Special care should be taken with side effects and drug interactions.

\section{- Oral Presentation 32}

TITLE:- Medical emergencies in the dental office. A literature review.

AUTHORS: Melero Alarcón C, Montañes de la Fuente A, Solis Gonzalez S, García - Riart Monzón M, Paredes Rodríguez V.M, Hernández Vallejo G.

Postgraduate in Oral Medicine. Complutense University. Madrid.

Introduction: The dental treatment of geriatric patients is becoming more frequent. The group of elderly patients is in increasing expansion, which implies greater possibilities of presenting a possible medical urgency. Despite their low frequency, they must be identified and treated quickly and effectively. Vasovagal syncope is the most frequent, followed by emergencies secondary to ischemic heart disease, hypoglycemic, epileptic, anaphylactic, asthmatic, and airway obstruction. The different situations will be described as well as their treatment.

Objectives: To review the incidence of medical emergencies in the dental field, to accurately describe the diagnosis, monitoring and treatment recommended for the dentist.

Material and Methods: A literature review was carried out in English and Spanish in Pubmed / MEDLINE and Discovery Ebsco databases with the keywords: Medical emergencies in the dental practice.

Results: We classified the different clinical situations into several groups:

1. Vasovagal syncope

2. Hypertensive emergencies: acute myocardial infarction, angina pectoris

3. Asthmatic emergencies and airway obstruction

4. Hypoglycemic crisis

5. Epileptic crisis

6. Anaphylaxis

7. Stroke

Conclussions:

1. The incidence of medical emergencies in the dental office is infrequent. The most relevant is vasovagal syncope.
2. It is necessary for the dentist to keep in mind the medical situation of the patient, to know how to anticipate to the possible complications the patient could have and to apply the necessary measures in case there is an emergency in a dental office.

\section{- Oral Presentation 33}

TITLE:- Prevalence of oral lesions in a sample of elderly patients.

AUTHORS: Schiavo Di Flaviano,V; Domingo M; González Navarro B; Jané Salas E; Marí Roig A; López López J.

Master of Oral Medicine, Oral Surgery and Oral Implantology. Department of Odontostomatology, Faculty of Medicine and Health Sciences (Dentistry). University of Barcelona.

Introduction: According to the Population Data of 2016 of the National Institute of Statistics (INE), 24.46\% of the habitants in Spain are older than 60 years. With the increase of the life expectancy we face a greater exposure to pathogens, which added to the histological changes that occur in the epithelium by aging, results in a greater susceptibility to the development of oral lesions. With our study we intend to find which are the most prevalent oral lesions in elderly patients and their location.

Material and Methods: An analysis was made of the clinical data of biopsies performed between November 2011 and January 2017 in patients over 60 years old who attended the service of Medicine, Surgery and Oral Implantology at the Odontological Hospital University of Barcelona. The data collected

were: age, sex, location, clinical and histopathological diagnosis of the lesions. The lesions were distributed into three groups: benign, malignant and potentially malignant lesions.

Results: The most prevalent were benign lesions, followed by premalignant lesions, mainly lichen planus and leukoplakia, and in less number oral squamous cell carcinoma. In addition, bullous lesions such as pemphigus were also diagnosed.

Conclusions: Oral lesions are frequent in the elderly, its necessary to improve prevention, diagnosis and treatment systems, with special emphasis on the early detection of oral cancer and the follow-up of potentially malignant lesions.

\section{- Oral Presentation 34}

TITLE:- Stylocarotid syndrome in geriatric patient. A case report. 
AUTHORS: Candita V, Maidagan Valderrama A, Leco Berrocal I, Rubio Alonso L, Santos Marino J, Barona Dorado C.

Master en Cirugia Oral e Implantología, Hospital Virgen de la Paloma. Madrid.

Introduction: The symptomatic elongation of the styloid process is known as Eagle's Syndrome.

This pathology presents two variables: the "Classic syndrome" that is shown after a tonsillectomy or a trauma and the "stylocarotid syndrome" which is produced by the compression of the internal and external corotid arteries.

Clinical Case: 65-year-old woman was referred for pain assessment in the left retromolar trigone, bilateral mastoid and temporal region. At 25 years old, she was diagnosed of migraines, presenting dizziness with sporadic loss of consciousness and neck pain. The patient is currently under symptomatic treatment with zolmitriptan $2.5 \mathrm{mg}$, tetrazepam $50 \mathrm{mg}$ and NSAIDs. There's no surgical history, blood analysis shows only slightly elevated C-reactive protein.

The intraoral exploration evidenced: periodontitis, absence of caries and tumors, implantoprotesic rehabilitation in first and second quadrant and rehabilitation with fixed prosthesis in third quadrant. ATM has no pathology.

The orthopantomography shows a great bilateral elongation of both styloid processes. A diagnostic infiltration in both tonsils of $1 \mathrm{ml}$ of lidocaine $2 \%$ was realized, obtaining the complete remission of the symptoms. Magnetic resonance imaging (MRI) with gadolinium contrast was requested, which revealed the relationship of the styloid processes with the external and internal carotid arteries; confirming the diagnosis of carotidinia. The patient refused surgical treatment.

Conclusion: Dentists may be the first professionals revealing diagnose of stylocaroid syndrome.

\section{- Oral Presentation 35}

TITLE:- Prevalence of oral lesions in diabetes mellitus patients.

AUTHORS: González Serrano J, Sáez Alcaide LM, Paredes Rodríguez VM, López Pintor Muñoz RM, de Arriba de la Fuente L, López Quiles Martínez J, Hernández Vallejo G.

Departamento de Medicina y Cirugía Buco-Facial. Universidad Complutense de Madrid.

Objectives: About $26 \%$ of the Spanish population between 65 and 74 years suffer from diabetes mellitus (DM). There is no systematic knowledge about the prevalence of oral mucosal lesions in patients with DM compared to healthy people. The objectives of this study were: (1) to compare the prevalence of oral lesions in patients with DM compared to a healthy control group and (2) to know the most frequent lesions in DM patients compared to healthy population.

Material and Methods: The articles selected were: (1) original articles from scientific journals, (2) cross-sectional studies written in English or Spanish, (3) assessing the prevalence of oral lesions in patients with DM, (4) comparing the results with a healthy control group and (5) specifying the diagnosed oral lesions in patients with DM and the healthy population.

Results: The included studies showed a prevalence of $45-88 \%$ oral lesions in patients with type 2 DM compared to $38.3-45 \%$ in healthy individuals, and $44.7 \%$ in patients with type $1 \mathrm{DM}$ compared to $25 \%$ in the healthy group. Methodological quality was assessed using The Joanna Briggs Institute Prevalence Critical Appraisal Tool, which showed the low quality of existing studies. Conclusions: There is statistically significant prevalence of oral lesions in patients with type 1 and type 2 DM compared to a healthy population, being tongue alterations and prosthetic stomatitis the most frequent.

\section{- Oral Presentation 36}

TITLE:- Orthokeratinized Odontogenic cysts vs. Keratocystic Odontogenic Tumor, surgical finding: a clinical case.

AUTHORS: Farnés Montpeyó M, Schiavone Mussano R, Cuscó Albors S, Vidal Bel A, Marí Roig A, López López J.

Máster en Medicina, Cirugía e Implantología Oral. Facultad de Medicina y Ciencias de la Salud, Odontología. Universidad de Barcelona.

Introduction: A 65 years old female patient with no allergies, with medical record of bilateral deep hearing loss and chest cystectomy is referred to our service to extract the tooth 2.8 .

Clinical Case: During the extraction, we observed a white exudate appearing in large quantities in a "coagulated milk" form. We collected it and we sent it to the anatomophatologic service. Anatomophatologic result: keratin scales. We requested a CT scan and we did a second intervention where we extirpate the lesion. Anatomophatologic result: inflamed keratocystic odontogenic tumor.

Conclusions: It is important to analyze carefully the orthopantomographs. Do not close using the buccal fat pad if we are not sure if there are remains of lesion. Always analyse the suspicious material. 


\section{- Oral Presentation 37}

\section{TITLE:- The importance of prevention in insti-} tutionalized elders.

\author{
AUTHORS: Luelmo Reverte O. Isern Schaefer C. \\ Olmo González B. Gil Manich V. Ribera Uribe M. \\ Universitat Internacional de Catalunya. Barcelona. Department of \\ Special Patients and Gerontology.
}

Goals: Establish a preventive protocol in institutionalized elderly, through the use of Duraphat $\AA^{\circledR}$.

Material and Methods: A bibliographic review was performed in the PubMed database between the years 2000 and 2016 using the keywords: fluor, fluoride varnish, caries, root caries, institutionalized, oral health, prevention.

A preventive protocol of action with application of Duraphat ${ }^{\circledR}$ was established in 20 institutionalized patients of the Foundation Albà during the period September 2016-March 2017. Patients presented a minimum of one tooth per quadrant with a code 2 of categorization and description of root caries ICDAS. Duraphat $\AA$ was applied per quadrants, previously, plaque was removed, the area dried, and relative insulation. Controls were performed every month. The assessment of the state and activity of the lesions was done according to the ICDAS criteria.

Results: The group of teeth that presented a code 2 in categorization and description of root caries ICDAS, showed a stabilization of the caries (moderate).

Conclusions: The application of preventive dental protocols with Duraphat $\AA$ in the institutionalized patients, are effective, and allow to stabilize the state of the code 2 lesions that do not present a cavitation greater than $2 \mathrm{~mm}$. The lack of oral health attention of some institutionalized elderly people, promotes the necessity of addressing this population group problem. It would be necessary and recommendable to implement a preventive oral health program that includes preventive cares and dental training for health professionals, caregivers and family members of the patient.

\section{- Oral Presentation 38}

TITLE:- Use of diode laser in the treatment of injuries in oral soft tissues.

AUTHORS: Ortega Concepción D, Cano Durán JA, Peña Cardelles JF, Sánchez Garrido I, de Arriba de la Fuente L, Hernández Vallejo G.

Postgraduade in Oral Medicine, Faculty of Dentistry of the Complutense University, Madrid.

Objectives: To evaluate the efficacy and predictability of diode laser as a treatment of soft tissue injuries compared to other surgical methods in geriatric patients.
Material and Methods: A review of the most recent literature was carried out in the PubMed database, using the keywords "diode laser", "soft tissue", "oral cavity" and "surgery oral". Clinical trials, human studies, studies measuring clinical variables, systematic reviews and meta-analyzes, published between 2007 and 2017 were used as inclusion criteria.

Results: The review of the literature shows that the diode laser is a minimally invasive technology that offers great advantages, superior to those of the conventional scalpel, such as reduction of bleeding, inflammation and a lower probability of scars. Its effectiveness is comparable to that of other types of lasers, in addition to being an option of lower cost and greater ease of use. Its application in the soft tissues has been evaluated, proving to be a safe and effective method for the excision of lesions like fibromas, epulis fissuratum and the accomplishment of frenulectomies.

Conclusions: The diode laser can be used with very good results for the removal of lesions in soft tissues, being employed in small exophytic lesions due to their easy application, adequate coagulation, no need to suture and the slightest inflammation and pain.

\section{- Oral Presentation 39 \\ TITLE:- Sedation in the geriatric patient.}

AUTHORS: Almena Martínez L, Caño Gómez N, Leco Berrocal I, Martínez Rodríguez N, Gutiérrez Sánchez L, Fernández Cáliz F.

Hospital Virgen de la Paloma. Madrid.

Objectives: To know the different types of sedation that exist and their application in the geriatric patient, in addition to establishing the importance of the choice and management of these drugs in function of the type of pathology existing, individualizing each case.

Material and Methods: After a search of the past 20 years in some databases such as MEDLINE and CROCHANE, 18 articles were chosen applying the following inclusión criteria: language, year of publication, abstract contents.

Within geriatric patients there exist certain individuals who can benefit from sedation techniques such as those who present physical and/or psychic disability or any other disorder in their mobility.Two types of sedation are distinguished: superficial and deep.

According to the route of administration, they will be classified in inhalation, oral, rectal or intravenous. Depending on the type and duration of the sedation, a drug can be used instead of another; nitrous oxide and benzodiazepines: diazepam, lorazepam, alprazolam, midazolam and triazolam are the most used nowadays. For intravenous sedation, ketamine and propofol are the drugs of choice. 
The main pathologies to take into account, because of their frequency, in the individualization of each case are: arthritis, arterial hypertension, cardiovascular disease, respiratory and renal disease, and neurological and hepatic alterations.

Conclusions: Sedation offers a vast number of advantages when it comes to treat geriatric patients, however, special care must be taken and cases must be individualized since they are usually polymedicated.

\section{- Oral Presentation 40}

TITLE:- Management of obstructive sleep apnea in adults.

AUTHORS: Montañés de la Fuente A, Solís González S, Melero Alarcón C, de Arriba de la Fuente L, Serrano Valle J, Hernández Vallejo G.

Postgraduate in Oral Medicine, Faculty of Dentistry, Complutense University of Madrid.

Objectives: Obstructive sleep apnea (OSA) is a common disorder in the adult population, characterized by repetitive episodes of cessation of respiration due to collapse of the upper respiratory tract. OSA causes important symptoms, such as daytime somnolence, and it is associated with a significant morbidity and cardiovascular mortality. Different therapeutic possibilities have been proposed, and a literature review has been carried out to evaluate the indications, efficacy and role in clinical practice of each treatment option available for the management of OSA, as well as its possible adverse effects.

Material and Methods: A computer-assisted literature search was performed in the PUBMED/MEDLINE database using the key words "mandibular advancement devices " and "obstructive sleep apnea".

Results: Management of OSA includes changes in patient behaviour, Positive Airway Pressure (CPAP), oral devices and surgery. Its choice depends on the severity and symptomatology of the patient. The gold standard for the treatment of OSA is CPAP. However, compliance with a long-term treatment is limited, so we valuate alternative therapeutic methods such as Mandibular Advance Devices (DAM), and when and how to incorporate them in the treatment of this pathology.

Conclusions: The treatment of OSA requires a multidisciplinary approach, in addition to educational programs, to increase the success in the management of these patients. The studies showed a significant improvement in the quality of life of the patients who have complied with the prescribed therapeutic indications.

\section{- Oral Presentation 41}

TITLE:- Management of patients in treatment with new oral anticoagulants (NOACs).

\author{
AUTHORS: Caño Gómez N, Salido Iniesta N, Leco \\ Berrocal I, Martínez Rodríguez N, Gutierrez Sán- \\ chez L, Barona Dorado C. \\ Master de Cirugía Bucal e Implantología. Hospital Virgen de la \\ Paloma, Madrid.
}

Objetives: Update on the dental management of patients treated with NOACs.

Material and Methods: Review of literature in databases and sources of information Pubmed / Medline, Cochrane Database of Systematic Reviews and Ebsco with no published date limit, published in English or Spanish, in human patients who undergo oral interventions and are in treatment with new anticoagulants.

Results: After the identification and analysis of the articles following the inclusion criteria, there is not found an established protocol, so both protocols, the suspension and the continuation of the treatment, were analyzed. For minor surgery procedures the suspension of the NOAC dose is not recommended and for major surgery procedures, the suspension of the NOAC dose of the day of the intervention is recommended. With NOACs the appearance of late bleeding is slightly more common but easily controllable with local hemostasis.

Conclusions: The prescription of NOACs is increasing and therefore dentists should know about these new drugs, its mechanism of action and the protocol of suspension due to the lack of specific clinical guidelines. Further studies of oral interventions are required in patients in treatment with NOACs.

\section{- Oral Presentation 42}

TITLE:- The importance of an early diagnosis in premalignant oral lesions. Case report.

AUTHORS: Canaro Borría M., González Navarro B. Arranz Obispo C, Jané Salas E, López López J.

Master of Oral Medicine, Oral Surgery and Oral Implantology. Department of Odontostomatology, Faculty of Medicine and Health Sciences (Dentistry). University of Barcelona.

Introduction: Oral lesions constitute an hetherogeneous group of alterations. The importance of a differential diagnosis lies fundamentally on its prognostic projection, as well as different rates of benignity or malignancy, which will determine the therapeutical management.

Case report: A 76 year old female was referred to the HOUB (Oral Medicine, Oral Surgery and Implantology Master's Degree) by the Catalan Health Institut to as- 
sess a one year evolotion gingival lesion on the third quardrant.. The pacient presented with burning sensation and oral discomfort. After the exploration and given the clinic's characteristics, we prescribed an aqueous solution of triamcinolone acetonide $0,3 \%$ during 4 weeks. On the second appointment there was a discreet improvement in terms of discomfort, but the lesion persisted. We therefore carried out an incisional biopsy, which resulted in a moderate displasia.

Conclusions: Leucoplakia and eritroplakia are considered pre-cancerous lesions by the OMS. Multiple oral lesions, specially in the elderly, are capable of turning into invasive carcinomas. Dentists play a vital role in terms of prevention, and should know how to diagnose early stages, which will result in an adequate treatment, thus a better prognosis.

\section{- Oral Presentation 43}

TITLE:- Dental management of the patient under treatment with direct oral anticoagulants.

\author{
AUTHORS: Solís González S, Melero Alarcón C, \\ Montañés de la Fuente A, Ramírez Martínez-Aci- \\ tores, L, López-Pintor Muñoz RM, Hernández- \\ Vallejo Fernández G. \\ Postgraduate in Oral Medicine. Department of Stomatology III \\ (Medicine and Oal Surgery), Faculty of Dentistry. Complutense Uni- \\ versity of Madrid, Madrid, Spain.
}

Objectives: Oral anticoagulants are some of the most widely used drugs today, a fact that must be taken into account in dental practice, especially in the ancient population. The objective of the present study is to carry out a review of direct oral anticoagulants in comparison with the classics ones and to establish a guide for the management of anticoagulated patients during dental treatment.

Material and Methods: We conducted a literature search with studies published in English in the last 5 years, until January 2017. We searched PubMed-Medline and the Cochrane Library, using as keywords "novel oral anticoagulants", "direct oral anticoagulants", "dabigatran", "apixaban", "rivaroxaban", "dental treatment" and "dental management".

Results: Direct oral anticoagulants appear safe and effective. These drugs facilitate the management of elderly patients in the dental office and have fewer interactions and do not require periodic monitoring. In general, it is not necessary to suspend its administration for invasive dental procedures or to perform preoperative anticoagulant tests, although it is decided to handle each case individually.

Conclusions: Although direct oral anticoagulants have advantages over the classic ones, more studies are necessary to establish a procedure for the dental treatment of the elderly patient. Nevertheless, a series of recommendations can be established that allow its dental management, as long as each case is treated in a particular way.

\section{- Oral Presentation 44 \\ TITLE:- Oral squamous cell carcinoma (OSCC). Clinical case.}

AUTHORS: Tur Colomar L, Céspedes Sánchez JM, Arranz Obispo, Jané Salas E, López López J.

Máster en Medicina, Cirugía e Implantología Oral. Facultad de Medicina y Ciencias de la Salud, Odontología. Universidad de Barcelona.

Plasma cell gingivitis (GCP) is a rare oral condition characterized clinically by diffuse gingival inflammation, erythema and, in some cases, desquamation. The purpose of the review is to name the etiological factors of GCP, as well as the diagnostic criteria to carry out the appropriate treatment.

Material and Methods: We performed a review of the literature between 2010-2016 with the keywords Gingivitis of Plasma Cells and Gingivitis of Plasma Cells.

Results: We obtained a total of 60 articles in the first research carried out in the scientific platforms Pubmed and UIC. After read the title, 17 are selected and after the analysis of the extracts, according to inclusion and exclusion criteria, 3 are excluded. Thus, 16 articles meet the inclusion criteria to perform the systematic review. Discussion: The etiological factors of GCP are multiple, and may be of allergenic, neoplastic origin or unknown cause. Its diagnosis is the result of clinical examination and the anatomopathological result that should be positive for plasma cells. The treatment of this entity is controversial, the surgical approach seems to be more resolving, although new, less aggressive therapies should be investigated further.

Conclusions: The etiological factors that can cause multiple GCP, mostly unknown by the professional. The diagnosis of GCP with the biopsy of the affected region. Currently, there is no established protocol for GCP.

\section{- Oral Presentation 45 \\ TITLE:- Correlation between Apical Periodon- titis and Diabetes Mellitus. Review.}

AUTHORS: Pérez Losada F de L, Schemel Suárez M, Jané Salas E, Estruga Davesa A, Segura Egea JJ, López López J.

Oral Medicine Unit. Department of Odontoestomatology. Faculty of Medicine and Health Sciences, URF Dentistry, Bellvitge Campus. University of Barcelona. Department of Endodontics. Faculty of Dentistry. Sevilla University. 
Introduction: Apical Periodontitis (PA) is defined as an inflammatory disease of the periradicular tissues caused by persistent microbial infection in the root canal system of the affected tooth. It appears as a periradicular radiolucent area and is diagnosed by clinical criteria and radiological interpretation. Diabetes Mellitus (DM) is characterized by abnormalities in the metabolism of carbohydrates, lipids and proteins. In the literature there are several cross-sectional studies that analyze the relationship of these two entities.

Objectives: To review the scientific evidence relating chronic apical periodontitis and type 2 diabetes Mellitus.

Material and Methods: A literature review was performed in the MEDLINE, PubMed, Scopus and Cochrane databases with the term "Periodontitis apical" and "Diabetes Mellitus "as keywords. The inclusion criteria were articles published in English and Spanish, made in animals and in humans, published in the last 5 years. Levels of evidence and grades of recommendation were analyzed according to the guidelines of the Oxford Center for Evidence-Based Medicine (OCEBM).

Results: Only 15 articles were found that met the inclusion criteria. Obtaining seven animal studies and seven studies in humans, and a systematic review was included.

Conclusions: The scientific evidence suggests a common physio-pathological factor between apical periodontitis and diabetes mellitus. More prospective studies are needed to study the association between both diseases.

\section{- Oral Presentation 46}

TITLE:- Prevention of denture stomatitis in elderly patients.

AUTHORS: Díez Dans G, Rigesti Fertonani M, Moreno Quispe LA, Muñoz Corcuera M, García Rodríguez MD, Hernandez Vallejo G.

Potsgraduate in Oral Medicine. Faculty of Dentistry. Complutense University of Madrid.

Objective: The aim is the determination of the available strategies in order to prevent denture stomatitis in elderly patients taking into account its etiology.

Material and Methods: Articles were identified by a search of the PubMed electronic literature database of the last 10 years in English and Spanish with the terms "denture stomatitis", "epidemiology", "etiology" and "elderly".

Results: Denture stomatitis is a disorder characterized as inflammation and erythema of the oral mucosal areas covered by the denture. The etiology is multifactorial including lack of appropriate denture care and hygiene, continual wearing and trauma due to unstable dentures. This factors increase the ability of Candida albicans to colonize both the denture and oral mucosal surface. In addition, there are demographic factors such as the growing proportion of the older people in which the prevalence of denture stomatitis range from $15 \%$ to over $70 \%$. Inadequately oral hygiene of the elderly is not necessary to achieve oral health. In addition, there is evidence that the risk of denture stomatitis is reduced when dentures are stabilized by implants.

Conclusions: It is necessary the identification of the risk groups and the main risk factors in the etiology of the disorder. Clear instructions of the daily cleaning and minimize the trauma of the denture to the oral mucosa are some measures to reduce the frequency of denture stomatitis.

\section{- Oral Presentation 47}

TITLE:- Antibiotheraphy in odontogenic maxillary sinusitis.

AUTHORS: Barbi Actis J, Coello de la Cruz L, Santos Marino J, Rubio Alonso L, Buesa Bárez JM, Martínez González JM.

Master in oral surgery and implantology, Virgen de la Paloma Hospital, Madrid.

\section{Objectives:}

- To study the microbal flora involved in odontogenic maxillary sinusitis.

- To determine wich antibiotics are the first choice in odontogenic sinusitis.

Material and Methods: A bibliographic review of the last 10 years was carried out in the main databases, MEDLINE, Cochrane. After a screening by language (english, portuguese and spanish), tittle, content summary and number of citations there were selected 24 articles.

Results: Maxillary sinusitis secondary to an odontogenic infection (SMO) classically accounts for $10 \%$ of all maxillary sinusitis, although more recent studies revealed? true incidence of up to $40 \%$.

The bacterial flora characteristic of an SMO, both acute and chronic, has its own characteristics. It is generally polymicrobial with predominance of anaerobes, being common among these the presence of Peptostreptococcus and Prevotella ssp, and Staphylococcus Aureus and Streptococcus Pneumoniae, among the aerobes.

There is currently no "gold standard" in the antibiotic treatment of SMO, neither consensus on the real benefits of its use. Treatment should be performed after cultureand antibiogram.

Conclusions: Currently the existing studies on maxillary sinusitis of odontogenic origin do not provide evi- 
dence of a greater efficacy of treatment of one antimicrobial against another. In geriatric patients, such choice will be determined by factors such as: suceptibility of microbiota present, pharmacological interactions, adverse effects or risk of promoting bacterial resistance.

\section{- Oral Presentation 48}

TITLE:- Neurosensory complications in basic implantology. A case report in a geriatric patient.

AUTHORS: Salido Iniesta N, Marro Amador P, Leco Berrocal I, Martínez Rodríguez N, Gutiérrez Sánchez L, Barona Dorado C.

Máster de Cirugía Bucal e Implantología, Hospital Virgen de la Paloma de Madrid.

Introduction: Implant placement and its posterior rehabilitation can lead to complications that could be avoided by making a proper planification and diagnosis of the case.

The aim of the present communication is the exposition of a case report where after mandibular implant placement the patient reported a right inferior alveolar nerve paresthesia in the immediate postoperative.

Case Report: A 66 year old male patient attended to Hospital Virgen de la Paloma Oral Surgery Service willing to have implant treatment at both mandible posterior sectors.

Surgical treatment for three mandible implants was performed in the lower right quadrant and nine months after, three more implants were placed at the lower left quadrant. Right after the first procedure, the patient showed immediate paresthesia of the lower left inferior alveolar nerve, confirming the presence of the end of the implant right at the roof of the alveolar inferior nerve canal radiologically.

Therefore, the extraction of the implants was carried out and after four months, when sensitivity was recovered, new implants were placed again achieving a high rate of satisfaction of the patient.

Conclusions: A correct preoperative planification is the key to avoid neurosensorial pathology after dental implant placement.

\section{- Oral Presentation 49}

TITLE: - Comparative study on the prevalence of HPV infection in patients younger and older than 65 years.

AUTHORS: Banga González C, Gándara Vila P, Suárez Peñaranda JM, Otero Rey E, García García A, Blanco Carrión A.
Oral Medicine, Oral Surgery and Implantology Master, Santiago de Compostela University.

Summary Objectives: To study the presence of HPV in a group of healthy patients and to determine the genotypes present, as well as to analyze the influence of age and gender on infection.

Material and Methods: We carried out a descriptive study with data obtained from cytologies corresponding to 34 healthy patients in the unit of Oral Medicine, Oral Surgery and Implantology of the University of Santiago de Compostela. Samples of healthy oral mucosa were obtained by cytology on a liquid basis with cytobrush and using ThinPrep PreservCyt ${ }^{\circledR}$ Solution as a transport medium. The presence of viral DNA was carried out using the CLART®HPV 2 system (Genomica, Madrid, Spain) by polymerase chain reaction (PCR).

Results and Conclusions: Will be presented during the congress.

\section{- Oral Presentation 50}

TITLE:-Quality of life, use of dental services and accessibility of the same of the Galician population over 65 years.

AUTHORS: Durán Garnica C, Gamallo Moure A, Suárez Quintanilla J, Gándara Vila P, García García A, Blanco Carrión A.

Master's degree in Oral Medicine, Oral Surgery and Implantology at the University of Santiago de Compostela.

Goals:

- To know the use of dental services by the elderly population.

- To know the level of oral quality of life in the elderly population.

- See the influence of accessibility to health centers in relation to the oral health of the elderly.

Material and Methods: This research work has been carried out to study the oral health of the elderly population in Galicia based on the questionnaire developed and validated for the EGOHID study of European indicators.

A personal interview was conducted with 455 individuals over the age of 65 and psychologically independent. The interview consisted of 20 questions divided into 3 blocks: 6 questions of demographic data, 6 questions on the use of dental services and 8 quality of life questions.

The population was defined considering the individuals of both sexes, aged 65 or over in Galicia $(\mathrm{N}=635,879)$, the type of sampling was random stratified and a proportional affixation was made by size of habitat, age and sex.

Results and Conclusions: They will be presented at the congress. 


\section{- Oral Presentation 51}

TITLE:- Study of the pathology of TMJ and lesions of the oral mucosa in the Galician population over 65 years.

AUTHORS: Vázquez Casal A, Gamallo Moure A, Suárez Quintanilla J, Gándara Vila P, García García A, Blanco Carrión A.

Máter de Medicina Oral, Cirugía Oral e implantología. Universidad de Santiago de Compostela.

Objectives: To know the prevalence of TMJ pathology and oral lesions in the galician elderly population.

1.1 Depending on sex.

1.2 Depending on the age.

1.3 Depending on the habitat.

Material and Methods: A personal interview was conducted with 455 individuals over the age of 65 and psychologically independent. The interview consisted of 1 question about TMJ symptoms and sings. Another question is that if you have had lesions on the oral mucosa and if you have had biopsy and treatment. We used 3 variables: variable, rural-semi-urban and semiurban-urban; age, from 65 to 74 years and greater than 75 years; sex, man and woman.

The population was defined considering all people of both sexes, aged 65 or over in Galicia $(\mathrm{N}=635,879)$. The type of sampling was random stratified and a proportional affixation was made by size of habitat, age and sex. The sample error: with the confidence level of $95.5 \%$ ( 2 sigma) and $\mathrm{P}=\mathrm{Q}$ as the unfavorable case, the maximum error allowed for the sample size is $+-4.6 \%$. Results and Conclusions: They will be presented at the conference.

\section{- Oral Presentation 52}

TITLE:- Retrospective study of the clinicalpathological characteristics of oral leukoplakia in a group of patients older than 65 years old.

AUTHORS: Pena Cristóbal M, Gándara Vila P, Suárez Peñaranda JM, Otero Rey E, García García A, Blanco Carrión A.

Postgradaute Degree in Oral Medicine, Oral Surgery and Implantology. University of Santiago de Compostela.

Objective: To analyze the clinical-pathological characteristics of oral leukoplakia in a group of patients older than 65 years diagnosed clinically and histologically of this pathology.

Material and Methods: A search of clinical records was carried out with the aim of identifying patients over 65 years of age diagnosed clinically and histologically with oral leukoplakia at the Postgraduate Degree in Oral Medicine, Oral Surgery and Implantology at the University of Santiago de Compostela from January 2006 to February 2017. The following data were collected: age, sex, systemic diseases, medication, allergies, smoking and alcohol, clinical type of leukoplakia, location, size and number of lesions, treatment, follow-up time, evolution, histological characteristics and degree of dysplasia.

Results: The results will be presented at the congress. Conclusions: The conclusions will be presented at the congress.

\section{- Oral Presentation 53}

TITLE:- Study of oral health and oral hygiene in the Galician population over 65 years.

AUTHORS: Prado Pena I, Gamallo Moure A, Suárez Quintanilla J, Gándara Vila $P$, García García $A$, Blanco Carrión A.

Máster Propio en Medicina Oral, Cirugía Oral e Implantología. Universidad de Santiago de Compostela.

Objectives: To know the level of oral health and habits of oral hygiene in the Galician population over 65 years old: based on sex, age and habitat.

Material and Methods: This research work has been carried out to study the oral health of the elderly population in Galicia. We have based ourselves on the questionnaire elaborated and validated for the EGOHID study of European indicators. A personal interview was conducted with 455 individuals over 65 years. The interview consisted of 19 questions divided into 2 blocks: 6 demographic questions and 14 oral health questions and habits. Before the interview, each person signed informed consent. We used 3 variables; habitat variable, rural-semirural and semi-urban-urban; variable age, 65 to 74 years and over 75 years; variable sex, men and women. The population was defined considering all people of both sexes, aged 65 or over in Galicia $(\mathrm{N}=635,879)$. The type of sampling was random and stratified, a proportional affixation was made by size of habitat, age and sex.

Results and Conclusions: The results and conclusions will be presented at the congress.

\section{- Oral Presentation 54}

TITLE:- Psychological disturbances and perception of quality of life in patients with oral lichen planus.

AUTHORS: Vilar Villanueva M, Blanco Aguilera E, Gándara Vila P, Otero Rey E, García García A, Blanco Carrión A. 
Máster de Medicina oral, Cirugía oral e Implantología. Facultad de Odontología, Universidad de Santiago de Compostela.

\section{Objectives:}

1. To determine the influence of psychological alterations, anxiety and depression, in the diagnosis of oral lichen planus (OLP).

2. To define the quality of life in OLP patients.

Material and Methods: Fifty patients over the age of 65, diagnosed clinically and histologically of OLP were selected. Anamnesis was performed and a blood test was requested. Then, the HADS and OHIP-14 tests were completed to assess anxiety, depression and the perception of quality of life.

Results: $68.38 \%$ of the patients with OLP present anxiety and, $94.7 \%$, depression. White OLP patients obtained higher scores of anxiety and depression than patients with the red type, although no statistically significant differences were found. In addition, there is a trend towards a worse quality of life perception in patients with red OLP than in those with white OLP.

\section{Conclusions:}

1. Psychological disturbances are frequently related to OLP. There is no influence on the clinical type of oral presentation.

2. There is a slight alteration in the perception of quality of life in our OLP patients. Although this alteration is greater in those with red lesions, there is no statistical significance.

\section{- Oral Presentation 55}

\section{TITLE:- Lichen planus as a predictive factor of} cardiovascular disease risk.

\author{
AUTHORS: Yáñez Busto A, Baña Souto S, Otero \\ Rey E, Gándara Vila P, García García A, Blanco \\ Carrión A. \\ Master of Oral Medicine, Oral Surgery and Implantology of the Uni- \\ versity of Santiago de Compostela.
}

Objectives: To study the possible relationship between the inflammation produced by the immune reaction that occurs in the OLP and the inflammatory response caused in cardiovascular diseases.

Material and Methods: We studied a group of 58 patients clinically and histologically diagnosed of OLP. All of them are asked to perform a complete blood analysis. The results are compared with respect to a control population and the clinical type of OLP. A bibliographic search was performed at Pubmed / Medline database, which was limited to the serum concentration of cardiovascular risk biomarkers in OLP patients.

Results: The results show elevated serum lipid concentrations in OLP patients compared to the control group.
The reviewed literature shows that the serum concentration of cardiovascular risk biomarkers is higher in OLP patients.

Conclusions:

1. We observed that the only biochemical alteration significantly associated with OLP was the presence of hypercholesterolemia.

2. The inflammatory process that occurs in LPO could explain its relationship with dyslipidemia and elevated serum concentration of cardiovascular risk biomarkers. 3. Although more studies are needed, our results suggest that we could use lipid profile analysis as a predictor of cardiovascular risk in OLP patients.

\section{- Oral Presentation 56}

TITLE:-Quality of life in geriatric patients with burning mouth syndrome.

AUTHORS: Rodríguez Vázquez P, Suárez Quintanilla JA, Pérez López D, Suárez Alén F, Peñamaría Mallón M, Blanco Carrión A.

Máster en Patología Médico Quirúrgica y Odontología integral. Facultad de Odontología, Universidad de Santiago de Compostela.

Objective: The objective of present study was asses the perception of life in pacients diagnosed of burning mouth syndrome.

Material and Methods: 25 patients over 65 years of age diagnosed with Burning Mouth Syndrome in the Oral Medicine, Surgery and Implantology Master's (Faculty of Dentistry, University of Santiago de Compostela) were compared with a control group of 25 health patients with the same distribution in age and sex. The validated test OHIP-14 (The Oral Health Impact Profile 14)was used to evaluate general quality of life, .

Results and Conclusions: Will be presented during the exhibition of the oral communication

\section{- Oral Presentation 57}

TITLE:- Impact of Oral Medicine on knowledge about oral cancer in Dentistry students.

AUTHORS: Barba Montero C, Suárez Quintanilla JA, Pérez López D, Suárez Alén F, Peñamaría Mallón M, Blanco Carrión A.

Máster Patología Médico-Quirúrgica Oral y Odontología Integral. Facultad de Medicina y Odontología. Universidad de Santiago de Compostela.

Objectives: To assess the impact of oral cancer knowledge on the professional attitude of dental students.

Material and Methods: A descriptive study was car- 
ried out in the students of the Degree of Dentistry of the University of Santiago de Compostela, which were divided in two groups, one of students of 1st and 2nd year who had not attended any clinical course and another group. That yes he had taken the subject of Oral Medicine. All of them were given a questionnaire in which the following parameters were collected: course, sex, age and 8 items on knowledge of oral cancer. All data were entered into a database and analyzed using SPSS 2.0 software for Windows (SPSS, CHICAGO, IL, USA).

Results and Conclusions: will be exposed during the Congress.

\section{- Oral Presentation 58}

TITLE:- Study of the prevalence of xerostomia in geriatric patients.

AUTHORS: Rodríguez Díaz C, Suárez Quintanilla JA, Pérez López D, Suárez Alén F, Peñamaría Mallón M, Blanco Carrión A.

Master's Degree in Medical-Surgical Pathology and Integral Dentistry. Faculty of Medicine and Dentistry, University of Santiago de Compostela.

Objectives: To know the presence of xerostomia symptoms in an elderly Galician population and asses the influence of age, sex, habitat and clinical data.

Material and Methods: To make this study we have based ourselves on the questionnaire elaborated and validated for the EGOHID study of European indicators.

A personal interview was conducted with 455 patients over the age of 65 and psychologically independent. The interview consisted of 11 questions related to the perception of xerostomia in different habits of daily living. Prior to the interview, each person signed informed consent. It was considered 3 variables: variable habitat, variable age and variable sex.

The population was defined considering all people of both sexes, aged 65 or over in Galicia $(\mathrm{N}=635,879)$. The type of sampling was random stratified and a proportional affixation was made by size of habitat, age and sex. The sample error, with a confidence level of $95.5 \%$ (2 sigma) and $\mathrm{P}=\mathrm{Q}$ as the worst case. The maximum error allowed for the sample size is $\pm 4.6 \%$.

Results and Conclusions: They will be exhibited during the congress

\section{- Oral Presentation 59}

TITLE:- Attitudes of dental students in Galicia toward tobacco-use cessation counseling.
AUTHORS: Lorenzo Pouso AI, Suárez Quintanilla JA, Pérez López D, Suárez Alén F, Peñamaría Mallón M, Blanco Carrión A.

Master en patología médico-quirúrgica oral y odontología integral, Facultad de Medicina y Odontología, Santiago de Compostela.

Objectives: The aim of this study was to describe the habits of tobacco use, dependence and motivation to stop smoking in dentistry students. The aim was also to analyze their knowledge about the consequences of smoking in their patients and their attitudes toward tobacco-use cessation in dental practice.

Material and Methods: A questionnaire was distributed to the students of all the courses of the faculty. This questionnaire had three parts. The first part was about students' knowledge of the impact of tobacco on health and the opinions of students in providing help to their patients to quit smoking. The following parts were designed to know the consumption of tobacco among students as well as their dependence on nicotine and their motivation to give up the habit.

Results and Conclusions: The results are for the moment in statistical analysis. They will be presented during the congress.

\section{- Oral Presentation 60 TITLE:- Study of gustatory preference in geria- tric patients.}

AUTHORS: Piñeiro Donís S, Suárez Quintanilla JA, Pérez López D, Suárez Alén F, Peñamaría Mallón M, Blanco Carrión A.

Master's Degree in Medical-Surgical Pathology and Integral Dentistry. Faculty of Medicine and Dentistry, University of Santiago de Compostela.

Objectives: To know the gustatory preference in the elderly Galician population in relation to its age, sex and habitat.

Material and Methods: This research work has been carried out to study the oral health of the elderly population in Galicia. We have based ourselves on the questionnaire elaborated and validated for the EGOHID study of European indicators. A personal interview was conducted with 455 patients over the age of 65 and psychologically independent. The interview consisted of 4 questions related to gustatory preference over sweet, acidic, bitter and salty foods. Before the interview, each person signed informed consent. Three variables were considered: habitat variable, age variable and sex variable. The population was defined considering all people of both sexes, aged 65 or over in Galicia $(\mathrm{N}=635,879)$. The type of sampling was random stratified and a proportional affixation was made by size of habitat, age and 
sex. The sample error, with a confidence level of $95.5 \%$ (2 sigma) and $\mathrm{P}=\mathrm{Q}$ as the worst case. The maximum error allowed for the sample size is $\pm 4.6 \%$.

Results and Conclusions: They will be exhibited during the congress.

\section{- Oral Presentation 61}

TITLE:- Reasons for Consultation in Primary Care in Dentistry.

AUTHORS: Ponte Calvo F, Suárez Quintanilla JA, Pérez López D, Suárez Alén F, Peñamaría Mallón M, Blanco Carrión A.

Máster Patología Médico-Quirúrgica Oral y Odontología Integral. Facultad de Odontología. Universidad de Santiago de Compostela.

Objectives: Evaluate and compare the visit patterns of the first dentistry visits in Primary Care, analyzing the reason for the visit and comparing it with the definitive diagnosis of the patient.

Material and Methods: We performed a study to analyze the frequency of reasons for consultations and the diagnosis of Dentistry in Primary Care. We have developed a list of possible reasons for consultation in all patients seen during a month, establishing them on a scale of 7 items. Similarly, a list of possible diagnoses has been designed in these same patients, elaborating a scale of 8 items. Subsequently, a total of 562 patients were treated and the reasons for consultations and diagnoses were analyzed only to those who came for the first time.

The validity of the list of reasons for consultation and diagnosis in Primary Care Dentistry has been verified. We have analyzed the number of first consultations, frequency of reasons for consultation, and frequency of diagnoses in Primary Care Dentistry in relation to age and sex.

Results and Conclusions: Will be exposed during the development of the congress.

\section{- Oral Presentation 62}

TITLE:- Oral lichen planus and dental implants. Case report.

\author{
AUTHORS: Mateo-Sidrón Antón, M.C., López Sán- \\ chez A.F. \\ Facultad CC. de la Salud. U. Rey Juan Carlos. Madrid.
}

Introduction: Oral Lichen Planus is an inmunologic and cronich inflammatory disease which affects skin and mucus membranes, specially the oral mucosa $(80 \%)$. It develops with periods of exacerbation and remission.
It's etiology is unknown and affects $1-2 \%$ of the population. The indication of placing dental implants in patients with OLP has been studied, causing controversy as some authors believe its contraindicated, because it can also boost the appearance of oral carcinoma, while other authors think otherwise.

Clinical Case: A 77 year old woman, diagnosed of OLP in 2011. In September of 2016 she comes to a checkup presenting pain and burning-like symptoms in the gingiva mucosa in IOI 35-36 which are on a reticular lesion of OLP. The patient did not have clinical nor radiographic findings, that's why it was prescribed topical corticosteroid mixed with antifungical therapy in orabase, for three weeks. Although the patient felt some improvement, she still felt pain when under stress and felt leftover food under the implant supported crowns. After changing the crowns and upgrading the oral higiene techniques, the patient does not present any symptoms.

Conclusions: Although OLP does not seem to be a contraindication for placing implants, is really important to monitorize each patient in a periodontal aspect, treat OLP in a farmacologic way when it is indicated and maintain an excelent plaque control so that we can avoid the irritation factors that act upon the healthy mucosa, specially in patients who are diagnosed of OLP which have a primary alteration.

\section{- Oral Presentation 63}

TITLE:- Neuropathic oral-facial pain. Case study.

\author{
AUTHORS: Martínez Puga M, Arnedo Martínez \\ AM, Santos Marino J, Rubio Alonso R, Barona Do- \\ rado C, Martínez González JM. \\ Hospital Virgen de la Paloma Oral surgery and dental implantho- \\ $\log y$ M.A.
}

Introduction: Neuropathic pain is defined as pain unrelated to the activation of pain reception cells in any place of the living organism. It is caused by an injury or disease of the somatosensory system.

It is a neuropathy with pain located at the oral-facial area.

Clinical Case: Female patient, 65 years old with pain on the third quadrant attributed to the necessity of root canal therapy and dental extraction. After performing of both no improvement was observed in the patient's symptomatology with appearance of oral bitterness and metallic taste plus itch and pain on the left side of the tongue.

After a diagnosis of a possible neuralgia on the median nerve based on a electromyography performed by a doctor, the patiente seeks for several different medical 
opinions. All the other doctors agree on the diagnosis of an oral dysaesthesia on the lingual nerve and left inferior alveolar nerve.

The treatment prescribed was based on the combination of several different drugs:

Neurotin 300mg 1 every $24 \mathrm{~h}$

Arcoxia $90 \mathrm{mg} 1$ every $24 \mathrm{~h}$

Espidifen $600 \mathrm{mg} 1$ every $8 \mathrm{~h}$

Afterwards she was treated via pulsed radiofrequency keeping also the pharmacological treatment with no improvement observed.

Conclusions: As observed in this case, neuropathies are hard to diagnose, and thus require complex treatments. 


\section{PÓSTERS}




\section{- Poster 1}

TITLE:- Prophylaxis of infective endocarditis. Systematic review.

AUTHORS: López Fernández M, Márquez Sánchez S, Benarroch Cohen S, Olivan Molina S, Fernández Domínguez M.

Máster Universitario en Cirugía Bucal e Implantología, Universidad San Pablo CEU/Master's Degree in Oral Surgery and Implantology, CEU San Pablo University.

\section{Objectives:}

1. Define whom patients is indicated prophylaxis of infective endocarditis.

2. Delimit in which treatments is used this prophylaxis. 3. Specify how to do it.

Material and Methods: Bibliographic searched in Pubmed, Ebsco and Cochrane.

Search criteria: year of publication (2006-2015) and keywords: infective endocarditis, oral surgery and endocarditis prophylaxis.

Twelve articles were selected, eleven were systematic reviews and one, a clinical study.

Two articles were discarded because the information contained in them wasn't relevant.

Results: Different protocols indicate that prophylaxis for infective endocarditis is necessary in certain dental treatments and will carried out with amoxicillin as the first choice.

However, there are two protocols in which we find some arguments:

- The British Society for Antimicrobial Chemotherapy (BSAC) indicated that $3 \mathrm{~g}$ of amoxicillin were required for prophylaxis, instead of $2 \mathrm{~g}$ as is indicated for other organisms.

- The National Institute for Health and Care Excellence (NICE) noted that prophylaxis was not necessary in dental treatments, however other protocols indicate that it is necessary.

\section{Conclusions:}

1. Patients to whom prophylaxis should be indicated are:

a. Previous Infective Endocarditis.

b. Valvular prosthesis.

c. Serious congenital heart disease.

d. Heart transplantation that develop some valvulopathy.

2. Prophylaxis is recommended whenever it is performed

a. Manipulation of the gingival tissue and the periapical region.

b. Perforation of the mucosa.

3. Prophylaxis will be:

a. First choice: Amoxicillin ( $2 \mathrm{~g} / \mathrm{h}$ before).

b. Allergic to beta-lactams:

i. Clindamycin ( $600 \mathrm{mg} / \mathrm{lh}$ before).

ii. Azithromycin or clarithromycin $(500 \mathrm{mg} / 1 \mathrm{~h}$ before).

\section{- Poster 2}

TITLE:- Torus mandibularis and palatinus.

AUTHORS: Benarroch Cohen S, Fernández-Nespral Loring V, Moreno Bresme A, Olivan Molina S, Fernández Domínguez $M$.

Máster en Cirugía Bucal e Implantología. Universidad San Pablo CEU. Madrid.

Introduction: Oral tori are benign, slow growing, osseous excrescencies, that usually manifest in young adults. These bone growths usually do not require treatment, but in some cases surgical removal will be necessary.

\section{Objectives:}

1. To describe oral tori and their etiology

2. To name the types of tori accordind to their location

3. To mark out the cases in which surgical intervention is necessary

Material and Methods: Bibliographic search in Ebsco y Pubmed, between the years 2013 and 2016, with key words: torus mandibulares, torus palatinos, $y$ oral tori.

Results: Oral tori, covered by a verry thin mucous tissue, usually produce ulcers in patients carying removable dentures. Pacients presenting these oral exostosis usually suffer from palatal and sublingual lesions due to constant trauma produced by pointy foods falling upon the thin layer of tissue that covers these osseous lesión. Conclusions: Tori are uni or bilateral, circunscribed, well delimited, benign osseous tumors, of multifactorial etiology (genetic and environmental). There are two types of tori according to its localization, torus mandibularis and torus palatinus. Oral tori, in most cases do not need treatment, but when they become too big, produce traumatic ulcers due to food while chewing, bother aesthetically, or they interfere with dental prostheses, these will need to be removed by means of a surgical opening and osteoctomy.

\section{- Poster 3}

TITLE:- Influence about intravenous sedation in clinic results of implants surgery.

\section{AUTHORS: Acuña Pedrosa JM, Duarte Díaz F, Ga- llego Alonso JI, Gómez Valderrama R. \\ Málaga University: Master on Oral Surgery, Implantology and Periodontics.}


Material and Methods: A monitoring was done after $24 \mathrm{~h}$, fifteen days later, and eventually monthly to every patient of both groups. Those patients that were not under monitoring were excluded. There was a valoration about post-procedure inflammation by means of descriptive scale, satisfation of the patients on a survey, intensity of pain by quantitative scale ( soft/ mild / strong pain ) and rates on unsuccessful implants.

Results: There is a greater satisfaction in proceeding with a group $\mathrm{S}\left(\mathrm{P}=0^{\prime} 03\right)$, with a lower intra oral inflammation, as well as less impact of mild/strong pain postprocedure and a rate of unsuccessful implants with no statistically significance between both groups.

Conclussions: We think that the lower inflammation and intensity of the post-procedure pain is related to the use of corticoids and analgesics (iv) as a treatment of the anesthetics doctors during the surgery, which results in an high level of satisfaction in the patient without affecting the unsuccessful implants rates.

\section{- Poster 4}

TITLE:- Bibliographic review on oral lichen planus and implants.

AUTHORS: Gómez Valderrama R, Duarte Diaz F, Acuña Pedosa JM.

Master Cirugia oral, implantes y periodoncia. Universidad de Malaga. (UMA).

Aim: The aim of this study is to evaluate what is written in the literature on oral lichen planus (OLP) and implants, and to assess the survival of implants rehabilitation supported in these patients.

Material and Methods: A Medline search was carried out using the keywords "oral lichen planus", "dental implants" and "desquamative gingivitis and implants.

Results: We found two reviews (Petruzzi $\mathrm{M}$ et al and Candel-Martí E et al) where they did not find differences in the survival rate between patients with OLP treated with implants and patients treated with implants without the disease. Lopez-Jornet et al. carried out a study in which they controlled the quality of life, which was better in patients with OLP treated with implants. We have only found one article (Hernandez $\mathrm{G}$ et al) where they relate the presence of desquamative gingivitis with an increased risk of presenting peri-implant mucosistis. Petruzzi et al suggest that the causes of implant loss in the studies were parafunctions, poor bone quality and mandibular resections due to oral squamous cell carcinoma (OSCC).

Conclusions: Patients with OLP and treated with implants have a very high rate of success. Regular control of the disease and implants is essential, as it should not be forgotten that LPO has a malignancy rate of $1 \%$.

\section{- Poster 5}

TITLE:- Baroque music as a control of anxiety in adult dental patients: Hearing sensory marketing.

AUTHORS: Gómez Font R., Povedano Jiménez MA, Gómez Sánchez M, Suárez García A, Aguado Gil JM, Muñoz Leal MJ.

Facultad Ciencias Biomédicas. UEM, Facultad Ciencias Sociales y de la Comunicación. UEM.

Objectives: To determine if baroque music is determinant in the anxiety state of the adult dental patient versus other sound stimuli (silence or general radio music) in order to be used as a tool for sensory marketing.

Material and Methods: A total of 150 patients aged from 20 to 85 were selected, then assigned different types of aural stimuli: silence, general radio music and baroque music. After 5 minutes in the waiting room, subjected to the desired stimulus, they were given a STAI questionnaire.

Results: When we applied a Wilcoxon signed-rank test, we found that there were no significant differences in the before and after DECA in the case of silent waiting. However, a level of significance of 0.05 allows us to reject the null hypothesis of equality of means in the before and after DECA for patients who listened to the radio or baroque music while waiting, obtaining a better level for baroque music and in patients older than 60 years.

Conclusions: As a result of this we can affirm that baroque music is the sound stimulus that most reduces anxiety of the three stimuli tested, generating a differentiating consumption experience making it a valid marketing tool.

\section{- Poster 6}

TITLE:- Osseointegration of oral implants in elderly patients. A literature review.

AUTHORS: Cisnal San Juan M, Fernández Domínguez M, Aguilar Martín C, Pérez Corral I, Cabezas Mojón J.

Máster en Cirugía Bucal e Implantología. Universidad San Pablo CEU. Madrid.

Purpose: The aim of this article was to review the literature of successful osseointegration of dental implants in elderly patients.

Material and Methods: A review of the literature of the last 20 years of national and international articles is carried out. The electronic bibliographic sources Medline Pub-Med and Web of Science are used, using as 
keywords "oral implantology", "osseointegration", "geriatric patient" and "geriatric dentistry".

Results: There is no evidence that aging has negative effects on the long-term results of osseointegrated oral implants. Osseointegration is not affected by the advanced age of the patients. In this sense, bone and mucosal healing after surgical insertion of implants is not a major problem among elderly patients.

Conclusion: According to this breaf bibliographic research it can be concluded that old age does not seem to represent a factor of major prognostic significance in treatment with dental implants.The integral evaluation (oral and systemic) in elderly patients is important. Overdentures with osseointegrated implants provide a high degree of satisfaction among elderly edentulous patients, since it constitutes an essential change from a situation of total edentulism to a semi-edentulous state with greater well-being, comfort, esthetics and function.

\section{- Poster 7}

TITLE:- Oral mucositis in oncology patients.

\author{
AUTHORS: Sánchez Zorrilla A, Cabezas Mojón \\ J, López Fernández M, Pérez Corral I, Fernández \\ Domínguez M. \\ Master's degree in Oral Surgery and Implantology. San Pablo CEU \\ University. Madrid.
}

Indroduction: Mucositis appears as a side effect of radiotherapy and / or chemotherapy treatments. It's an inflammatory reaction that affects the oropharyngeal mucosa. Its prevalence is $30-85 \%$. It can cause ulcers that produce intense pain and difficulties to eat and drink, which may need the administration of analgesic opiates, hospitalization and nasogastric/intravenous nutrition. These complications can interrupt or alter the treatment of cancer.

Objective: Reviewing the literature to find out about oral mucositis and its management.

Material and Methods: Bibliographical review made using the search engines Pubmed and Cochrane. 13 articles were selected. Keywords used were: "oral mucositis", "radiotherapy", "chemotherapy", "management" and "treatment".

Results: The oral mucosa presents high levels of mitotic activity and cell turnover, which makes it more sensitive to the action of radiotherapy and chemotherapy. It appears mostly on the lips, floor of the mouth, soft palate and lateral aspect of the tongue. There seems to existsome risk factors associated with its appearance, related mainly to the type of oncological treatment and with some individual factors of the patient.
Conclusions: An odontology control must be realized before, during and after the beginning of the oncological treatment.

The treatment is mainly palliative, easing the pain of the patients with analgesic and local anesthesia.

They must support to keep the mouth wet and cleanin order to prevent any infection or periodontal complication.

It's recommended a soft diet, low in carbohydrates and low intake of acid, spicy or corse food.

Mouthwash containing alcohol must be avoid.

\section{- Poster 8}

\section{TITLE:- Bibliographical review on the use of acupuncture in oral surgery.}

\section{AUTHORS: Cordero Ayala A, Gómez Valderrama R. \\ Master of oral surgery, implants and periodontics. University of Malaga (U.M.A).}

Aim: Acupuncture is part of traditional Chinese medicine, used to treat systemic or local pathologies by inserting needles at certain anatomical points; the acupuncture's effectiveness is continuously in question, so many authors have carried out studies to verify its validity. Our objective with this literature review, is going to be if the different studies show that acupuncture is really useful to use in oral surgery.

Material and Methods:We searched pubmed, researchgate, google academic, with keywords: "acupuncture and dentistry", "acupuncture and oral surgery", "acupuncture and analgesia", "acupuncture and pain control", "Acupuncture and anesthesia", "acupuncture and implantology", "acupuncture and paresthesia"

Results: We analyzed 21 articles: 3 about preoperative and intraoperative anxiety control (e.g., Rosted, Palle inferred that $60 \%$ of treated patients decreased anxiety), 3 postoperative analgesia, 3 studied intraoperative anesthesia, 2 talked about improving Saliva flow, 3 on TMJ damage, 2 on nausea control, 2 articles on smoking cessation, and 3 on paresthesia. High percentages of success were found in the control of nausea, anxiety, TMJ treatment and xerostomia, in relation to conventional treatments; In terms of analgesia and anesthesia, are more difficult to assess because of the subjectivity to which they are exposed. Also to highlight the few adverse effects, appearing mainly derived from malpractice.

Conclusions: It would be convenient to incorporate acupuncture as an adjunct in traditional techniques; Interesting in the treatment of medically compromised patients, for their safety. 


\section{- Poster 9}

TITLE:- Eosinophilic ulcer. Case report and review of the literature.

AUTHORS: González Moreno E, Gallardo Castillo I, Caballero Aguilar J, Vigo Martínez M, MartínezSahuquillo Márquez A.

Facultad de Odontología de la Universidad de Sevilla.

Introduction: Eosinophilic ulcer is a rare variety within chronic ulcers of the oral mucosa. It is a benign lesion with unknown etiopathogenesis. Clinically, this lesion manifests as an isolated ulcer, with raised and indurated borders, with a white or yellowish background, which may resemble a malignant lesion or an oral manifestation of some infections. Microscopically it is characterized by a diffuse inflammatory infiltrate of polymorphonuclear cells rich in eosinophils, enveloping the more superficial mucosa and the deeper muscular layer. This paper presents a clinical case and a review of the scientific literature on the most important aspects regarding the diagnosis and treatment of this pathology.

Case Report: A 68-year-old patient, who reported an ulcer lasting more than one month. The diagnosis of suspicion is of traumatic ulcer and the differential diagnosis is against oral squamous cell carcinoma. After an incisional biopsy, the lesion was resolved.

Conclusions: Eosinophilic ulcer is a rare benign chronic lesion, with lingual localization preferentially. The clinical history and the anatomopathological study will give the definitive diagnosis. In practice, biopsy surgical intervention induces the lesion to heal, although other authors suggest an expectant attitude.

\section{- Poster 10}

TITLE:- Ackerman's Verrucous Carcinoma: A case report.

\author{
AUTHORS: Bornachea Urbina M, De la Sen Cor- \\ cuera O, Cisnal San Juan M, Cabezas Mojon J, \\ Domínguez Fernández $M$. \\ Master in oral surgery and implantology. San Pablo CEU Univer- \\ sity. Madrid.
}

Introduction: Ackerman's Verrucous Carcinoma is a lower incidence variant of the well differentiated squamous cell carcinoma. Clinically the lesión appears as a thick, diffuse plate, well delimited and painless. Within the oral cavity, it is usually located in the mucosa, gingiva, palate and floor of the mouth. They are observed predominantly in elder men and are associated with the consumption of tobacco, poor oral hygiene, unadapted prosthesis and Human Papilloma Virus. It is a slow growth tumor and metastases are rare. The most appropriate management is early diagnosis and surgical removal of the lesion.

Case Clinical: We present the case of a patient, 97 years old, who attends inquiry by an ulcerated lesion in the tongue of 20 days of evolution. After performing a biopsy, Ackerman Verrucous Carcinoma is diagnosed. It covers the floor of the mouth, right side edge of the tongue and right buccal mucosa.Because the age of the patient and extension of the lesión, surgery and radiotherapy are dismissed, and treatment is managed with Methotrexate.

Conclusions: According to Yoshimura et al. (2001) surgery is the most reliable method of treatment for the Verrucous Carcinoma. It uses to be treated by surgery or radiotherapy. In this case, that option would affect the patient's quality of life by the mutilation that surgical treatment would entail.In the last years, other pharmacological alternatives have been proposed, such as Methotrexate. This is an antineoplastic and immunosuppressive drug that interferes with cell processes of replication and reparation.

\section{- Poster 11}

TITLE:- Mucograft as an alternative to grafts in prosthetic rehabilitation or mucosal defects.

AUTHORS: Moreno Bresme A, De La Sen Corcuera O, Bornachea Urbina M, Cabezas Mojón J, Fernández Domínguez M.

Máster en cirugía oral e implantología. Universidad CEU San Pablo.

Objectives: To describe the histological and clinical properties of Mucograft (MC) when used as a substitute for the Connective Tissue Graft (GTC) in the correction of defects of the alveolar mucosa.

Material and Methods: PubMed search using the keywords: "Collegen Matrix" "Connective Tissue", "Recession" and "Healing".

Results: Biomaterial that integrates perfectly in the surrounding tissues. It consists of two surfaces that cause different reactions: a porous layer that allows an abundant cellular infiltration, and another compact layer that does to a lesser degree. There is no evidence of adverse tissue reaction since $90 \%$ is collagen that fails to activate the host immune response due to the structural similarity of the collagen between species. It results in the formation of scarce granulation tissue that is responsible for revascularization.

Conclusions: The use of Mucograft reduces morbidity in the geriatric patient by avoiding a second surgical area which reduces the time of the procedure obtaining similar results to those obtained with the GTC. 


\section{- Poster 12}

TITLE:- Root caries prevention protocol whit hight concentration of fluor varnish: Reality or utopia?

\author{
AUTHORS: Ortolá Siscar JC, Montiel Company \\ JM, Almerich Silla JM. \\ Departamento de Estomatología. Universitat de Valencia.
}

Introduction: The efficacy of topical fluorides in reducing caries risk as well as stopping incipient lesions has been amply demonstrated in available scientific evidence.

Older patients keep more and more natural teeth in the mouth and our mission as professionals is to help them keep them as long as possible with acceptable functionality. With age, the patient's own plaque control and oral hygiene measures have been shown to deteriorate, and professionals must establish the appropriate guidelines to reduce the risk of root caries, despite the fact that the control of plaque is poor. For this, it is essential to include these patients in prevention programs with specific protocols based on their individual risk of caries (high, moderate or low).

Clinical Cases: Since years we have been applying customized protocols of application of fluoride varnish of high concentration in elderly patients considered to be at high risk for caries and with incipient lesions of root caries. We demonstrated by the photographic follow-up of root caries lesions that they have paralyzed their evolution and that the appearance of new lesions has been practically insignificant in a 20 -year period for the longest-lived cases.

Conclusions: The prevention and stopping of non cavitated root caries is possible thanks to the application of highly concentrated fluoride varnish (Duraphat R).

\section{- Poster 13}

TITLE:- Alveolar preservation through a facial shiedl tecnique: A case report.

AUTHORS: Domínguez Moreno FJ, Robles Cantero D. Universidad Rey Juan Carlos I.

Introduction: The extraction of single as well as multiple teeth induces a series of adaptative changes that affect all of periodontal tissues. Prevention of alveolar ridge deformities aim to preserve the resorption of hard and soft tissues in the edentulous ridge in order to provide better conditions on the site for the future implant installation. Different surgical techniques have been proposed to prevent and minimize ridge collapse after tooth extraction that could obtain higher or fewer success.
Case Report: In this paper we present an alveolar ridge preservation case, through modified socket shield technique. While leaving the facial shield for a 3 month delayed implant placement, we managed to evaluate the osseous regeneration beetwen palatal cortical and facial shield as well as dimensional changes in vestibular wall using CBCT.

Conclusions: We concluded that this technique could be an easy, cheap and predictable surgical technique, in which dimensional changes in the vestibular wall were not observed getting full osseus regeneration beetwen palatal cortical and facial shield.

\section{- Poster 14}

TITLE:- Review literature on the uses of them cells mother in the field oral.

\section{AUTHORS: Mora Castilla, D.}

Máster de Cirugía Oral, Implantes y Periodoncia. Universidad de Málaga (U.M.A.).

Objectives: Stem cells have a potential for unlimited multidifferentiation, thus being an innate source for regenerative medicine, since cell renewal mechanisms can be used to repair damaged tissues or even to generate new ones. In this literature review we intend to conclude whether the different studies have indeed proved useful in this field.

Material and Methods: We searched Pubmed, Google academic, with keywords: "Stem Cells", "Regenerative Medicine", "Oral Medicine".

Results: We analyzed 20 articles: 4 on the different sources of stem cell extraction; both embryonic and adult, 5 of them on the applications in the improvement of the periodontal treatment and 11 of them on the multipotentiality and pluripotentiality of the stem cells and their dental use. Finding high percentages of success as far as regeneration is concerned.

Conclusions: Although there is still a long way to go, current studies give us a favorable prognosis regarding the use of stem cells as a precursor of bone regeneration and, consequently, its application to oral surgery.

\section{- Poster 15}

TITLE:- Early loading with mandibular overdenture by implants with acid-etched surface. Longterm-results.

AUTHORS: Jimenez Martin I, Escuin Jiménez P, España Guerrero E, Matos Garrido N, Ortiz García I, Velasco Ortega E.

Master of Implant Dentistry, Faculty of Dentistry, University of Seville. 
Introduction: Early loading protocols have improved the quality of life of implant patient. Overdentures have represented a very favorable oral restorative option in edentulous patients. The main goal of this work is to present a clinical case report where we integrate different implant techniques that form early loading of dental implants with a mandibular overdenture

Case Report: A 64-year-old woman, without relevant medical background. To the clinical exploration, it observe that is an edentulous patient with complete denture prosthesis. An orthopantomography is performed and we consider making a mandibular overdenture by implants. We insert four $4 \times 12 \mathrm{~mm}$ TSA Defcon implants with internal connection and acid-etched surface. Surgical insertion was realized using conventional surgical technique with supracrestal flap, and nonsubmerged technique. 6 weeks later, we do the early loading by the placement of a bar attachment with an overdenture. The follow-up period was 15 years after loading without surgical or prosthodontic complications. We have been replaced the plastic retainers for holding the prosthesis to the bar attachment 3 times.

Conclusions: Early loading of dental implants with overdenture supported by a bar attachment can be an alternative of implant treatment that improves functional and aesthetic expectations of edentulous patient with a high success in the long-term.

\section{- Poster 16}

\section{TITLE:- Maxillary fixed supported-implant re- habilitation in geriatric patient.}

\author{
AUTHORS: Ruiz Garrido R, Fernandez Garcia N, \\ Garcia Navarro JR, Nuñez Marquez E, Moreno Mu- \\ ñoz J, Velasco Ortega E. \\ Master of Implant Dentistry, Faculty of Dentistry, University of \\ Seville.
}

Introduction: Fixed oral rehabilitation represents a modality of treatment with implants in those patients with a long-term total edentulism. The objective of the present clinical case was to show the evaluation of the fixed rehabilitation on implants in a edentulous maxillary geriatric patient.

Clinical Case: Male of 70 years, with no medical history of interest. The clinical examination shows that the patient is a total edentulous patient with a complete prosthesis. A conical beam computerized tomography is performed and the possibility of performing a fixed oral rehabilitation on implants is considered. Eight Ga-

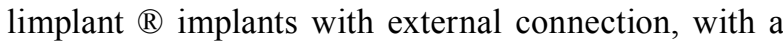
sand-blasted and acid-etched surface, are inserted. The insertion was performed by conventional surgical technique with supracrestal flap, and submerged technique.
At 3 months, the functional loading was performed by placing a cemented oral rehabilitation. The follow-up period was 5 years after loading without surgical or prosthodontic complications.

Conclusions: Fixed oral rehabilitation on implants can be an alternative implant treatment that improves the functional and aesthetic expectations of the total edentulous patient with a high success in the long term.

\section{- Poster 17}

TITLE:- Mandibular overdenture retained with locator by two implants in geriatric edentulous patient.

AUTHORS: Martí Villaescusa A, Garcia Sánchez A, Garcia Tirado A, Matos Garrido N, Ortiz García I, Velasco Ortega E.

Master of Implant Dentistry, Faculty of Dentistry, University of Seville.

Introduction: Mandibular overdentures have proved a very favourable option for oral rehabilitation of totally edentulous geriatric patients. The aim of this document is to present a clinical case involving implant techniques with early loading of dental implants and mandibular overdenture.

Clinical Case: Female 78 years, with no relevant clinical history. Under clinical observation it was observed that the patient is totally edentulous and wears a complete denture. An orthopantomography was carried out and the possibility of implementing mandibular overdenture on supporting implants was considered. It inserted two Galimplant ${ }^{\circledR} 4 \times 12 \mathrm{~mm}$, external connection implants, with sanded and acid-etched surface. The surgical insertion was performed following conventional surgical techniques with flap, and submerged technique. After 6 weeks, early loading was practiced, with a mandibular overdenture by Locator type anchors. There was a 5 year follow-up period since the loading was applied, during which no surgical or prosthetic complications were observed.

Conclusions: Early loading of dental implants with overdenture supported on frictional attachments may represent an alternative to implant treatment improving functional and aesthetic expectations of totally edentulous patients with high long-term success rates.

\section{- Poster 18}

TITLE:- Immediate loading of postextraction implants in the anterior mandibular area of a geriatric patient. 
AUTHORS: Romero Reyes J, Lago Pacheco E, Mesa Sanchez C, Matos Garrido N, Ortiz Garcia I, Velasco Ortega E.

Master of Implant Dentistry, Faculty of Dentistry, University of Seville.

Introduction: Immediate loading constitutes a clinical protocol that shortens the treatment period with good clinical results. The aim of this work is to present a clinical case with a comprehensive approach of the different implant techniques that constitute the immediate loading of dental implants in a geriatric patient.

Clinical Case: 60 years-old woman, with no medical history of interest. The clinical examination shows that the patient suffers from a chronic periodontal disease. In the anterior sector of the mandible, some teeth which are irreversibly affected with severe periodontitis. A plan of integral treatment is realized with the extraction of six teeth and the immediate insertion of 3 implants. In the same session, the impression is taken and an immediate fixed prosthesis is performed. After 6 months, the final fixed ceramic prosthesis is performed. The follow-up period was 3 years after the loading without surgical or prosthodontic complications.

Conclusions: The immediate loading of postextraction implants may constitute a clinical protocol that improves the healing of soft and hard tissues, improving the functional and aesthetic expectations of the geriatric patient.

\section{- Poster 19}

TITLE:- Maxillary supported-implant rehabilitation and extraction of retained canine in edentulous geriatric patient.

AUTHORS: Escuín Jimenez P, Moran Gutierrez R, Moreno Nieto M, Moreno Muñoz, Nuñez Márquez J, Velasco Ortega E.

Master of Implant Dentistry, Faculty of Dentistry, University of Seville.

Introduction: The surgery of the maxillary edentulous for implants requires an accurate diagnosis of the bone structures to perform an adequate assessment of the patient. The objective of this work is to present a clinical case with a comprehensive approach of the surgical and prosthetic implant techniques that constitute the rehabilitation of the total edentulous patient.

Clinical Case: A 66 years old woman with no medical history of interest. The clinical examination shows that the patient has maxillary total edentulism with a canine included. Treatment planning is performed with removal of the canine and placement of several implants for superior oral rehabilitation. After a reline of full denture is performed. At 6 months, the second surgery is performed with the placement of the healing screws and 15 days later the impressions are taken for a definitive fixed prosthesis ceramic cemented. The follow up period was 3 years after loading without surgical or prosthodontic complications.

Conclusions: The rehabilitation of the edentulous maxilla requires, sometimes, oral surgery techniques to prepare the maxilla in the insertion of implants. In these cases, bone regeneration techniques may also be necessary.

\section{- Poster 20}

TITLE:- Use of osteotomes in bone expansion and transalveolar elevation of the sinus in the geriatric patient with maxillary atrophy.

AUTHORS: Gfrorer Reina A, Jimenez Martin I, Lago Pacheco E, Moreno Muñoz, España Lopez A, Velasco Ortega E.

Master of Implant dentistry. Faculty of Dentistry. University of Seville.

Introduction: The etiology of maxillary bone resorption is multifactorial and influenced by tooth loss, diseases, and age. Thus, bone resorption continues after tooth extraction, producing a decrease in height and width. With the decrease in bone height, the remnant bone narrows and becomes closer to the nasal cavity, maxillary sinuses, and the group of nerves of the incisive canal. The possibility of inserting implants in the atrophic maxilla, in a non-traumatic way, has been possible by the introduction of suitable instruments such as osteotomes. The elevation of sinus floor by osteotomes, which was less invasive than procedures previously used for sinus floor elevation. In this approach, the Schneider's membrane and the floor of the maxillary sinus are elevated with osteotomes using a crestal approach, without preparation of a lateral window.

Conclusions: In general terms, the increase of the alveolar ridge through a corticotomy and posterior expansion of the ridge and transalveolar elevation sinus by osteotomes for the insertion of the implants has been suggested as an excellent geriatric implant technique.

\section{- Poster 21}

TITLE:- Maxillary overdentures with implants in geriatric edentulous patients.

AUTHORS: Garcia Navarro JR, Ruiz Garrido R, Salgado Peralvo O, Matos Garrido N, Ortiz García I, Velasco Ortega E.

Master of Implant Dentistry, Faculty of Dentistry, University of Seville. 
Objectives: Today, implant dentistry constitute a therapeutic modality in the prosthodontic treatment of totally edentulous patients. The aim of this study is to show scientific evidence of maxillary overdentures with implants.

Material and Methods: Electronic review on the Pubmed/Medline platform with the following keywords: dental implants, osseointegration, geriatric patients, edentulous patiens, maxillary overdentures. Subsequently apply the Spanish and English filters.

Results: Use of osseointegrated implants to restore edentulous maxilla has increased in the treatment of geriatric patients. Clinical dentistry have demonstrated the successfull application of implant dentistry techniques in older patients. Overdentures are an excellent treatment modality of implant prosthesis with a good functional and aesthetic response in these patients. Medical assessment and treatment planning is needed for optimize the benefits of oral rehabilitation with implant overdentures over quality of life in geriatric patients. Several implants can be retentive components, by attachments, of maxillary overdentures.

Conclusions: Clinical outcomes of studies demonstrate that prosthodontic rehabilitation in the maxilla of edentulous patients with overdentures supported by several implants is a successful implant treatment.

\section{- Poster 22}

TITLE:- Immediate loading with mandibular overdentures with miniimplants in geriatric edentulous patients.

\author{
AUTHORS: Perez Hernandez D, Baldrich Arenas \\ R, Cabanillas Balsera D, Jimenez Guerra A, España \\ Lopez A, Velasco Ortega E. \\ Master of Implant Dentistry, Faculty of Dentistry, University of \\ Seville.
}

Introduction: Implant dentistry constitute an important therapeutic modality in the prosthodontic treatment of geriatric patients with total tooth loss. This study reports the evaluation of edentulous patients treated with overdentures in the mandible by immediate loading of titanium miniimplants with sandblasted and acid-etched surface.

Material and Methods: Electronic review on the Pubmed/Medline platform with the following keywords: dental implants, geriatric patients, edentulous patient, mandibular overdentures, immediate loading. Subsequently apply the Spanish and English filters.

Results: Treatment planning included a radiographic diagnosis by panoramic o cone beam computarized tomography according the bone atrophy. Several implants are inserted, by flapless technique, in the interforaminal area, in one-stage surgery and are loaded immediately. The overdenture is perfomed with ball attachments retained with 4 implants.

Conclsuions: The revision of literature showed that prosthodontic rehabilitation in the mandible of edentulous patients with overdentures supported by titanium miniimplants can achieve osseointegration and were loaded immediately as a successful dental treatment.

\section{- Poster 23}

TITLE:- Guided bone regeneration in treatment with dental implants in the geriatric patient.

AUTHORS: Ramos Palenzuela M, Romero Reyes J, Minaya Tueros H, Nuñez Márquez E, Moreno Muñoz J, Velasco Ortega $\mathbf{E}$

Master of Implant Dentistry. Faculty of Dentistry. University of Seville.

Dental implants are the best treatment option for restoring the function of missing teeth. However, tooth loss can cause certain anatomical limitations that make implant placement difficult, such as maxillary sinus pneumatization and a lack of space between the alveolar bone and maxillary sinus. Elderly patients are one of the groups most affected by changes in both maxillaries; by a severe edentulism, as a result, they often need advanced surgery techniques in order to place dental implants. Guided bone regeneration procedures were first used in the 90s of XX century for place osseointegrated dental implants in atrophic maxilla and the presence of localized bone defects. The use of several types of membranes and biomaterials has improved the success of this regenerative technique. The following review of the literature was carried out to help shed light on recent developments in the field over the last 5 years.

\section{- Poster 24}

TITLE:- Effects of non-steroidal anti-inflammatory drugs in the osseointegration of dental implants in geriatric patients.

AUTHORS: Morán Gutiérrez R, Minaya Tueros H, Moreno Nieto M, Jiménez Guerra A, Ortiz Garcia I, Velasco Ortega E.

Master of Implant Dentistry, Faculty of Dentistry, University of Seville.

Objectives: Making a bibliographic review to evaluate if osseointegration processes in dental implants may be affected by the use of Non-steroidal Anti-inflammatory Agents (NSAIDs). 
Material and Methods: A bibliographic electronic search was performed using Pubmed and Medline databases, selecting articles published between March 1982 and February 2016. Keywords used for the search have been: "non-steroidal anti-inflammatory drugs," "dental implants", "geriatric patient" and "osseointegration". Selecting 14 articles according to the study.

Results: Nowadays, $83.1 \%$ of patients over 65 years of age take daily medication, which $14.1 \%$ take NSAIDs, of that total, $42.1 \%$ were occasional users, $24.9 \%$ acutely and $32.9 \%$ chronically. Studies show that chronically administered NSAIDs lead to unsuccessful results because COX-2 inhibitors damage in osseointegration, producing more negative effects. However, administration of a short course for post-operative pain management, not have a significant effect in said marginal peri-implant bone.

Conclusions: Patients who take NSAIDs chronically, for example patients with rheumatoid arthritis, may experience reduced success since the process of osseointegration can be affected. While the use of NSAIDs in a short period of time does not have a significant effect on periimplant marginal bone during their healing period, so there is no risk of affectation in the osseointegration.

\section{- Poster 25}

TITLE:- Maxillary sinus lift technique with the use of bone graft in geriatric patients.

AUTHORS: Arenas Baldrich R, Fernandez Garcia N, Garcia Tirado A, España López A, Nuñez Márquez E, Velasco Ortega E.

Master of Implant Dentistry. Faculty of Dentistry. University of Seville.

Introduction: A fundamental prerequisite for implant placement is the presence of an adequate quantity of bone. The aim of this study is to show the sinus floor elevation technique in edentulous geriatric patients.

Material and Methods: Electronic review on the Pubmed/Medline platform with the following keywords: dental implants, geriatric patients, edentulous maxilla, sinus floor elevation, biomaterials. Subsequently apply the Spanish and English filters.

Results: The posterior edentulous maxilla often shows insufficient bone volume, which represents a great challenge for the surgeon. As a matter of principle, this technique involves the elevation of the sinus membrane from the floor of the maxillary sinus to allow the placement of bone graft. Different grafting materials have been proposed for sinus augmentation. Autogenous bone graft was considered the gold standard thanks to the bone morphogenetic proteins and other growth factors that confer unique osteogenic, osteoinductive and osteoconductive properties. However, harvesting from an intraoral or extraoral site requires a second surgery, increasing the duration of the procedure and the morbidity of the patient. To prevent such disadvantages, other materials have been advocated to simplify the grafting procedure, furthermore reducing surgical time and patient discomfort.

Conclusions: Allografts, xenografts and alloplastic materials are characterized by osteoconductive properties, providing an essential scaffold for ideal bone growth without the need for a second surgical procedure. The most frequent complications of this surgery are the Schneider membrane perforation, sinusitis, ostium obstruction or mucosal dehiscence.

\section{- Poster 26}

\section{TITLE:- Implant loading protocols in elderly} patients.

AUTHORS: Fernández García Nuria, Gforer Reina A, Haidar Wehbe A, España Lopez A, Nuñez Márquez E, Velasco Ortega E.

Master of Implant Dentistry. Faculty of Dentistry. University of Seville.

Introduction: Oral implantology is an important therapeutic modality in the prosthodontic treatment of older patients with tooth loss. The knowledge of the biological phenomena of the osseointegration and the good results of the research have perfomed the development of implant loading protocols.

Material and Methods: A paper review has been carried out in the database Pubmed and Medline between the years 2004 and 2016.

Results: Functional loading of osseointegrated implants at 3-6 months of insertion has been considered for years as a sine qua non condition for achieving and maintaining osseointegration. Studies of early and immediate loading inserted into the mandible of geriatric patients provide good clinical results with excellent osseointegration. Immediate loading protocols allow the existence of certain micromovements that do not appear to affect a favorable bone tissue response.

Conclusions: A comprehensive diagnostic evaluation of geriatric patient and a correct planning by of a suitable surgical and prosthetic protocol allows to obtain an alternative of success in the treatment.

\section{- Poster 27}

TITLE:- Prosthetic complications in two implant-supported overdenture treatment in geriatric mandibular edentulous patients. 
AUTHORS: España Guerrero E, Martí Villaescusa A, Mesa Sánchez $C$, Moreno Muñoz J, Nuñez Márquez E, Velasco Ortega E.

Master of Implant Dentistry. Faculty of Dentistry. University of Seville.

Introduction: Mandibular two implant-supported overdenture is one of the most used treatments in edentulous elderly patients for surgical, aesthetic and economic reasons.The aim of this work is to show clinical outcomes of this technique in geriatric mandibular edentulous patients.

Material and Methods: Elderly patients with total mandibular edentulism were treated with 2 anterior implants for overdenture rehabilitation. At 2 months prosthetic rehabilitation was carried out using ball attachments or Locator ${ }^{\circledR}$ attachments. The clinical follow-up period has been at least 60 months after the functional load.

Results: 45 elderly patients were treated with $90 \mathrm{im}$ plants. Clinical findings demonstrate the excellent clinical response of this type of protocol. Results indicate a success rate of the implants of $100 \%$. After a 70 -month average period of functional loading since definitive rehabilitation, there have been no late surgical complications. The most important prosthetic complication was the fracture of the prosthesis by the retentive element to the implant in acrylic prostheses made, mostly ring systems. In prostheses retained by locator the most frequent complication was the removal of the metallic element from the base of the prosthesis, followed by the wear of the locator abutment. Other technical complications were the wear of the elastomer ring on the ball attachments. Relining of the denture contribute to the marginal sealing and to maintain the mucous-implant support, avoiding overloads to the prosthesis.

Conclusions: This study indicates that the treatment with two dental implants in symphysis for overdenture rehabilitation in the mandible is an implant technique with a high rate of surgical success and with easy solution of prosthetic complications.

\section{- Poster 28}

\section{TITLE:- Risks of placing dental implants in ge- riatric patients taking bisphosphonates.}

AUTHORS: Minaya Tueros H, Garcia Navarro JR, Garcia Sánchez A, Moreno Muñoz J, Nuñez Márquez E, Velasco Ortega E.

Master of Implant Dentistry. Faculty of Dentistry. University of Seville.

Introduction: Bisphosphonates are a group of drugs that are commonly used to alter bone metabolism in order to prevent bone loss in diseases such as osteoporo- sis and bone cancers. This update will present the latest evidence and guidelines available on bisphosphonates and their possible effects on implant dentistry.

Material and Methods: Electronic review on the Pubmed/Medline platform with the following keywords: dental implants, osseointegration, geriatric patients, bisphosphonates, osteonecrosis. Subsequently apply the Spanish and English filters.

Results: The use of bisphosphonates has been associated with osteonecrosis of the jaws. The debate as to whether it is wise to consider placing dental implants in patients being treated with bisphosphonate therapy remains a controversial area. The risk factors, co-morbidities, clinical outcomes and findings from various modalities for bisphosphonate-related osteonecrosis of the jaws are highlighted. The management of patients being treated with bisphosphonates, in whom dental implants might be considered or have already been placed, will also be discussed.

Conclusions: The use of bisphosphonates must be considered in the treatment with dental implants in geriatric patients with osteoporosis.

\section{- Poster 29}

TITLE:- Bone expansion in severe reabsorbed crests for the insertion of dental implants in geriatric patients.

AUTHORS: Lago Pacheco E, Perez Hernandez D, Ramos Palenzuela M, España Lopez A, Jiménez Guerra A, Velasco Ortega E.

Master of Implant Dentistry. Faculty of Dentistry. University of Seville.

Introduction: Bibliographical review of articles published in the last 10 years on the placement of implants in crests with advanced resorption, making it necessary for their placement the use of different expansion techniques.

Material and Methods: Electronic review on the PUBMED / MEDLINE platform with the following keywords: split crest, bone expansion, edentulous ridge explansion, dental implants. Subsequently apply the Spanish and English filters.

Results and Discussion: Age is directly related to tooth loss, increasing the odds of partial or complete edentulism. The absence of the dental stimulus on the bone surface causes a loss of volume both in height and width, making it necessary to expand the bony rim for rehabilitation with implants.

Conclusions: After the long-term follow-up of the implants placed by means of the expansion techniques, a success rate above $90 \%$ is referred, reason why it is a viable therapeutic alternative for the patient. 


\section{- Poster 30}

TITLE:- Surgical protocol of anticoagulated elderly patient in the treatment with dental implants.

AUTHORS: Mesa Sánchez C, Lago Pacheco E, Romero Reyes J, Jiménez Guerra A, España Lopez A, Velasco Ortega E.

Master of Implant Dentistry. Faculty of Dentistry. University of Seville.

Objectives: Given the high incidence of anticoagulated elderly patients in dental clinics and the increase in surgical procedures, the aim of this systematic review is to assess the risks of uncontrolled bleeding if the medication is continued against the possibility of thromboembolic complications if discontinued.

Material and Methods: PubMed database was searched for articles with the following keywords: "oral surgery", "extraction", "anticoagulants", "warfarine", "post-operative bleeding". Subsequently, the English, Spanish, and human filters were applied.

Results: No significant difference was found comparing postoperative bleeding in patients who had interrupted anticoagulant therapy and patients who had not in the numerous clinical cases and existing studies. In some of this cases it was recommended to consult the INR before surgery, and the application of local hemostatic measures at the end.

Conclusions: Oral surgeries can be safely performed for patients on anticoagulant therapy as long as the INR level is maintained $\leq 3.0$ and effective local hemostasis measures is given.

\section{- Poster 31}

TITLE:- Bisphosphonate therapy in the treatment with dental implants in older patients.

AUTHORS: Moreno Nieto M, Ruiz Garrido R, Salgado Peralvo O, Ortiz Garcia I, Matos Garrido N, Velasco Ortega E.

Master of Implant Dentistry. Faculty of Dentistry. University of Seville.

Introduction: Today, it is very common that geriatric patients who require treatment with dental implants have a bisphosphonate therapy. In this literature review we will evaluate the osseointegration of the dental implant in patients with bisphosphonate therapy and the risk of osteonecrosis of the jaws after the surgical procedure.

Material and Methods: Electronic review on the Pubmed/Medline platform with the following key- words: dental implants, dental implants, osseointegration, geriatric patients, bisphosphonates, osteonecrosis. Subsequently apply the Spanish and English filters.

Results: Moreover, we will indicate clinical considerations we must consider in these patients so that complications are predictable and our implant treatment is satisfactory for both of them, the clinician and the patient. Conclusions: Bisphosphonates treatment is not a contraindication for dental implants, because it does not decrease osseointegration of the dental implant, although it is true that there are cases of osteonecrosis of the jaws in patients with bisphosphonate therapy.

\section{- Poster 32}

TITLE:- Medical assessment and treatment planning from a comprehensive implant approach of the geriatric patient.

AUTHORS: García Tirado A, Martí Villaescusa A, Jiménez Martin I, Moreno Muñoz J, Nuñez Márquez E, Velasco Ortega E.

Master of Implant Dentistry. Faculty of Dentistry. University of Seville.

Introduction: During last decades, implant dentistry has been an increased technique in geriatric dentistry. The aim of this study is to review the role of the dentist in restoring a healthy oral status and increasing quality of life of these people through a multidisciplinary treatment combining periodontal, conservative, endodontic and implant prosthetic therapies.

Material and Methods:.Electronic review on the Pubmed/Medline platform with the following keywords: dental implants, implantology, geriatric patients, comprehensive approach, interdisciplinary dental treatment. Subsequently apply the Spanish and English filters.

Results: From a comprehensive approach, is necessary a medical assessment of the older patient, an oral diagnosis, and a correct treatment planning, with a strict protocol of surgical, restorative and prosthetic procedures. In many older patients, their overall medical condition may be complex and must be assessed for implant surgery. After the surgical step, a good reaction of hard and soft tissues is necessary for long-term success of dental implants. Periodontal and restorative treatment are very important for the remanent teeth. Fixed and removable prostheses supported by dental implants were been indicated in edentulous older patients, according medical functional evaluation.

Conclusions: Maintenance by the patient and the dentist are very important for the success of dental treatment. Implant-supported prostheses improve oral quality of life and psychosocial well-being of edentulous older patients. 


\section{- Poster 33}

TITLE:- Satisfacion of older patients by the use of two implant-retained mandibular overdentures.

AUTHORS: Ruiz Escolano MG, España López A, Jiménez Guerra A, Ortiz García I, Matos Garrido N, Velasco Ortega E.

Master of Implant Dentistry. Faculty of Dentistry. University of Seville.

Introduction: Mandibular implant-retained overdenture by two anterior implants is one of the treatments of choice for elderly total mandibular edentulous patients. The aim of this study is to show the satisfaction level of geriatric patients treated with mandibular overdentures.

Material and Methods: A oral health quality survey for assessing the degree of satisfaction of 55 elderly patients carrying two implant-supported mandibular overdentures has been carried out.

Results and Discussion: All patients were quite satisfied and they did not request any additional treatment with a higher number of implants. The possibility of a direct bonding of the prosthesis to the osseointegrated implants, and the transmission of forces from the artificial teeth to the mandible ensures the prosthesis' stability, decreasing oral soft tissue trauma, improving the quality of life of elderly patients. It was stressed that the maintenance needed by the prostheses on the implants was greater than in normal complete prostheses. The satisfactory results of the different systems have been documented with a good long-term prognosis, what allows to recommend its use in elderly mandibular edentulous patients.

Conclusions: Mandibular implant-supported overdenture in elderly patients is one of the best therapeutic options of prosthesis on implants with a good biological and functional response in these patients.

\section{- Poster 34}

TITLE:- Survival of implants in the rehabilitation of patients subject to mandibular research surgery by COCE. Clinical case.

\footnotetext{
AUTHORS: Laime Rojas CS, Garay García AL, Castañeda Vega PM, López López J, Jané Salas E.

Master's degree in dentistry in cancer patients and immunocompromised patients. Faculty of Medicine and Health Sciences, UFR Dentistry, Bellvitge Campus, Barcelona Spain.
}

Introduction: Oral COCE squamous cell carcinoma is the most frequent oral carcinomas representing ap- proximately $3-4 \%$ of malignant neoplasms. It has been associated over 60 consumers of tobacco and alcohol but is currently observed an increase in young men in which risk factors are not present (HPV). The resection surgical of carcinoma squamous oral entails sequels, the patient is will find with alterations functions as chewing, phonation, swallowing, of there the challenge for the surgeons of replace them structures operated.

Clinical Case: On the Dental Hospital University of Barcelona will visit a 60 year-old patient with a medical history of vitiligo, thyroidectomy, and resection of COCE in the floor of the mouth without radiation seeking rehabilitation. Assessing the amount of fabric away and degree of mandibular condition individually, determines the rehabilitation with implants Strauman Standard Plus 4.8 WN x 8mm / Bone Level 4.1 RC x $12 \mathrm{~mm}$ in edentulous areas, is reinstated missing teeth achieving great functional and aesthetic improvement. The rate of success in osseointegration of implants in patients oncological subjected to resections surgical are of $92.9 \%$ and a load failed of $15 \%$ (osseointegration or bad positioning) in a period of 3 years, the failure affects particularly to the Group of patients irradiated.

Conclusions: Rehabilitation with implants in cancer patients achieve long-term success, currently treating implant - prosthetic is a reliable technique that has proven high survival and osseointegration.

\section{- Poster 35}

TITLE:- Review of laser types for the treatment of oral leukoplakia due to a clinical case.

\footnotetext{
AUTHORS: Valls Roca Umbert J, Blazquez Hinarejos M, López López J, Jané Salas E.

Máster en Medicina, Cirugía e Implantología Oral. Facultad de Medicina y Ciencias de la Salud, Odontología. Universidad de Barcelona.
}

Introduction: We received at our master a 72 year old smoker patient, with a leukoplakia lesion in the palate and the corner of the lip. We performed a biopsy and sent it for the anatomopatological study.

Objectives: Know if the laser treatment is the best way to treat the lesion.

Material and Methods: Performed a review of the articles about laser treatment for leukoplakia published the last 8 years. Using keywords: Laser and oral leukoplakia, laser types for oral leukoplakia, oral leukoplakia treatment in PubMed search engine. We consulted the book Medicina y Patología Oral (Bagán).

Conclusions and Results: Many authors coincided that when treating a leukoplakia the first step to undertake is to eliminate the factors that could cause the appearance of the lesion or its malignitzation. When certain 
criteria of malignitzation appear, such as dysplasia, the surgical treatment becomes the best course of action. Within the surgical treatments to eliminate the lesion, some authors point out that the treatment with laser is the most effective, since in most cases it will provide a complete removal of the lesion, with minimal pain, intra and post operational complications and a lower degree of reappearance. The mentioned lasers would include $\mathrm{CO}_{2}$, Potassium Titanyl Phosphate (KTP) and Neodymium-Doped Yttrium Aluminium Garnet (YAG), several studies mention that the KTP laser appears to have less reappearance of the lesion after treatment than the $\mathrm{CO}_{2}$ and YAG due to its properties.

\section{- Poster 36}

\section{TITLE:- Revision of preprosthetic frenectomy in elderly patients.}

AUTHORS: Domínguez Mínger J, Roca Millán E, Morera García J, Estrugo Devesa A, Jané Salas E, López-López J.

Department of Odontostomatology, Faculty of Medicine and Health Sciences (Dentistry). University of Barcelona.

Aim: A revision of the importance of the preprosthetics frenectomy, and the most recommended surgical technique in the elderly patients.

Material and Methods: A bibliographic electronic research in the data bases "PubMed", "Scielo" and "Cpchrane" was performed with de key words "preprosthetic surgery", "frenectomy complete dentures", following the inclusion criteria: full text available, English or Spanish language, focusing on the importance of frenum in prostheses and the most recommended surgical techniques for frenectomy.

Results: From a total of 235 references we have finally selected 11 articles: 5 reviews, 3 clinical trials and 3 case reports, about frenectomies carried out in patients over 65 years old following different techniques and the advantages and indications of this intervention

Conclusions: The importance of pre-prosthetic frenectomy lies in a mucosal prosthesis with better retention, a lower risk of ulceration in the areas of continuous trauma and a lower risk of fracture.

The technique of z-plasty with scalpel is a good technique recommended for frenectomy that guarantees a migration of the frenulum insertion and an increase in the depth of vestibule.

The laser technique has shown advantages over conventional surgery with scalpel such as hemostasis and lower risk of postoperative infection, but more clinical trials are needed in older patients.

Taking into account the economic status of older patients, we should not underestimate the surgical proce- dures that we can carry out to ensure the correct functionality of a conventional complete prosthesis.

\section{- Poster 37}

\section{TITLE:- Atypical location of a lobular capillary angioma. A case report.}

\author{
AUTHORS: Maynau González A; Príncep C; Jané \\ Salas E; Estrugo Devesa A; Marí Roig A; López Ló- \\ pez J. \\ Máster en Medicina, Cirugía e Implantología Oral. Facultad de Me- \\ dicina y Ciencias de la Salud, Odontología. Universidad de Barce- \\ lona.
}

Introduction: Lobular capillary angioma is a relatively common non-neoplasic soft tissue tumor where there is a fibrovascular tissue development with endothelial proliferation. It can be produced by a local reaction to an irritant. It has received several names: pyogenic granuloma, botriomicoma, telangiectasic among others. It is more common in women than in men $(2: 1)$. The presentation age is very variable, although most cases are recorded between the second and fifth decades of life. The preferred localization is the interdental papilla $(70 \%$ of the cases), followed by the tongue.

It appears like a reddish, bluish or purple, soft, sessile or pediculated tumor, its size can vary from a few millimeters to several centimeters. The diagnosis is clinical and histological; and the treatment is surgical excision executed with caution taking into consideration the intra-operative and post-operative bleeding, making a well curettage of the entire area underlying the insertion to avoid recurrences.

Case Report: A 71-year-old male patient reported to the Master of Medicine, Surgery and Oral Implantology of the University of Barcelona due to a lesion in the floor of the mouth, hyperplasic at the level of teeth 32-33 with a size of $12 \times 10 \times 3 \mathrm{~mm}, 4$ months of evolution and soft consistency . Periapical radiographs showed slight bony involvement of the alveolar ridge: The diagnosis of a giant cell peripheral tumor was suspected. The excisional biopsy was performed and the histopathological diagnosis indicated the presence of a lobular capillary angioma; the floor of the mouth being an infrequent location.

Conclusions: It is concluded that the pathological anatomy diagnosis is essential for the definitive diagnosis of this type of lesions, since we cannot be guided only by the most frequent locations described in the literature. Regardless of its benign nature, the proper diagnosis of lobular capillary angioma is always clinically important and its subsequent treatment. 


\section{- Poster 38}

TITLE:- Implant prosthetic rehabilitation of a patient with a medical history of OSCC. A case report.

AUTHORS: Schemel Suárez M, Pérez Losada F, Castañeda Martin P, Estrugo Devesa A, López López J, Jané Salas E.

Master of Dentistry on Oncologic and Immunocompromised $\mathrm{Pa}$ tients. Faculty of Medicine and Health Sciences, UFR Dentistry, Campus Bellvitge, Barcelona, Spain.

Introduction: Prosthetic rehabilitation on patients who have undergone treatment for head and neck cancer is sometimes difficult because of the functional and esthetic sequelae. Treatment plan must be completely individualized in order to satisfy the patient's needs. The aim of this study is to present the treatment plan performed on a woman with a medical history of oral cancer and completely edentulous maxillae.

Case Report: A 70 years old woman, with a history of oral squamous cell carcinoma on the lateral left side of the tongue surgically treated with a partial mandibulectomy and neck dissection; comes to the dental clinic for a consultation after several months have passed from the oral surgery; her main concern was the rehabilitation of her teeth. We decided to make an upper implant-supported fixed denture because a complete conventional denture would have been unstable. The implants placement was performed with guided surgery and the prosthetic rehabilitation was completed with CAD-CAM technology. Conclusions: Dental implants placement and prosthetic rehabilitation on individuals with medical history of head and neck cancer is a good and predictable treatment alternative, taking into consideration that a periodic follow up of the patient is necessary.

\section{- Poster 39}

TITLE:- Antihypertensivs drugs: Do they improve osseointegration?

AUTHORS: Sáez Alcaide LM, González Serrano J, Molinero Mourelle P, González Fernández Tresguerres F, Paredes Rodríguez VM, López Quiles Martínez J.

Objectives: Estimations suggest more than $65 \%$ of the population over 60 years old suffer from hypertension, and more than half of them are receiving antihypertensive drugs. The aim of this study is to evaluate the influence of these drugs in patients treated with osseointegrated implants.

Material and Methods: A literature review was con- ducted over articles published within the last ten years with the keywords "antihypertensive medications" AND "antihypertensive drugs" AND "osseointegrated dental implants" AND "implant treatment".

Results: The study suggests antihypertensive drugs have a positive impact on bone metabolism. This happens through different pathways such as inhibition of osteoclast activity or renal calcium excretion.

Conclusions: Antihypertensive drugs increase the medium and long-term success rate of the treatment with implants because of their protective role on bone metabolism.

\section{- Poster 40}

TITLE:- Salival biomarkers for Alzheimer disease.

AUTHORS: Salgado González C, Diez Dans G, Rigesti Fertonani M, Martín Carreras Presas C, Paredes Rodríguez VM, Hernández Vallejo G.

Titulo Propio de Especialista en Medicina Oral. Facultad de Odontología. Universidad Complutense de Madrid.

Introduction: Alzheimer's disease (AD) is a progressive neurodegenerative disorder with distinctive clinical and pathological features, although there are individual differences in the age of onset and pattern of cognitive impairment. It is estimated that dementia currently affects more than 24 million people, a cipher that will duplicate every 20 years to exceed 80 million of affected people by 2040 .

Objectives: To identify salivary biomarkers for the diagnosis and monitoring of Alzheimer's disease.

Material and Methods:: A literature review was performed using Pubmed and Medline databases. Key words: oral biomarkers, Alzheimer, saliva as diagnostic fluid.

Conclusions: The diagnosis of Alzheimer's disease (AD) is currently based on clinical evaluations, psychological and imaging tests. These processes are expensive and don't allow performing an early diagnosis of AD. At the time of diagnosis there is already a significant deterioration in the patient.

Biomarkers are presented in the near future as a reliable alternative because, they are inexpensive, easily reproducible, non-invasive and reliable. We are going to provide an early diagnosis of the disease, allowing us to advance the treatment and slowing down the deterioration of the quality of life of these patients.

\section{- Poster 41}

TITLE:- Recurrent traumatic ulcer.

AUTHORS: Olivera Oliva A., Jané Salas E., García Ramón S., Estrugo Devesa A., Mari Roig A., López López J. 
Máster en Medicina, Cirugía e Implantología Oral. Facultad de Medicina y Ciencias de la Salud, Odontología. Universidad de Barcelona.

Introduction: The oral injuries in elders are very frequent and constitute a health-related issue, being the most frequent mouth sores. It is considered traumatic chronic ulceration a loss of substance from outside to inwards, depth and variable size and shape rounded or oval. The reason obeys any mechanical agent which acts in constant form. The xerostomia, the medication and the psychological factors could influence in his onset. The traumatic sores can repair at several hours if the reason is suppressed. But if the reason persists, in chronic form, the characteristics of the injury are modified.

Clinical Case: 72-year-old patient woman, with precedents of rheumatoid arthritis and polymedicated, she comes to our service for presenting repetition sore in the lateral left edge of the tongue since summer of 2015. With clinic and variable symptomatology. In the intraoral exploration, she presents dry mouth in treatment and a sore injury, painful of $2 \times 2 \mathrm{~mm}$. We decide to request a control analytical, analyze the parafunctional habits, clean the crowns and make controls every three months to observe evolution and realize a biopsy.

Conclusion: In this type of injuries is necessary to search the reason to eliminate and control his evolution. It is important to take into account the parafunctional habits of the patient, the tobacco, the mechanical traumatism, the mouth sepsis, the general diseases like diabetes, circulatory problems, neurological disorders or medicaments, especially in geriatric patients, to prevent that these injuries could turn in precancerous.

\section{- Poster 42}

\section{TITLE:- Statins use in dentistry.}

\author{
AUTHORS: Pucci F, Govani A, Awan N, Martín \\ Carreras Presas C, Somacarrera Pérez ML, Gomez \\ Font R. \\ Faculty of Biomedical Sciences. Adult's Dentistry Department. Eu- \\ ropean University of Madrid.
}

Summary: Statins are the best known drugs used in LDL-C cholesterol control on high cardiovascular risk patients. They act by inhibiting HMG-CoA enzyme which control cholesterol synthesis via Mevalonato pathway. Recent studies show that statins posess pleiotropic effects which could positively affect bone regeneration.

Objectives: The purpose of our work is to carry out a bibliographic revision to study the influence of statins in bone regeneration and to clarify its possible use in dental practice.
Material and Methods:: We made a bibliographic revision of the existing scientific literature, using databases such as Medline and Pubmed from Dulce Chacon library belonging to the UE.

Key words: Statins, Bone regeneration, Statin's pleiotropic effect.

Results: Statins boost the activation and production of bone tissue through the stimulation of mRNA increasing the expression of Bone Morphologic Protein (BMP-2), Vascular Endothelial Growth Factor (VEGF), alkaline phosphatase, Type 1 collagen and osteocalcin. Studies investigated the effects of local statin application to take advantage its pleiotropic effect in pathologies such as chronic periodontitis, implants osseointegration and filling of isolated bone defects.

Conclusions: We need more studies to determine the possible long-term effect of Statins in regenerative dental practices.

\section{- Poster 43} TITLE:- Cholesterol levels and bone regenera-
tion in third age patients.

AUTHORS: Formini E, Almazán Martínez S, Viehmann M, Gómez Font R, Somacarrera Pérez ML, David Ubiñas K.

Facultad de Ciencias Biomédicas. Departamento de Odontología del adulto. Universidad Europea de Madrid.

Summary: Dyslipidemia and other disorders may increase cholesterol levels, which may affect the process of bone regeneration in the third age patients.

Objectives: We analyse aspects such as the age of the patient as a risk factor in the onset of systemic diseases, factors involved in bone formation and certain drugs involved in cholesterol control, in particular the correlation between statins and bone regeneration .

Material and Methods: We review scientific papers published between 2005 and 2017 with the keywords: Colesterol, Estatinas, Regeneracion osea, dislipemia, pacientes de tercera edad, third age patient, bone regeneration, cholesterol.

Results: It is evaluated how cholesterol levels influence the bone metabolism affecting regeneration in geriatric patients.

Conclusions: Research suggests that cholesterol influences bone metabolism by inducing, at high levels, cellular apoptosis, especially if there is excess intracellular LDL. In addition, it has been observed how the statins improve the control of cholesterol and favor cell reproduction, having a positive effect on the bone regeneration of the elderly patients. 


\section{- Poster 44}

TITLE:- Implant-prosthetic rehabilitation in the edentulous patient.

AUTHORS: Anastasio MD, Almazán Martínez S, Viehman M, Govani A, Gómez Font R, David Ubiñas KM

Faculty of Biomedical Sciences. Department of Adult's Dentistry. European University of Madrid.

Introduction: The functional and aesthetic implantprosthetic rehabilitation of the edentulous patient can be developed in three options: total prosthesis, overdentures and all-on-four.

Objectives: To analyze the prognosis, advantages and disadvantages of the implantoprotesic treatment in relation to the aggression that it supposes for an elderly edentulous patient, in front of less aggressive treatments.

Material and Methods: We have carried out a bibliographic search in pubmed, medline, google academic of the last five years, with the key words: implantoprotesic rehabilitation, overdentures, implants in geriatric patients, all-on-four, complete prosthesis.

Results: There are treatments for the restoration of edentulism in elderly patients, which don't compromise the aesthetic, functional results nor the postoperative health status of the patients. The all-on-four is a technique currently applied, it is usually indicated in large resorptions, having enough prothetic space. Total prostheses are usually of choice especially in some cases in which the patient's health doesn't allow implant placement or when the economic factor is very relevant for the patient.

Conclusions: Many authors say that all-on-four has a survival rate of $92.2 \%-100 \%$. Treatments with fewer implants mean less aggression for the elderly patient. Non-aggressive treatments are preferred by a large number of patients and professionals.

\section{- Poster 45}

\section{TITLE:- Relationship between depression and oral lichen planus.}

AUTHORS: Mansilla Romaní M, Salgado González C, Díez Dans G, Piñas Alonzo R, De Arriba De La Fuente L, Hernández Vallejo G.

Postgrate of Specialist in Oral Medicine. Faculty of Dentistry. Complutense Unviersity of Madrid.

Introduction: Major depression is a psychiatric disorder wich occurs in adults since the third decade of life. Oral liech planus (OLP) is a chronic inflammatory disease, non infectious, mucocutaneous involvement and autoinmune etiology that appears between the third and sixth decade. Despite an unclear etiopathogenesis, there have been reported psychologically factores like anxiety, stress and depression as posible triggers.

Material and Methods: A bibliographic review of the last ten years was med using as databases Pubmed and Medline. Fort he search criteria, keywords such as "oral lichen planus", "elderly" and "dperession" were used. In total, fourteen articles were selected, six review articles, five cases and controls articles, two prospective studies and one retrospective study.

Results: There are many studies that reveal the existence of a relationship between patiens with OLP and depression. Some of these articles link symptomatic OLP cases with more severe and prolonged depression stages, while others articles affirm that during the deprressive symptoms exists more exacerbations of the OLP.

Conclusions: In patients with OLP and depression, a bidirectional relation can be established in the evolution of both diseases. Due to the role that the psychological component can play, it is necessary to consider psychotherapy as a complementary treatment in cases of symptomatic OLP.

In conclussion, in elderly patients where mucocutaneous, psychiatric and immune system disorders can happen at the same time, a multidisciplinary tretament is neccesary.

\section{- Poster 46}

TITLE:- Age as a risk factor for peri-implantitis in geriatric patients.

AUTHORS: Viehmann M, Anastasio D, Pucci F, Martín Carreras-Presas C, Herranz Calzada A, Gómez Font R.

Faculty of Biomedical Sciences. Adult's Dentistry Department. European University of Madrid.

Introduction: Due to the increase in the demand for implants, biological complications such as periimplantitis became increasingly frequent and are expected to continue to grow in the future.

Objectives:. To analyze the existing scientific evidence in the literature in order to establish the age from which the probability of peri-implantitis increases, the risk factors and the necessary preventive measures.

Material and Methods: A literature of the last 10 years was performed in PubMed Medline and Google Scholar, using the keywords: periimplantitis risk factors, periimplantitis diabetes, periimplantitis systematic review, periimplantitis etiology, periimplantitis prevalence.

Results and Discussion: Several studies identify as possible risk factor for peri-implantitis: diabetes, car- 
diovascular diseases, smoking and mainly periodontitis. Residual pockets, especially in cases of aggressive periodontitis, facilitate the appearance of peri-implant disease. Due to an increased presence of chronic diseases and an oral hygiene deficit, elderly patients are at greater risk of peri-implant complications. Therefore prevention, based on maintenance visits determined by oral hygiene, is essential since long-term treatments are unpredictable.

Conclusions: While there is more evidence on the relationship between peri-implantitis and periodontitis, along with an increased susceptibility with aging, other factors such as smoking, diabetes and cardiovascular diseases are controversial. Further prospective studies are needed to elucidate the etiopathogenic factors and progression of peri-implantitis. Maintenance visits and periodic reviews together with good oral hygiene seem to play an important role in its prevention.

\section{- Poster 47}

TITLE:- Comparison of sinus elevation techniques in geriatric patients.

AUTHORS: Herranz Calzada A, Viehmann M, Awan N, Martin Carreras Persas C, Somacarrera ML,Gómez Font R,

Biomedic Science school. Dentistry department. European University of Madrid.

Introduction: In elderly patients, a very small amount of bone is present below the maxillary sinus, requiring special techniques for implants and prothesis restorations. Mainly they are two techniques: crestal or closed and the lateral or open window, with multiple variations.

Objectives: To analyze the grade of aggression and morbidity of these techniques, as well as their results and predictability, in elderly patients

Material and Methods: We review the publications of the last 20 years, with a minimum sample of $40 \mathrm{im}$ plants in patients over 60 years old, with absence of basic pathologies.

Results: We found a greater need for initial bone in the open versus closed technique with a lower amount of elevated sinus.

Regardless of the technique, the main complications will be: perforation of the Schneider's membrane (minor with piezoelectric surgery), excessive bleeding (alveolar-transverse arteries) or post-surgical sinusitis. However, there are fewer complications in atraumatic elevation, compared to the side window (7-35\%) that has worse postoperative.

Patient satisfaction in both techniques is similar (95\%).
Conclusions: Each technique has its applications and we should look more at the patient's factors (bone quantity-quality, anatomy, type of graft used and pathologies of the patient) than in the technique.

The grade of success and satisfaction are similar in both techniques although the postoperative is worse in the open technique.

\section{- Poster 48}

\section{TITLE:- The use of the evaluation platform for prosthesis quality control in patients over 65 years.}

\section{AUTHORS: Leco Berrocal MI, Gutiérrez Cárdenas L, Barbero Fernández L , Serrano Granger C, Mar- tín Carreras Presas C.}

Faculty of Biomedical Sciences. Adult's Dentistry Department. Undergraduate Dental Office of The European University of Madrid.

Introduction: The Platform for the Evaluation of Dentistry Practices in the Dental Office of the European University of Madrid has become an essential tool in the monitoring and control of the treatments performed by our students. This has led to changes in the care of our patients, in terms of timing and quality of the treatments perfomed, which we consider that can affect the geriatric population, that comes to our University Dental Office demanding removable prosthesis.

Objectives: The main goal of our research work was to know if the implantation of the new Platform of Evaluation of Practices improves the attention of the geriatric patients susceptible of treatments with removable prostheses.

Material and Methods: We surveyed our faculties (38) and 5th year dental students (216) about work habits, feedback and wether the new platform was useful in order to guide them and thus facilitating the realization of the dentures and shortening prosthetic appointments in patients over 65 years old.

Results: The results of our surveys show that both teachers and students agree that the implementation of the evaluation platform served to improve the care of our geriatric patients. Counting on the clinical guidelines to be followed in each of the steps in the realization of the removable prosthesis contributes to the success of the treatment, minimizing the possible mistakes of the students.

Conclusions: Through the use of the Evaluation Platform, our students are able to integrate the acquired knowledge, which has a positive impact on the quality of the treatment, and thus on the satisfaction of our patients. 


\section{- Poster 49}

TITLE:-Oral treatment with general anesthesia in the elderly patient with cognitive disabilities.

AUTHORS: Cortezo Vidal V, Paredes Rodriguez VM, Gonzalez Serrano J, Garcia-riart Monzon M, Saez Alcaide LM, Martin Lopez FM.

Hospital General Universitario Gregorio Marañón.

Objectives: The purpose of this study is to establish the needs of attention of a population over 65 years old with cognitive disabilities that cannot receive regular treatment in a dental clinic with local anesthesia.

Material and Methods: 55 patients over 65 years of age with cognitive disabilities treated at the Gregorio Marañon University Hospital. The medical history was developed with the help of parents and caregivers and the treatment took place at the dental clinic or at the operating room depending on the predisposition of the patient. The treatments consisted on surgical treatments, fillings and cleanings. The statistical analysis was made with Chi cuadrado and the Fisher test.

Results: The main etiologies were Alzheimer's disease $(\mathrm{n}=28)$, Parkinson's disease $(\mathrm{n}=18)$ and brain strokes (9). The most common treatments were tooth extractions $(28,4 \%)$ followed by cleanings (25\%), soft tissue surgical procedures, and cavities (14\%). There is a higher presence of habits such as bruxism. Nearly half of the patients did not cooperate in the exploration tests or the X-rays.

Conclusions: The damage on the oral health of the elderly patient with cognitive disabilities is more severe due to their inability to cooperate on their dental higiene or during treatment at the dental clinic as well as due to the habits associated with their disease.

\section{- Poster 50}

TITLE:- Squamous odontogenic tumor. Systematic review and case report.

AUTHORS: Calvo Arcones E, Izquierdo Gómez K, Cuesta González F, González Navarro B, Jané Salas E, López López J.

Máster en Medicina, Cirugía e Implantología Oral. Facultad de Medicina y Ciencias de la Salud, Odontología. Universidad de Barcelona.

Introduction: The squamous odontogenic tumor (SOT) was described for the first time in 1975, since it has been described as a rare benign neoplasm.

Description of the clinical case:

Patient cames for extraction of a root remainder (rr) of 26 and a periapical image assosiated. After performing the extraction and anatomopathological study the definitive diagnosis is of SOT.
Material and Methods: We make a research in Pubmed with the words 'odontogenic squamous tumor NOT squamous cell carcinoma'.

Results: A total of 216 papers were obtained, excluding all that talks about SOT, and obtaining a total of 49 papers.

Discussion: The SOT is usually presents in relation to dental structures with a very wide age range. It has more prevalence in the mandibule, but we also found described cases in the jaw. The image is generally asymptomatic and may be associated with mobility and sensitivity to percussion. Radiographically, it presents as a unilocular radiolucent area.

Conclusions: It is a rare and benign lesion, but carcinomatous transformation has been described. The definitive diagnosis is anatomopathological. The initial treatment is conservative, although there are more aggressive treatments.

Patient follow-up is essential, but time is not established.

\section{- Poster 51}

TITLE:- Comparative study on the incidence and location of caries in two different age population.

AUTHORS: Martín Carreras Presas C, Barbero Fernández L, Barbero Palao A, Gutiérrez Cárdenas L, Benito Vicente MC.

Facultad de Ciencias Biomédicas. Departamento de Odontología del adulto. Universidad Europea de Madrid.Clinica Dental Dr. Barbero. Práctica Privada. Madrid.

Introduction: Geriatric patients may suffer from drug induced xerostomia and thus an increased risk of tooth decay. Likewise, some of these patients may present difficulties in removing dental plaque, being more susceptible to the progression of the most prevalent oral diseases.

In turn, adolescents, by the diet intake and hygienic habits can be considered at risk for developing caries. Both populations are susceptible to present various oral pathologies.

Objectives: The purpose of this descriptive study is to determine if there are any differences in caries incidence and location, when comparing these two different age groups.

Material and Methods: A literature review was carried out in Pubmed, on oral pathologies and caries in the elderly and adolescents.

30 adolescents and 30 geriatric patients were examined clinically and radiographically, and the number and location of cavities and fillings were noted. 
Results: We found out a higher incidence of caries in the geriatric group compared to the adolescent group. Regarding the localization, the elderly patients had mostly caries on the vestibular surfaces unlike the adolescents who presented them on the occlusal surfaces.

Conclusions: Both populations are more susceptible to develop decays, being the geriatric group at a higher risk.

In addition it was observed that the most common area of caries presentation in the case of the geriatric patients was the labial or buccal surface and in the group of adolescents the occlusal surface.

\section{- Poster 52}

TITLE:- Dental Management of Geriatric Patients With Cardiovascular Disease.

AUTHORS: Govani A, Awan N, Pucci F, Govani A, Martín Carreras Presas C, Somacarrera Pérez ML.

Facultad de Ciencias Biomédicas. Departamento de Odontología del adulto. Universidad Europea de Madrid. Práctica Privada en Centro Dental Manor. Leicester. Reino Unido.

Introduction: Gerodontology is a speciality of dentistry which is dedicated to promoting oral hygiene to the senior patients, preventing and treating their oro-facial problems. We consider patients over the age of 65 years old as "Geriatric". The majority of these patients present with systemic diseases which consequently change our approach to their therapeutic treatment.

Due to the ageing of the population and the consequent increase in the number of medical conditions associated with age, it is the duty of dentists and health professionals to know and identify the special needs of these patients in order to offer them the appropriate therapeutic options as consequence to the medical conditions they present.

Case Report: The following patient is a female aged 93. She is on a variety of medication which includes Salotol to manage her cardiac arrhythmia, and Doxazosin and Co-Diovan for high blood pressure. The last INR recorded was 2.3 prior to treatment. She came to the clinic presenting with a number of problems, which required multiple extractions, a veneer, and a removable denture. The protocols of the dental management of this patient are reviewed and presented.

Conclusions: Understanding the dental management of patients with cardiovascular disease in the dental clinic is very important due to its high prevalence in our environment. The dentist must know the current medical situation of the patient in order to be able to become competent in the way his therapeutic attitude with the different treatments which need to be implemented on the patient.

\section{- Poster 53}

TITLE:- Implant rehabilitation in a systematically compromised patient.

AUTHORS: Garay García AL, Laime Rojas CS, Castañeda Vega PM, López López J, Jané Salas E.

Máster de odontología en pacientes oncológicos e inmunocomprometidos. Facultad de Medicina y Ciencias de la salud, UFR Odontología, Campus Bellvitge, Barcelona España.

Introduction: Large cell lymphoma (LNH) is a type of cancer produced in the lymphatic system, given multiple locations in the lymphatic system. The disease can present any part of our body, also can be associated with other systemic pathologies (ES) Diabetes mellitus (DM), arterial hypertension (HA) and heart diseases (C). LNH may have repercussions in the mouth such as periodontitis, that may present from a discrete gingival inflammation and can progress until the loss of alveolar crest bone, with total destruction of the Periodontal tissues, causing tooth loss and possible infections at a distance, their recognition as a risk factor for health, is of great importance.

Clinical Report: A 78-year-old patient with a medical history of type II diabetes mellitus diagnosed in 1956. Diagnosed hypertension (1987). Cholesterol, cardiac arrhythmias and angina in 1992. Surgically treated largecell lymphoma located in left limb in 2006, periodontitis with grade II mobility diagnosed in 2012. Clinical examination shows that it is lower total and partial upper edentulous. It is rehabilitated with an overdenture with a lower bar, with a fixed upper prosthesis (11-17, 21-22), an implant and a crown are placed in the position of 23 .

Conclusions: Oral rehabilitation in patients with NHL with associated systemic diseases should be considered as possible following the appropriate diagnostic and therapeutic criteria

\section{- Poster 54}

TITLE:- Implantoprotesic rehabilitation in patient with scared carcinoma in lower lip. Clinical case.

AUTHORS: Valero Laprea A., Omaña Cepeda C., Castañeda Vega PM., Lopez Lopez J., Jane Salas E.,

Máster de Odontología en Pacientes Oncológicos e Inmunocomprometidos. Facultad de Medicina y Ciencia de la Salud. UFR de Universitat de Barcelona.

Introduction: The oral squamous cell carcinoma has a high prevalence. It is the most common malignant neoplasm of the oral cavity and the lip is the most frequent localization. Of multifactorial etiology, related to environmental factors, tobacco and alcohol. We present a 
case of an integral rehabilitation in a patient who suffered squamous carcinoma in the lower lip. It describes the procedures and the corresponding treatment of the case.

Clinical Case: We present the case of a patient, a 77year-old man with a cancerous history (OSCC in lower lip). A complete implantoprosthetic rehabilitation was performed, under sequence of extractions (32.33.43.44) and deferred placement of 6 implants in the maxillary (zone 12.13.15.22.23.25) for later rehabilitation through milled - bar implant - supported mandibular overdentures and post insertion controls.

Conclusions: Although the oncological treatment produces large number of complications at the oral cavity level, we can present a case in which the implant-supported and retained treatment is an adequate alternative for the rehabilitation of patients with oncological antecedents in which many structures have been lost. A multidisciplinary team with a correct treatment plan is needed to obtain favorable results in these patients.

\section{- Poster 55}

\section{TITLE:- Esthetic dentistry with prosthetic treatment. case report.}

Dávila Monzón C, Castañeda Martin P, Omaña Cepeda C, Estrugo Devesa A, López López J, Jané Salas E.

Máster de Odontología en Pacientes Oncológicos e Inmunocomprometidos. Facultad de Medicina y Ciencias de la Salud, Odontología. Universidad de Barcelona.

Introduction: The esthetic and functional rehabilitation of patients after oral surgery for excision of head and neck tumors result in a challenge for dentistry practitioners. The execution of prosthodontics treatments must be plan in an individual way, paying attention to conventional aspects and those specifics from the illness, it is related with multiple conditions, among others: type of reparative surgery, the destruction and loss of oral structures and the remnants, the secondary effects of oncological treatments, complementary treatment received, and systemic situation. Additionally, we need to take into account patient's life expectancy, psychological aspects, social and economic situation from each case.

Case Report: We present a 65-year-old woman, diagnosed in 2009 of OSCC in left side of mandibular body, treated with partial resection, from the coronoid process until parasymphysis, unilateral lymph node resection and radiotherapy. She complained of "chewing discomfort" in a fixed dental bridge from 21 to 28 and chronic pain in left side face. We did a prosthetic treatment according to the needs and patient expectations.
Conclusions: When we performance prosthetic rehabilitation in patients with oncological history, we should attempt to give them back, in addition to the function, favorable aesthetics patterns for each case, trying a "restitutio ad integrum" for offer them a better quality of life.

\section{- Poster 56 \\ TITLE:- Osseointegrated implants in a patient with chronic erythematous candidiasis.}

\author{
AUTHORS: Avellaneda Gimeno E, Egido Moreno \\ S, Marí Roig A, Jané Salas E, Roselló Llabrés X, \\ García Ramón S. \\ Máster en Medicina, Cirugía e Implantología Oral. Facultad de Me- \\ dicina y Ciencias de la Salud, Odontología. Universidad de Barce- \\ lona.
}

Introduction: Chronic erythematous candidiasis is an opportunistic, inflammatory superficial infection where clinically it is shown as a reddish area with poorly defined borders on the oral mucosa. The main microorganism involved is Candida albicans. Local and systemic factors facilitate the development of the disease, which is why it is commonly referred to as "the sickness of the sick patient".

The implant-supported prostheses maintain a favorable oral rehabilitation providing occlusal stability to the patients and improving the quality of life of these patients.

Clinical Case: We report the case of a 60-year-old female patient with chronic erythroid candidiasis that is difficult to treat. She carries an oral rehabilitation with mixed prostheses for more than 10 years. The patient does not report pathological antecedents of interest or known drug allergies. She was diagnosed in 1993 presenting multiple episodes of exacerbation with important pain and angular cheilitis and being treated with topical and systemic antifungal agents.

In 2005 , due to the difficulty of controlling the outbreaks, the option of an implant-supported rehabilitation was proposed with the placement of 6 osseointegrated implants in each arch to support a hybrid prosthesis. After placement of the hybrid prostheses, the patient did not present recurrence of candidiasis.

Conclusions: Rehabilitation with fixed implant-supported prostheses should be considered and the possibility that poor teeth may constitute a reservoir for the perpetuation of refractory to treatment - chronic erythematous candidiasis. 


\section{- Poster 57}

TITLE:- The role of leukocyte and platelet rich fibrin (l-prf) in regenerative oral surgery and osseointegration.

AUTHORS: García Riart Monzón M, Paredes Rodríguez VM, González Serrano J, Sáez Alcaide LM, Cortezo Vidal V, Hernández Vallejo G.

Departamento de Estomatología III. Facultad de odontología. Universidad Complutense de Madrid.

Introduction: The recovery of lost oral tissues is a daily challenge for oral surgeon, using techniques known as guided tissue regeneration (GTR) and guided bone regeneration (GBR). These are especially sensitive technique, but with the advent of new developments and surgical protocols is possible to achieve increasingly predictable results. In this context we can place the graft autologous leukocyte- and platelet- rich fibrin (L-PRF), one of the four main families of platelet concentrates for surgical use.

Objectives: The aim of this review is to study the effectiveness of L-PRF in regenerative oral surgery and osseointegration, establish its indications and study possible current protocols.

Material and Methods: We performed an updated literature review in database MEDLINE using the keywords "leukocyte and platelet rich fibrin", and "guided bone regeneration".

Results, Discussion and Conclusions: The L-PRF is a graft easily obtained by a simple procedure of drawing blood without anticoagulant and centrifuged immediately. Is obtained a clot of L-PRF which can be used directly or compressed into a membrane without damaging the cells and growth factors. The architecture of fibrin, unlike other platelet concentrates containing leukocytes, platelets, mesenchymal stem cells and growth factors that promote healing in any surgical wound, including hard and soft tissues. Only it works as a natural biological scaffold or in combination with any type of bone graft. We are seeing the trend of surgical procedures is in favor to natural substances. Today guided bone regeneration naturally possible.

\section{- Poster 58}

\section{TITLE:- Traumatic neuroma. Case report.}

AUTHORS: Marques Granate A, Schiavo Di Flaviano V Moreno Vicente J, Mishra S, Jané Salas E, López López J.

Máster en Medicina, Cirugía e Implantología Oral. Facultad de Medicina y Ciencias de la Salud, Odontología. Universidad de Barcelona.
Introduction: The Traumatic Neuroma (NT) consists of a benign proliferation of nervous tissue, related to an exaggerated response of the regenerative process. It occurs due to a natural or surgical trauma of a peripheral nerve. Clinically it manifests as a firm submucosal nodule, covered by healthy mucosa and is normally painful on palpation. In the oral cavity, the sites most frequently affected are the lip, tongue and plexus of the mental nerve. Histologically it consists of a proliferation of nerve fascicles, including axons, Schwann cells and fibroblasts in a background of collagen.

Case Report: We present the case of a 69-year-old female patient who was referred to the department of oral medicine, surgery and implantology at the University of Barcelona for evaluation of a nodular lesion in the lower right jaw. During examination we palpated a nodular, submucosal lesion at the bottom of the vestibule of 4445 , firm, $1 \mathrm{~mm}$ in size, mobile, painful on palpation and similar in color and texture to the healthy mucosa. The patient had a medical history of glaucoma, osteoporosis and hypercholesterolemia. She is being medicated with injectable Denosumab every 6 months since 2014. With a presumptive diagnosis of mucocele or fibroma, we decided to perform an excisional biopsy of the lesion attached to the submucosa. The histopathological analysis resulted in NT.

Conclusions: Despite its low prevalence, the NT is a type of lesion that must be considered in differential diagnosis of painful nodular lesions, close to anatomical nerve structures such as NDI.

\section{- Poster 59}

TITLE:-Actinic cheilitis: a case report.

AUTHORS: Sances Masero A, Sardañés Martínez S, Rodríguez de Rivera Campillo E, Estrugo Devesa A, Jané Salas E, López López J.

Máster en Medicina, Cirugía e Implantología Oral. Facultad de Medicina y Ciencias de la Salud, Odontología. Universidad de Barcelona.

Introduction: Actinic cheilitis (AC) is a potentially malignant disorder (PMD) that mostly affects the lower lip of men caused by chronic exposure to ultraviolet radiation from the sun. It is estimate that $95 \%$ of lip OSCC originate from chronic ACs.

Case Report: A 75-year-old man who comes to our service to assess ulcerated lesions on the lower lip of several years of evolution. A former construction employee, no toxic habits and with allergy to the penicillin. Has a medical history of cerebral stroke (2012), hypercholesterolemia, hypertension and benign prostatic hyperplasia; it is medically treated with Tromalyt ${ }^{\circledR}$, Simvastatin, Enalapril and Tamsulosin. On clinical examination we 
observe several ulcerated lesions with erythematous and leukoplakia areas in lower lip vermilion. A biopsy was performed to rule out malignancy resulting in: actinic cheilitis. AC is PMD is more common in white men, in the 6th decade of life and predominantly in lower lip vermilion. It is caused by chronic exposure to sunlight or artificial ultraviolet radiation. The clinical presentation is characterized by scaly lips with a dry surface of varied thickness and associated erythema; in more advanced stages appear leukoplakia and/or erythroplakia zones with ulcerations and chronic erosions. The treatment included vermilionectomy, cryosurgery, electrosurgery, topical retinoids, topical application of 5-fluorouracil, photodynamic therapy and vaporization with $\mathrm{CO}_{2}$ or $\mathrm{Nd}: \mathrm{YAG}$ laser.

Conclusions: $\mathrm{AC}$ is a precancerous lesion so it is of great importance an early diagnosis, a correct follow up and treatment of these lesions.

\section{- Poster 60}

TITLE:- Educating older adults towards healthy habits: a challenge for modern dentistry.

AUTHORS: Martínez-Silva C, Villapol-Carpio N, García-Ramón S, Estrugo-Devesa A, Jané-Salas E, Chimenos-Küstner E.

Departamento de Odontoestomatología, Unidad de Medicina Bucal. Universidad de Barcelona.

Introduction: In the exercise of our profession we are committed to treat various disorders. Considering that the disease is not a process that originates as an isolated event, but results from the interaction of multiple factors, is important propose strategies that are conducive to improving the oral health of our elderly patients. Misuse of drugs, multiple physiological changes that occur due to age, stress and inefficient diet, in addition to educational and cultural limitations, can compromise health status.

Objectives: Raise awareness among professionals in contributing to the education of older patients towards healthy living habits.

Material and Methods: Systematic review of the literature. Presentation and discussion of clinical cases, by way of example.

Results and Discussion: Based on the literature consulted, balanced eating patterns are proposed, as well as regular physical activity and eliminating toxic habits. Inference: Is important to complement clinical treatments with education and implementation of healthy living habits, to achieve a better quality of life and decrease the risk of presenting diseases, oral and general.

\section{- Poster 61}

TITLE:- Points in common between the periodontitis and diabetes type 2 . An approximation in the system cell involved and the stress oxidatívo.

AUTHORS: Alvarez Perez JD, Ramos López J, Álvarez Conde $\mathbf{J}$.

Práctica privada y Universidad de Sevilla.

Objectives: Find parameters in common in those two pathologies and the system cell associated.

Material and Methods: NCBI PUBMED central search, with the terms: Oxidative stress and diabetes 2; periodontitis and oxidative stress.

Results: The junctional epithelium at the level of the gingival sulcus and the vascular endothelium of small blood vessels, determined in both disorders an increase in reactive oxygen species that are expressed by an increase of the NADP oxidases.

These oxidases generate damage cell membranes and sensitising to the adjacent fibroblast in the adventitia layer of endothelial vessels.

Another involved cellular system are infiltrating cells, T and $\mathrm{B}$ lymphocytes that appear in front of an aggressive bacterial response or metabolic response (AGEs).

Conclusions: There is an increase of affectation brain in those cases serious where are associated both pathologies.

The cases of diabetes with macro albuminuria intense and periodontitis severe, are associated with greater mortality cardio-renal.

In the periodontitis chronic Decompensated with increase of the stress oxidativo, not is needed that there is plate dental.

E 1 E.O. cell is necessary for the maintenance of the homeostasis in the body for a correct functioning; but when is generates in excess, or is maintained during long time, just being harmful in both pathologies.

\section{- Poster 62}

TITLE:- Oral manifestations of Diabetes Mellitus. Systematic review.

AUTHORS: Estrugo Devesa A, Mauri Obradors E, Palacios Garzón N, Jané Sala E, Viñas Ciordia M, López López J.

Departamento de Odontoloestomatología. Universidad de Barcelona. L'Hospitalet, Barcelona, España.

Background: Diabetes Mellitus has become a global epidemic and presents many complications, usually proportional to the degree and duration of hyperglycemia. 
The aim of this systematic review was to investigate the different oral manifestations associated with Diabetes Mellitus.

Material and Methods: A MEDLINE search for "Diabetes Mellitus and oral manifestations" was performed. Inclusion criteria were as follows: human clinical studies with a minimum of 30 patients; studies published between 1998 and 2016. Nineteen studies fulfilled the inclusion criteria and were analyzed, assessing the strength of scientific evidence according to recommendations made by the Centre for Evidence-Based Medicine, Oxford (OCEBM), which permits adequate assessment of prevalence studies.

Results: A total 3.712 patients (2.084 diabetics) were included in the studies reviewed. Of the 19 studies analyzed, 4 were longitudinal studies and 15 cross-sectional studies. Periodontal disease, periapical lesions, xerostomia and taste disturbance were more prevalent among diabetic patients. An association between diabetes and caries and mucosal lesions proved positive in 5 out of 10 studies.

Conclusions: Despite multiple oral manifestations associated with DM, awareness of the associations between diabetes, oral health, and general health is inadequate. It is necessary for doctors and dentists to be aware of the various oral manifestations of diabetes in order to make an early diagnosis.

\section{- Poster 63}

TITLE:- Oral disorders in patients with Parkinson's disease.

AUTHORS: Moreno Quispe LA, Mansilla Romaní M, Salgado Gonzáles C, García García V, Lópe -Pintor R, Hernández Vallejo G.

Título de Especialista Universitario en Medicina Oral, Facultad de Odontología, Universidad Complutense de Madrid.

Introduction: Parkinson's disease is a progressive and chronic neurodegenerative disease that affects the neurons that produce dopamine, altering motor function. The origin is unknown, however previous studies suggest that it responds to genetic and environmental causes. It affects in Spain 2-3\% of the population over 65 years and there is $15 \%$ of cases before 40 years. It has an incidence of 20 cases per 100,000 inhabitants per year. Therefore, the objective of this review is to know the different oral manifestations in patients with Parkinson's for an adequate dental management.

Material and Methods: A bibliographic search was carried out with keywords "Parkinson's disease and dental management" on January 20, 2017 in the Medline (PubMed) database. Inclusion criteria were original review articles or clinical trials developed in humans. Opinion articles, letters to the editor, technical reports and articles published in a language other than English and in animals were excluded.

Results: 26 scientific articles were obtained in an initial search. 17 were eligible, these were revised and only 7 fulfilled expected criteria. Oral manifestations related to the disease are: xerostomia, drug-associated sialorrhea, dysphagia, orofacial pain, periodontal disease, halitosis, mucosal dehydration, difficulty in speech, swallowing and proprioception that aggravates the patient's quality of life.

Conclusions: It is important for the dentist to interact with the patient's usual drugs and early care for the oral pathology with products that relieve xerostomia and support of their caregivers.

\section{- Poster 64}

TITLE:- Local anaesthetics: clinical relevance and efficacy of the liposomes in oral cavity.

\author{
AUTHORS: Blanco Jauset P, Mishra S, García \\ Ramón S, Estrugo Devesa A, Jané Salas E, López \\ López J. \\ Master en Cirugial, Medicina e Implantología Oral. Universidad de \\ Barcelona.
}

Aim: To determine the efficacy and clinical applicability of the use of local anaesthetics encapsulated into liposomes in dental treatment.

Material and Methods: A literature review of the last 5 years (2011-2016) in the Medline Pubmed database was done using the key words: local anaesthetic and oral liposomes. All the English full-text articles were included.

Results: Good results were obtained in the infiltration of liposomal articaine (multilamellar) in tissues with no signs of inflammation, although the association of $4 \%$ articaine (with adrenaline 1:100.000) is still the best choice in cases of inflammation. A statistically significant increase in post-operative results using liposomal bupivacaine (Exparel) compared to the classic form of bupivacaine in the endodontic treatment was not demonstrated.

Liposomes are phospholipid vesicles used as vehicles for active ingredients which have demonstrated a high cutaneous and percutaneous penetration, providing a slow releasing of the local anaesthetic and contributing to a better superficial anaesthesia. In case of topical anaesthesia in the palatal mucosa, the liposomal form of $5 \%$ lidocaine presents the same in vivo and in vitro characteristics as EMLA, as well as the liposomal form of $1 \%$ ropivacaine. 
Conclusions: It is necessary to develop better topical anaesthetics for use in the palatal mucosa. Further studies are needed to investigate the encapsulation of anaesthetics into liposomes and increasing their efficacy.

\section{- Poster 65}

TITLE:- Fibrous hyperplasia in generalized gingival hyperplasia compatible with antihypertensives intake.

\author{
AUTHORS: Sardañés Martínez S, Sances Masero A, \\ González Navarro B, Jané Salas E, López López J. \\ Máster en Medicina, Cirugía e Implantología Oral. Facultad de Me- \\ dicina y Ciencias de la Salud, Odontología. Universidad de Barcelona.
}

Introduction: Gingival hyperplasia or rather enlargement of the gingiva (AG) is defined as an enlarged gingiva and its etiology is multifactorial. The dental management of this pathology is usually protocolized once the cause is found. However, it is often necessary to do a biopsy to confirm the diagnosis.

Clinical Case: A 71-year-old female patient without allergies and a medical history of hypertension, hypothyroidism, osteoarthritis and Type 2 Diabetes Mellitus presents a generalized gingival hyperplasia in the maxilla and mandible combined with a nodular lesion on the base of the buccal keratinized gingiva in 3.6, with 3 months of evolution. The patient came derived by her usual dentist to study the exophytic lesion. Once the patient was explored, a presumptive diagnosis of pyogenic granuloma was established and an excisional biopsy of the nodule was performed. The histopathological result showed fibrous hyperplasia.

Taking into account the anatomopathological result, we considered as a probable diagnosis a hyperplastic lesion reactive to the combination of the medication and the presence of plaque and calculus. The patient was derived to a tartrectomy and an appointment with her regular doctor for the possible modification of the intake of those drugs. The general practitioner reduced the calcium channel blockers and introduced a diuretic. Gingival enlargement improved in controls every 15 days due to the change of medication.

Conclusions: Gingival hyperplasia should always be treated with long term assessment and from a multifactorial view.

\section{- Poster 66}

\section{TITLE:- Use Of Hyaluronic Acid In Oral Pa- thologies Of The Geriatric Patient.}

\footnotetext{
AUTHORS: Almazán Martínez S, Formini E, Anastasio D, Gómez-Font R, Herranz- Calzada A, David Ubiñas K.
}

Faculty of Biomedical Sciences. Adult's Dentistry Department. European University of Madrid.

Introduction: The oral mucosa performs essential protective functions that affect the general health of the patient. The oral epithelium has been reported to become thinner with age and the tissue regeneration and disease resistance are decreased. The use of dentures, systemic diseases and the use of drugs has been associated with certain pathologies such as oral candidiasis, thrush, angular cheilitis and traumatic ulcers. In addition, the degenerative processes of these patients also affect the TMJ. HA, discovered by Meyer and Palmer in 1934, is a carbohydrate found mainly in the extracellular and pericellular matrix and appears in most connective tissues of living organisms. Some studies support that AH may contribute to the treatment of these pathologies.

Objectives: To assess the potential of HA use in relation to the mentioned pathologies in the elderly patient.

Material and Methods: Some articles between 2002 and 2016 were searched in the PubMed, Medline, and Scholar Google databases with the following keywords: elderly, geriatric, older, oral cavity, disease, hyaluronan.

Results: Multiple studies consider HA as a substance with great curative and reparative potential for its moisturizing, lubricating, viscoelastic, antiadhesive, bacteriostatic, antifungal, anti-inflammatory and mediating properties of cellular processes, as well as being nonreactive, mutagenic, immunogenic and antigenic.

Conclusions: The HA seems to have a promising future in the geriatric dental field due to its beneficial properties and its poor contraindications, although more studies are needed.

\section{- Poster 67}

TITLE:- Prevalence of oral mucosal lesions in institutionalized elderly people attending the Master of Medicine, Surgery and Oral Implantology (University of Barcelona).

AUTHORS: Paulo Rodríguez E, Egido Moreno S, Marques Granate A, Arranz Obispo C, Jané Salas E, López López J.

Máster en Medicina, Cirugía e Implantología Oral. Facultad de Medicina y Ciencias de la Salud, Odontología. Universidad de Barcelona.

Objective: The aim of this study was to determine the prevalence of biopsied oral mucosal lesions in institutionalized elderly people and to compare it with the non-elderly population.

Material and Methods: Observational and retrospec- 
tive study, 374 total oral lesions were analysed, the last two years (January 2015 to January 2017) in the oral medicine, surgery and implantology master of Barcelona University. The study was divided in two age groups: elderly ( $\geq 65$ years of age) and non-elderly ( $<65$ years of age), and clinical series parameters were collected.

Results: 374 lesions were obtained, 121 from elderly people ( $>65$ years) and 253 from non-ancient group. The most prevalence lesions in ancient group were: fibroma $(n=26$, $26.4 \%)$, root cysts $(n=10,8.2 \%)$, lichenoid reaction $(n=18$, $14.8 \%)$, chelitis $(n=4,3.33 \%)$, leukoplakia $(n=24,19.83 \%)$, eritroleukoplakia $(n=7,5.78 \%)$ and COCE $(n=3,2.47 \%)$. Non- ancient results were: fibroma $(n=65,25.69 \%)$, root cysts $(n=56,22.13 \%)$, lichenoid reaction $(n=13,5.13 \%)$, chelitis $(n=2,0.7 \%)$, leukoplakia $(n=28,11.06)$, eritroleukoplakia $(n=2,0.7 \%)$ and COCE $(n=1,0.3 \%)$.

Conclusions: Actually, in Spain, there are few epidemiological studies on oral lesions. Such studies are necessary to take preventive measures, especially the elderly population, the main risk group of premalign and malign oral lesions, reflex in this study with 30\% (>65 years) vs $12.06 \%$ (<65 years).

\section{- Poster 68}

\section{TITLE:- Management of the patient with endo- crine pathology.}

AUTHORS: Pizarro Genoves. A, Torres Moragues. M, Zubillaga Zabala. P, Ribera JM.

Universidad internacional de Catalunya.

Abstract: The endocrine pathology is very prevalent among our patients. Is necessary to consider the preventive measures that will allow us to make the different treatment plans with security and efficacy.

Objectives: To carry out a bibliographic revision of the different types of endocrine pathologies and expose the differences they have with the healthy patients.

Material and Methods: Systematic review about the different endocrine pathologies consulting Medline and Pubmed databases dated from 2003 until 2016, in en-glish and spanish languages, using as keywords: diabetes, -manejo dental, patología endocrina,síntomas, glándulas suprarrenales, hipertiroidismo, sistema endocrino, estado de salud oral, paciente mayor, alteraciones orales y diagnóstico. The dental textbook "Tratamiento odon-tológico en pacientes especiales" was reviewed.

Results: 9 articles about diabetes and 5 articles about adrenal diseases were selected. The establishment of an early diagnosis, the determining of the degree of pathology, the prevention of infections, the control of stress, anxiety and pain, and the valuation of complications were the majority of the recommendations founded in these articles.
Conclusions: The dental management varies between healthy patients and patients with endocrine disease making important the establishment of an specific protocol for the different types of endocrine pathologies.

\section{- Poster 69}

TITLE:- Is leukoplakia a pathology of the elderly people? Clinical study.

\author{
AUTHORS: Pinto AC, Henriques I, Cardoso I, Tran- \\ coso P, Azul A. \\ Clínica Integrada de Medicina Oral, Lisboa, Portugal.
}

Cancer is described in the literature as the most frequent oral mucosal lesion in the geriatric population and many authors consider that most of them arise from potentially malignant lesions (PML), essentially leukoplakia. In order to evaluate the prevalence of leukoplakia, its clinical characteristics and the relationship between the diagnosis age of these lesions and oral cancer, an observational, cross-sectional and retrospective study was conducted, with the analysis of 16236 patient's medical records from a dental clinic in Portugal, followed by a descriptive and inferential statistical analysis. Oral leukoplakia was diagnosed in $0.24 \%$ of the patients ( $n$ $=39$ ), with male preference $(56.4 \%$ male and $43.6 \%$ female) and between 35-84 years old. The frequency of leukoplakia was higher in the age group of 55-64 years (64,1\% in patients $\geq 55$ years old) .

The most frequent location was the tongue (34\%), followed by the floor of the mouth $(20 \%)$ and the palate (14\%). About 53\% of tongue lesions were located on the lateral border and $41.2 \%$ in the ventral surface. In $20.5 \%$ of the cases it had a multifocal location.

In 4 cases with clinical diagnosis of leukoplakia the histological result revealed the existence of carcinoma.

If we accept the claim that oral cancer arises from potentially malignant lesions, essentially leukoplakia, it is necessary to know at what age these lesions appear and how long this transformation process will take. This will define the approach of leucoplakia lesions: is it necessary a more aggressive intervention or can we control these lesions?

\section{- Poster 70}

TITLE:- Extraction of impacted third molars' complications in elderly patients - is subcutaneous emphysema a justification for prophylactic surgery?

AUTHORS: Henriques I, Pinto AC, Cardoso I, Azul A.

Clínica Integrada de Medicina Oral, Lisboa, Portugal. 
There are many recommendations about prophylactic removal of third molars, mainly because the major incidence of complications when the removal is in elderly patients, like pain, swelling, trismus and subcutaneous emphysema, which is frequently associated with impacted third molars.

Aim: To evaluate all cases in literature about subcutaneous emphysema, gender and age of patients and with witch treatments this complication is associated.

Material and Methods: A literature search in the Embase/Medline databases was conducted.The included articles are dated since 1966.After the analysis of the titles/abstracts we excluded irrelevant and duplicated papers,selecting 90. After analysis of the full texts and bibliographies,we added 19.Those with insufficient information, reviews,animal/laboratory studies were excluded,ending with a total of 51 articles.

Results: There are 54cases reported in literature of subcutaneous emphysema in the last 50years, only in 3 cases it occurs in an elderly patient.The most frequent dental treatments associated are: conservative/endodontic/prosthesis treatment( 25 cases, $46 \%, 1$ elderly patient), surgery for extraction of lower third molars(13cases, $4 \%$ ) and for extraction of other teeth(4cases, $7 \%$ )both with highspeed-handpiece, use of profilaxis spray(3cases, $6 \%$ ), use of air syringe ( 3 cases, $6 \%$ ), laser(2cases, $4 \%, 1$ elderly patient) and 4 cases of others (7\%).

Conclusions: Subcutaneous emphysema in dentistry is uncommon and restorative/endodontic/prosthetic treatments are the area in which they are most described.Only 3 cases in elderly patients were found, none of them being associated with third molars extraction. Both AAOMS/ ADA defend prophylactic removal of third molars mainly because of the complications associated with extraction in a later age.In our study subcutaneous-emphysema is a complication clearly more common in younger patients.

\section{- Poster 71}

\section{TITLE:- Oral care in cancer patients treated with radiotherapy and chemotherapy.}

\author{
AUTHORS: Isern Schaefer C. Luelmo Reverte 0. \\ Olmo González B. Gil Manich V. Ribera Uribe M. \\ Universitat Internacional de Catalunya. Barcelona. Departamento \\ de Pacientes Especiales y Gerodontología.
}

Objectives: Review the oral care protocols that cancer patients follow before, during, and after receiving radiotherapy and chemotherapy.

Material and Methods: A bibliographic review was performed in the PubMed and Medline databases in the years between 2010 and 2016. The following keywords were used: cancer, head and neck, radiotherapy, chemotherapy, complications, management, dental.
A compilation of the recommendations given to oncological patients in treatment with radiotherapy and chemotherapy has been made in hospitals in the metropolitan area of Barcelona.

Results: The most frequent complications in cancer patients collected in the literature have been mucositis, dysgeusia, infectious processes and xerostomia associated with loss of glandular function.

Both the dentist and the patient himself play a trascendental role in the prevention of such complications. These can be minimized by dental examinations, specific oral hygiene instructions, diet control, xerostomia treatment, antiseptic rinses and avoiding the use of dental prosthesis.

Conclusions: There is no universally agreed protocol on oral care for oncology patients.

With the aim of avoiding or reducing the side effects of radiotherapy and chemotherapy treatments, it is necessary to create awareness and inform both the patient and the health professional about the importance of adopting preventive measures, as well as new habits and strengthen oral care.

\section{- Poster 72}

\section{TITLE:- Submandibular calcifications: about a case of submandibular lipoma.}

AUTHORS: Ruíz Sáenz PL, Sanz Alonso J, Santos Marino J, Martín Ares M, Merchán Morales S, Martínez González JM.

Hospital Virgen de la Paloma. Madrid.

Introduction: Lipomas are benign, slow-growing tumors that are painless and usually have a pedicled appearance, normal or yellowish coloration, and of uncertain etiology. From an anatomopathological point of view lipomas can be combined with other cellular elements, giving rise to other varieties such as fibrolipoma, angiolipoma, chondrolipoma, etc. The calcification of the lipomas is very rare, reason why before its presence it makes to realize differential diagnoses.

Clinical Case: A 58-year-old male ex-smoker, who underwent magnetic resonance imaging (MRI) for ganglion disorders, revealed a left submandibular mass of about $2 \mathrm{~cm}$ in diameter that had passed unnoticed by the patient. After a few months of evolution (6 months) the patient begins to feel this tumor and noting its growth with even mild localized discomfort. In the external palpation induration of the tumor is observed, sessile that made us think about the possibility of a benign bone tumor in the left submandibular area. Intraorally there is no palpation of the tumor. It was decided to perform mandibular $\mathrm{CT}$ with $3 \mathrm{D}$ reconstruction where it was observed that the tumor had a calcified nucleus, a complete 
capsule and slightly separated from the mandibular body and basal. Anatomopathological analysis shows that the lesion is a lipoma with a calcified nucleus.

Conclusions: The treatment of lipomas in all its variations is exclusively surgical, extirpating it in its totality. Patients do not usually demand treatment until function or aesthetics are compromised.

\section{- Poster 73}

TITLE:- Implants rehabilitation treatment in lingual OSCC treated patient. Case report.

AUTHORS: Ubieto Sánchez J., Castañeda Vega PM., Schemel Suárez M., Omaña Cepeda C., López López J. Jané Salas E.

Máster de Odontología en Pacientes Oncológicos e Inmunocomprometidos. Facultad de Medicina y Ciencias de la Salud. UFR de Odontoestomatología, Campus de Bellvitge.

Introduction: The oral squamous cell carcinoma (OSCC) is the most common malignant neoplasm of the oral cavity. There is a mutation of the keratinocyte DNA that may be spontaneous or produced by physical, chemical and / or microbiological agents. The treatment consists mainly of radiotherapy (Rdt) and surgery, but in advanced cases chemotherapy may be necessary. This multidisciplinary decision will lead to functional and aesthetic consequences in the oral cavity.

Case report: A 76-year-old patient with a medical history of OSCC in the tongue and hypercholesterolemia. She is referred by his family doctor for prosthetic rehabilitation and assessment of remaining teeth. Indicates placement of implants in the lower maxillary, prior to the diagnosis of OSCC. Treated with Rdt (Doses greater than $60 \mathrm{~Gy}$ in the 4th quadrant and less than $40 \mathrm{~Gy}$ in the 3rd quadrant - 38 sessions) and subsequent partial glosectomy in 2012. It is performed in the Hospital Odontologico de Bellvitge, clinical and radiographic evaluation, extractions in the pieces with indication, and it is decided to perform as an alternative rehabilitator for her condition, an overdenture with bar in the lower maxillary and a superior removable partial denture (RPD).

Conclusions: It has been possible to complete rehabilitation of both maxillary taking into account their medical history of OSCC, Rdt and partial glosectomy, and the control of signs and symptoms added such as hyposialia and xerostomia, which in these cases make treatment difficult.

\section{- Poster 74}

TITLE:- Management of intraarticular temporomandibular disorders in patients with rheumatoid arthritis.
AUTHORS: Rigesti Fertonani M, Moreno Quispe LA, Mansilla Romani M, De Pedro Herráez M, López Pintor RM, Hernández Vallejo G.

Título de Especialista en Medicina Oral, Facultad de Odontología, Universidad Complutense de Madrid.

Introduction: Rheumatoid arthritis (RA) is a systemic autoimmune disease characterized by synovial hyperplasia and chronic inflammation that can lead to severe functional limitations in the joints throughout the body. Like all other joints, the temporomandibular joint (TMJ) may be affected in these patients, producing pain and functional limitation, being the first cause of nondental pain in the orofacial region.

Objectives: To describe the clinical signs that can present these patients and their handling in the dental consultation.

Material and Methods: A bibliographic review was performed in Pubmed and Medline databases.

Key words: Rheumatoid arthritis, rheumatologic functional score, temporomandibular joints, temporomandibular disorders.

Results: The most significant orofacial manifestation of rheumatoid arthritis is the anatomical and functional involvement of the temporomandibular joint, which usually presents with bilateral preauricular pain, stiffness and decreased mobility ranges.

Conclusions: Rheumatoid arthritis is an entity with a high incidence of the geriatric population today. At present it presents clinical signs that vary according to the stage in which the pathology is found. Therefore, it is important to take into consideration the fundamental aspects of this pathological entity to improve the quality of life of the geriatric patient.

\section{- Poster 75}

TITLE:- Devices of local antibiotherapy of prolonged release in the treatment of perimplantitis. Systematic review of the literature.

AUTHORS: Jalil Abumalham Ikbal D, Garnier Rodríguez JL, Buesa Bárez JM, Barona Dorado C, Fernández Cáliz F, Martínez González JM.

Máster de Cirugía Bucal e Implantología Hospital Virgen de la Paloma. Madrid.

Introduction: In the last years different devices of local antibiotics of prolonged liberation appeared and demonstrated more effectiveness in the treatment of periimplantitis.

Objectives: To evaluate the efficacy of local antibiotics therapy in the treatment of periimplantitis, compared it with the conventional treatment and determining the 
variability in probing depth, bleeding and periimplant bone height after the treatment with local antibiotic therapy.

Material and Methods: Systematic reviews of articles published in Medline, Embase and Cochrane databases. Randomized clinical trials, human and systematic reviews were established as limit. The key words were: "periimplant treatment" and "periimplant antibiotics".

Results: Among the 197 valid articles 24 were selected and 10 were excluded after apply criteria exclusion. Three studies met the selection and quality criteria. Probing depth, bleeding and periimplant bone height was assessed.

Conclusions: Regarding mechanical curettage, the use of gel antibiotics in the periimplant pocket seems to offer no significant variation.

\section{- Poster 76}

TITLE:- Gingival involvement of oral lichen planus in 263 patients.

AUTHORS: Cardoso I, Henriques I, Pinto AC, Montenegro R, Trancoso P, Azul AM.

CIMO-Department of Oral Surgery and Medicine - Lisbon-Portugal.

Oral lichen planus (OLP) is one of the most common oral mucosa disorders ( $2.7 \%$ in our study population).

Our purpose was to analyze the prevalence and clinical aspects (location, morphology and symptoms) of the gingival lesions in a total of 263 patients with OLP in a retrospective, observational, transversal and comparative study, by analysis of 9595 patient records. Descriptive, inferential statistical analysis (Chi-Square with significance level 5\%) were performed.

Gingival lesions were diagnosed in $37.6 \%$ of cases (80.8\% female and 19.2\% male), ages between 21 and 83 (average 58.9 years). Erosive/erithematous/ulcerative forms (desquamative gingivitis) affected $91.9 \%$ of these patients (34.6\% of all OLP patients), $46.2 \%$ affected both upper and lower gingiva and $71.4 \%$ in a bilateral way. Erosive/erithematous/ulcerative OLP coexisted with other intraoral locations in $91.2 \%$ patients.

The symptoms, if present, varied from mild discomfort to severe oral pain, with the general trend increasing from the white to erosive forms. None of our oral cancer cases was associated with previous gingival OLP lesions.

OLP affects between 1-3\% of occidental population and mostly females (in our study $2.7 \%$ and $73 \%$, respectively). Around $35 \%$ of our patients have gingival involvement. Differential diagnosis with periodontal diseases is important and periodontologists should be familiar with the most common clinical aspects of gingival OLP. Additionally, in our population, $13.2 \%$ of our gingival
OLP patients had no lesions in other intraoral locations, making differential diagnosis and a correct treatment difficult for the general practitioner.

\section{- Poster 77}

TITLE:- Cutaneous fistula from an odontogenic origin. Regarding a specific case.

AUTHORS: Gala Penagos E, Ruiz de Carlos C, Santos Marino J, Rubio Alonso L, Barona Dorado C, Martinez González JM.

Master on Oral Surgery and Implantology. Virgen de la Paloma Hospital, Madrid.

Introduction: A fistula is an opening in the mucosal or skin of a drainage route created by an organism to allow the exit of purulent material to the outside, thus allowing the natural drainage of an abscess. Skin fistulas of an odontogenic origin are documented in the literature. However, they are often underdiagnosed and hence treated incorrectly.

Skin fistulas, same as intraoral fistulas, are produced as a result of an apical necrosis and an suppurating apical periodontitis. Many patients do not show clinical symptoms and thus the fistula is diagnosed much later than it originated.

Clinical Case: Male patient, 67 years old, attends the Oral Surgery and Implantology Department at the Virgen de la Paloma Hospital, Madrid.

The patient exhibits a skin injury on the left genial region, diagnosed as a boil. Clinical intraoral examination showed an indurated area in the submucous tissue related with a residual tooth root. Panoramic radiography revealed a radiotranslucent image on tooth 36 , consistent with a chronic periapical abscess. The residual tooth root was extracted and the fistulous tract removed.

Conclusions: Cutaneous fistula on the maxillomandibular area may have an odontogenic origin.

\section{- Poster 78}

TITLE:- Autoimmune dry mouth in elderly patients: Scintigraphy and salivary gland biopsy role.

AUTHORS: Garnier Rodríguez JL, Puente Fernández S, Buesa Bárez JM, Barona Dorado C, Fernández Cáliz F, Martínez González JM.

Máster de Cirugía Bucal e Implantología .Hospital Virgen de la Paloma, Madrid.

Background: Primary Sjögren' Syndrome (pSS) is an autoimmune systemic chronic disease characteri- 
zed by lymphocytic infiltration of the exocrine glands, which causes dry mucosa, especially mouth and eye's. PSS prevalence in $>70$ years patients is $15 \%$ and for its diagnosis it's used two main tests: salivary glands scintigraphy (gS) and minor salivary glands biopsy (gB). It has been proved new classification criteria, $2016 \mathrm{ACR} /$ EULAR with high value for $\mathrm{gB}$ results.

Objectives: 1) To report affectation patterns of different salivary gland scintigraphy 2) To describe histopathological findings of gB 3) Prevalence of PSS in patients referred with xerostomia .4) To analyze added value of new diagnostic criteria 2016 ACR/EULAR

Material and Methods: Retrospective observational study of $>60$ years patients with xerostomia and/or recurrent mayor salivary glands swelling referred for pSS screening in 2006-2016. Statistic analysis: chi- square and concordance kappa tests.

Results: 102 patients $(87,3 \% \mathrm{M})$, mean age $67,8+6,2$. Recurrent gland swelling 9, 2\%. Altered gS: 50\%: 32,4\% uptake vs $15,7 \%$ washout, and bilateral submandibular location $24 \%$. gB performed $62 \%$ : normal ,51\%; nonspecific alterations, 31,4\%; focal sialoadenitis, $17,6 \%$. PSS diagnosed in 31 (30, 7\%): 12,7\% focal sialoadenitis and $14,3 \%$, non- specific alterations. Non- specific alterations were present in $43,7 \%$ non- pSS patients. Applying new criteria didn't increase pSS prevalence, with 0, 58 kappa index.

Conclusions: 1) $31 \%$ elderly patients had pSS. 2) Half of patients had scintigraphy alterations, especially uptake's and submandibular predominance 3) Focal sialoadenitis was present in $17,6 \% \mathrm{pSS}$ patients and normal $\mathrm{gB}$, in $50 \%$ 4) New criteria didn't increase prevalence. 